\title{
DEVELOPMENT OF OPTICAL COHERENCE TOMOGRAPHY TECHNIQUE FOR CLINICAL DIAGNOSTICS AND MONITORING
}

\author{
by \\ Marjan Razani \\ M.Sc. Ryerson University, Canada, 2012 \\ A dissertation \\ presented to Ryerson University \\ in partial fulfillment of the \\ requirements for the degree of \\ Doctor of Philosophy \\ in the Program of \\ Biomedical Physics
}

Toronto, Ontario, Canada, 2016

(C) Marjan Razani, 2016 


\section{Author's Declaration}

I hereby declare that I am the sole author of this dissertation. This is a true copy of the dissertation, including any required final revisions, as accepted by my examiners.

I authorize Ryerson University to lend this dissertation to other institutions or individuals for the purpose of scholarly research

I further authorize Ryerson University to reproduce this dissertation by photocopying or by other means, in total or in part, at the request of other institutions or individuals for the purpose of scholarly research.

I understand that my dissertation may be made electronically available to the public.

\section{Marjan Razani}




\title{
Abstract
}

\section{Development of Optical Coherence Tomography technique for clinical diagnostics and monitoring}

\author{
Marjan Razani \\ Doctor of Philosophy, Biomedical Physics \\ Ryerson University, 2016
}

This dissertation describes techniques that use Optical Coherence Tomography techniques developed for the detection of shear wave propagation in different phantoms, and the use of such waves to enhance the transport of nanoparticles in tissue equivalent phantoms. In the first study, we explored the potential of measuring shear wave propagation using optical coherence elastography (OCE) in an inhomogeneous phantom and carotid artery samples based on a swept source optical coherence tomography (OCT) system. Shear waves were generated using a piezoelectric transducer transmitting sine-wave bursts of $400 \mu$ s duration, applying acoustic radiation force (ARF) to inhomogeneous phantoms and carotid artery samples, synchronized with a swept-source OCT (SS-OCT) imaging system. The phantoms were composed of gelatin and titanium dioxide whereas the carotid artery samples were embedded in gel. Differential OCT phase maps, measured with and without the ARF, detected the microscopic displacement generated by shear wave propagation in these phantoms and samples of different stiffness. We present the technique for calculating tissue mechanical properties by 
propagating shear waves in inhomogeneous tissue equivalent phantoms and carotid artery samples using the ARF of an ultrasound transducer, and measuring the shear wave speed and its associated properties in the different layers with OCT phase maps. In the second study, we present a technique to image the enhanced particle displacement generated using an acoustic radiation force (ARF) excitation source. A MEMS-VCSEL swept source Optical Coherence Tomography (SS-OCT) system with a center wavelength of $1310 \mathrm{~nm}$, a bandwidth of 100nm, and an A-scan rate of $100 \mathrm{kHz}$ was used to detect gold nanoparticle displacement. ARF was applied after the nanoparticles diffused into a collagen matrix (of different collagen concentrations and for a tissue engineered MCF-7 breast cancer cell construct). Differential OCT speckle variance images with and without the ARF were used to estimate the particle displacement. The images were used to detect the microscopic enhancement of nanoparticle displacement generated by the ARF. Using this OCT imaging technique, the enhanced transport of particles though a collagen gel after using an ARF excitation was imaged and analysed. 


\section{Acknowledgements}

I would like to sincerely thank my supervisor, Dr. Michael C. Kolios for his support and confidence during this research. His excitement and enthusiasm about physics is not only motivating, but also highly contagious. I feel privileged to have worked with such a knowledgeable professional. I would also like to thank my supervisory committee, Dr. Victor Yang, Dr. Yuan Xu and Dr. Raffi Karshafian for their insightful guidance during this research.

I would like to thank Dr.Azhar Zam, Dr. Lauren Wirtzfeld, Vaskar Gnyawali, Yan Wang and Elizabeth Berndl for their help. I am also grateful to the technical support provided by the lab and physics program; in particular, Luke Yaraskavitch, Arthur Worthington, Matthew Forrest and Graham Pearson for their knowledge in technical issues.

I would like to thank my parents, for their support and for always encouraging me to pursue my desires. This work would not have been possible without their support. 


\section{Table of Contents}

Author's Declaration ................................................................................................................. ii

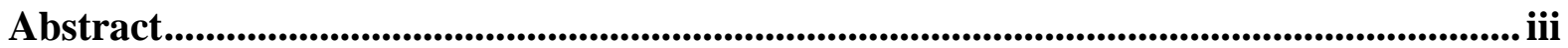

Acknowledgements ............................................................................................................................... v

Table of Contents ...................................................................................................................... vi

List of Tables ................................................................................................................................................ ix

List of Figures......................................................................................................................................... $\mathrm{xi}$

List of Symbols ....................................................................................................................................... xvii

Chapter 1 : Introduction ........................................................................................................................... 1

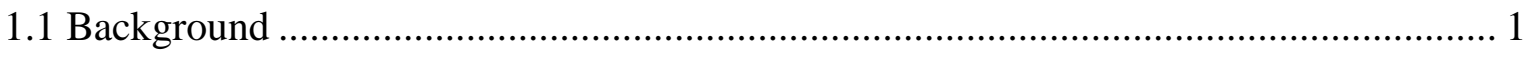

1.2 Basic principles of optical coherence tomography …………………........................... 4

1.3 Motion detection using optical coherence tomography ................................................. 7

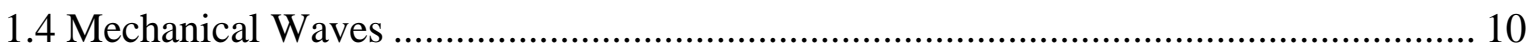

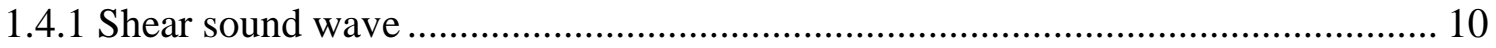

1.4.2 Longitudinal sound waves ........................................................................... 12

1.5 The nature of the shear wave produced with ARF ...................................................... 13

1.6 Imaging modalities used to detect shear wave propagation .......................................... 15

1.7 OCT elastography to detect shear wave propagation and previous work ...................... 18

1.8 Drug delivery and enhanced transport of nanoparticles using ARF ............................ 23

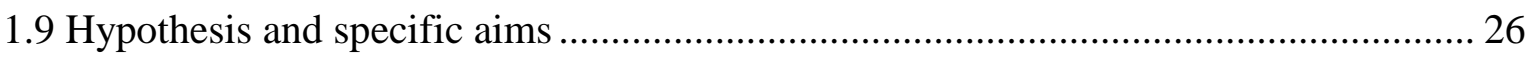


Chapter 2 : Optical coherence tomography detection of shear wave propagation in inhomogeneous tissue equivalent phantoms and ex-vivo carotid artery samples........... 30

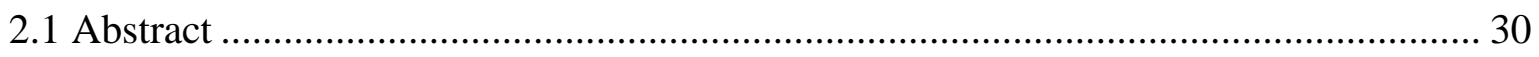

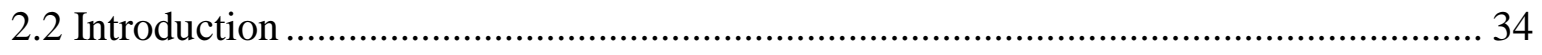

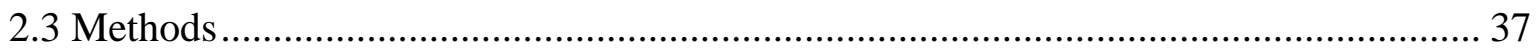

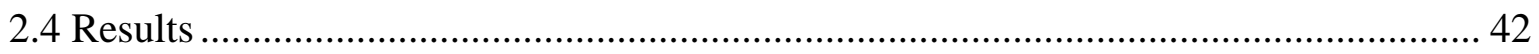

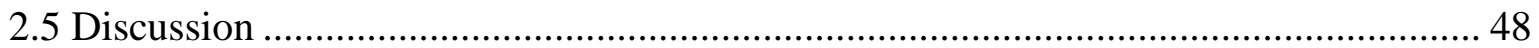

Chapter 3 : Feasibility of Acoustic Radiation Force-Optical Coherence Tomography for Visualization and characterization of enhancement of gold nanoparticles..................... 50

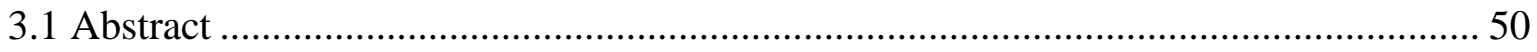

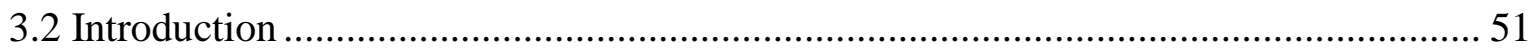

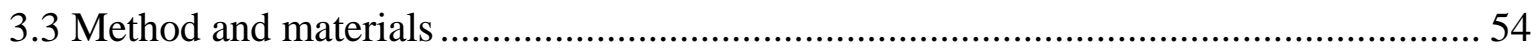

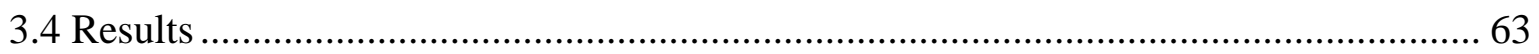

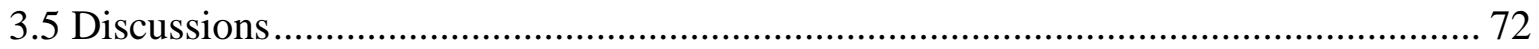

Chapter 4 : Acoustic radiation force enhanced displacement of gold nanoparticles

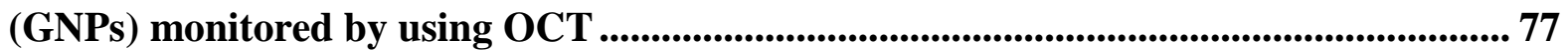

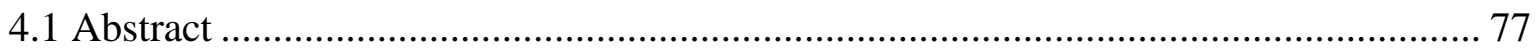

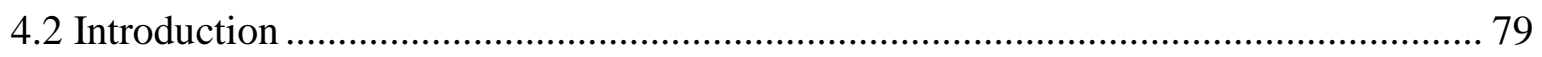

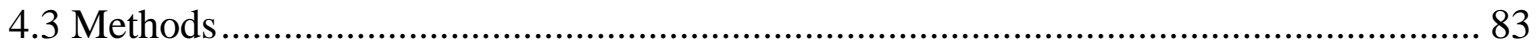

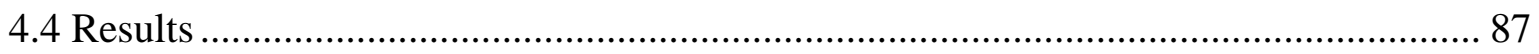


Chapter 5 : Discussion, Conclusions and Future Work ................................................ 102

5.1 Discussion, Conclusions and Future Work ................................................... 102

5.1.1 Measurement of mechanical properties of tissue ........................................ 102

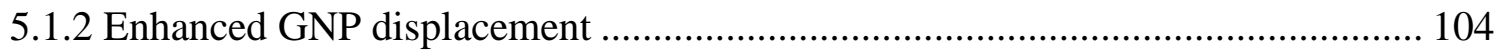

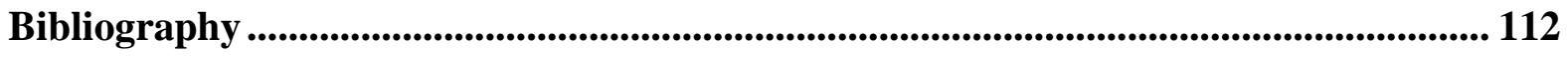

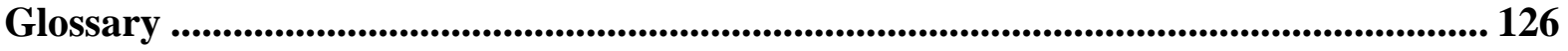




\section{List of Tables}

Table 1-1. Different type of collagens (Adapted from 66) ...............................25

Table 2-1. The mechanical properties of the inhomogeneous phantoms and carotid artery samples (adventitia). The errors for the SW-OCE results represent the standard deviation as

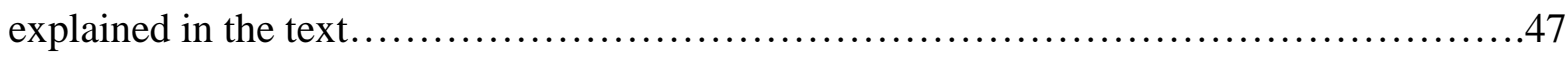

Table 3-1. Parameters which were used in the COMSOL simulation......................63

Table 4-1. Summary of the enhancement of GNPs transport through phantoms of different collagen concentrations $(3 \%, 6 \%, 10 \%[42])$ and the MCF7 module. The ROI(s) are in the vicinity of the focal point of the transducer generating the ARF. Error represents the standard deviation derived from three ROIs selected in the lateral and axial directions. The three ROI(s) for each direction were 50 micron apart to each other. The displacement was calculated for each ROI. From the three ROIs, the average and standard deviation values for each

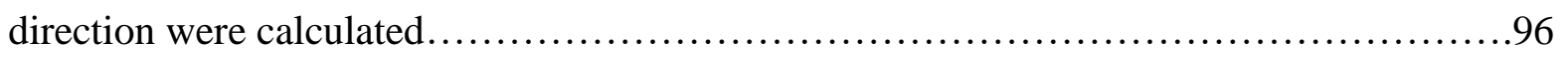

Table 4-2. Summary of the lateral displacements of GNP transport after the application of the ARF in the $3 \%$ collagen phantom (Figure 4 (a)). $X_{0}$ is in the vicinity of the focal point of the transducer which is associated with ROI \# 4 in Figure 4(a). The locations of ROIs: $\mathrm{X}_{0}+$ and $\mathrm{X}_{0}$ - were in the lateral direction in left side and right side of focal point and are associated to the selected lateral ROI(s) of Figure 4(a) ........................................ 98 Table 5-1. Summary of the lateral displacements of GNP transport after the application of the $\mathrm{ARF}$ in the $3 \%$ collagen phantom 
Table 5-2. Summary of the enhancement of GNPs transport through phantoms of different collagen concentrations $(3 \%, 6 \%, 10 \%[42])$ and the MCF7 module. The ROI(s) are in the vicinity of the focal point of the transducer generating the ARF ........................108 


\section{List of Figures}

Figure 1-1 Schematic of a basic OCT setup, based on a Michelson interferometer. The beam splitter can split the light from a low-coherence source into a reference arm and sample arm. Reflections of the sample and reference mirror are recombined into a photodiode.. 4

Figure 1-2 Comparison of the resolution and penetration depth of OCT compared to other imaging modalities that illustrates the trade off between resolution and imaging depth encountered for different medical imaging modalities (resolution elements not to scale). [Adapted from 20]

Figure 1-3 Schematic representation of an obtained speckle variance data set of $\mathrm{N}$ frames and corresponding indices used to label the frame: (i), transverse pixel location (j) and the

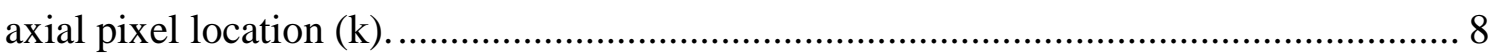

Figure 1-4 Schematic representation of a a) Shear wave and b) Longitudinal wave. The blue arrow shows the particle motion. The red arrow shows the direction of wave propagation. 11

Figure 1-5 Schematic of the principle of the generation of shear wave propagation using a focused ultrasound transducer. In this example, a focused transducer $(20 \mathrm{MHz}, \mathrm{f}-$ number 2.35) is coupled to the sample with gel. A shear wave is generated at focal point of the transducer that is perpendicular to the direction of propagation of the longitudinal wave. 
Figure 1-6 Schematic presentation of shear wave elasticity imaging. Ultrasound can be used to both generate shear waves in tissue, and to image the propagation of these waves either using the same transducer or another transducer. 15

Figure 1-7 A) An ultrasonic probe creates a force that displaces the tissue at the focal point. B) After relaxation of the displaced (pushed) tissue, a low frequency shear wave is generated and the tissue displacement is measured by tracking successive A-lines. C) Generation of plane shear waves using the motion of a supersonic shear source (Adapted from [30]). 17

Figure 1-8 Scanning electron micrographs (SEM) of electrospun collagens type I (A), Collagen type II (B). Collagen type III (C) and collagen type IV (D). SEM scale bars $=1 \mu \mathrm{m}$ (A,B, and C) and $10 \mu \mathrm{m}(\mathrm{D})$. [ Adapted from 77, under the Creative Commons Attribution License (CC BY)]. 25

Figure 2-1 A focused transducer was used to produce an ARF impulse to generate shear waves at the focal point of the transducer. The shear wave propagated in the inhomogeneous tissue equivalent phantom consisted of two layers that were labeled as hard ( $12 \%$ concentration $)$ and soft ( $8 \%$ concentration). 38

Figure 2-2 a) A focused transducer was used to produce an ARF impulse to generate shear waves at the focal point of the transducer, similar to Fig. 2.1. (b) The shear wave propagated in this sample consisted of the carotid artery embedded on top of the gelatin. 
Figure 2-3 A schematic diagram of the ARF-OCE experimental setup. The setup consisted of the existing SS-OCT system, an inhomogeneous titanium dioxide-gelatin phantom, a carotid artery embedded phantom, a focused pushing transducer $(20 \mathrm{MHz}$, f-number 2.35), an amplifier, a 3D micrometer stage and a function generator (Agilent 33250A 80 $\mathrm{MHz}$, Function / Arbitrary Waveform Generator) synchronized with the SS-OCT system. 40

Figure 2-4 a) The B-mode phase map of the phantom was used to measure $\Delta \mathrm{r}$ and $\Delta \varphi$ for the calculation of the shear wave speed. The color scale represents the change of the phase value (radians). The two layers (labeled with the arrows) had different gelatin concentrations. (b) Displacement of shear wave in the inhomogeneous phantom. The color bar represents the particle displacement (in $\mu \mathrm{m}$ ). (c) B-Mode OCT structural image of the inhomogeneous titanium dioxide - gelatin phantom with a shear modulus map (color scale) superimposed on the B-mode image. 42

Figure 2-5 A movie of the shear wave propagation (Media 1) for the tissue equivalent phantom which was created by using different phase offsets (time delays) between the Focusing Ultrasound (FUS) and OCT. The $\mathrm{X}$ axis and $\mathrm{Y}$ axis represent lateral distance(5 $\mathrm{mm})$ and depth(3 $\mathrm{mm})$ respectively. The color scale represent the phase, as in Fig. 2.4(a) $(\mathrm{rad})$.

Figure 2-6 B-mode OCT structural images (a) and the corresponding B-mode phase map (b) of the carotid artery samples were obtained with the SS-OCT system. The color scale 
represents the change of the phase value (radians). (c) The displacement of the sample

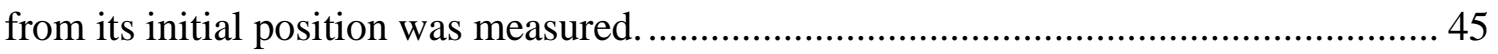

Figure 2-7 Masson trichrome stain histology image of one of the ex-vivo carotid artery samples. 45

Figure 2-8 Mean background phase noise $(\Delta \varphi)$ at four different SNR regions. 48

Figure 3-1 a) Schematic of the experiment for the tubing with a single opening $(100-150 \mu \mathrm{m})$ to allow for the diffusion of the GNPs out of the vessel, b) schematic of the experiment using the porous membrane ( $3 \mu \mathrm{m}$ pores). c) TEM image of GNPs d) TEM image of collagen matrix (scale bar is $100 \mathrm{~nm}$ ). e) Using an impulse generated by a focussed transducer, shear waves can be produced at the focal point that propagate radially outwards. The transducer focal depth for this study was $20 \mathrm{~mm}$. The shear wave generated travels through the collagen matrix also contributed to the displacement of the GNPs which diffused through the collagen. B-mode and SV OCT images were acquired at the focal point for SV analysis 58

Figure 3-2 Processing steps of the algorithm for the measurement of the enhanced GNP delivery. The speckle variance images show the distribution of the GNPs in the collagen matrix. The speckle variance images with the transducer on (which was on for 5 minutes) and then switched off were thresholded and binarized, and then subtracted to show the enhanced GNPs displacement in the axial and lateral direction. The differential speckle variance image (marked in red color in the speckle variance images) was then overlaid with the speckle variance image with the transducer off. The red color pattern 
shows the differential speckle variance image when the ultrasound transducer is ON (US ON) and OFF (US OFF).

Figure 3-3 a) 3D OCT image of tubing after diffusion of GNPs though the opening b) Bmode OCT image of tubing which was embedded into the gelatin phantom and the nanoparticle distribution after the diffusion of the nanoparticles through the opening in the phantom (Media 1).

Figure 3-4 Speckle variance images of the phantom were obtained (a) when transducer was $\mathrm{OFF}(\mathrm{t}=0)-\mathrm{OFF}(\mathrm{t}=10 \mathrm{~s})$.The pattern in the images is representative of the complex spatial distribution of the GNPs that is the result of the transport of the GNPs through the media and the complex interaction of the GNPs with the gel matrix. Speckle variance images were taken when ARF was off at $\mathrm{t}=0$ and at $\mathrm{t}=10$ seconds (time interval was used to be consistent with the methodology used when the transducer was turned on). These images were then analyzed using the differential speckle variance technique and GNPs displacement was calculated in both axial and lateral directions. There were no changes in the location of the peaks of the speckle variance images observed when the transducer was off. (b) Another negative control after 10 seconds was examined to show that the displacement measured solely comes from the ARF when transducer $\mathrm{ON}(\mathrm{t}=0)-$

$\mathrm{OFF}(\mathrm{t}=10 \mathrm{~s})$. These are negative control for the enhancement analysis. 68

Figure 3-5 (a): 100 BM-scans OCT were acquired from GNPs through the collagen. The color map represents the standard deviation value (media2) (b): Speckle variance of after diffusion of GNPs through the collagen, and the calculation of enhanced delivery of the 
GNPs. The red and blue arrows denote the direction of the enhanced transport. The image (a) and (b) were acquired at different locations and time.

Figure 3-6 ARF-induced displacement in phantom. The plots depict the displacement of the phantom mesh at various points in time as a result of ARF excitation and the color bar represents displacement in microns. The displacement fields were taken at times $0 \mathrm{~ms}$, $1.7 \mathrm{~ms}$ (a), $2.8 \mathrm{~ms}$ (b), $3.8 \mathrm{~ms}$ (c), and $4.8 \mathrm{~ms}$ (d) respectively. 71

Figure 3-7 Simulated displacement of two points in the phantom which was induced using the ARF from a $20 \mathrm{MHz}$ transducer, within an isotropic, linear elastic solid and points of a,b,c and d depict to the displacement point at time $1.7 \mathrm{~ms} \mathrm{(a),} 2.8 \mathrm{~ms}(\mathrm{~b}), 3.8 \mathrm{~ms} \mathrm{(c),}$ and $4.7 \mathrm{~ms}$ (d) respectively. The black line represents the displacement in the vicinity of the focal point (A) and red line represents the displacement above this point (B) (see figure3. 6). The two points are $250 \mu \mathrm{m}$ apart. 72 


\section{List of Symbols}

$\begin{array}{ll}\mathrm{P} & \text { Density of phantom }\left(\mathrm{kg} / \mathrm{m}^{3}\right) \\ \mathrm{L}_{\mathrm{C}} & \text { Coherence Length } \\ C & \text { Speed of sound }(\mathrm{m} / \mathrm{s}) \\ C_{S} & \text { Shear wave speed }(\mathrm{m} / \mathrm{s}) \\ \lambda & \text { Wavelength }(\mathrm{nm}) \\ \mathrm{Q} & \text { Quadrature signal } \\ \mathrm{I} & \text { In -phase signal } \\ \mathrm{K} & \text { Bulk modulus (pa) } \\ \mu & \text { Shear modulus }(\mathrm{pa}) \\ \mathrm{E} & \text { Young's modulus }(\mathrm{pa}) \\ \Delta \varphi & \text { phase shift (rad) } \\ \mathrm{F} & \text { Acoustic radiation force }\left(\mathrm{kg} /\left(\mathrm{s}^{2} \mathrm{~cm}^{2}\right)\right) \\ \mathrm{I} & \left.\text { Intensity (W/cm }{ }^{2}\right) \\ \alpha & \text { Absorption coefficient }(\mathrm{Np} / \mathrm{m})\end{array}$

xvii 


\section{Chapter 1: Introduction}

\subsection{Background}

Diagnostic imaging modalities provide images of the physical characteristics of tissue to aid in the detection, diagnosis and monitoring of disease states. Different imaging modalities have been shown to measure tissue displacements and estimate the resulting tissue mechanical properties such as ultrasound or magnetic resonance (MR) imaging [1,2].

Inducing small displacements or perturbations in tissue has several important uses in medicine. First, it could be a useful technique to examine tissue properties in medical imaging. Second, it can be used to enhance the delivery of therapeutic payloads. It is possible to estimate the mechanical properties of tissues by determining displacements that occur in response to an applied mechanical stimulus. There are currently several imaging modalities that can be used to measure tissue displacements and can thus estimate tissue mechanical properties in this manner. One primary such technique that can be used diagnostically, is elastography. It is a technique in which stiffness or strain images of soft tissue are generated [3]. When an imaging modality is employed to image this tissue deformation behavior under a static or dynamic load, the generated image is known as an elastogram. Elastograms contain information about local variations of the stiffness inside a region of interest. This may provide additional clinical information to help in the identification of suspicious lesions, the diagnosis of different disease states and the monitoring of the effectiveness of treatments. 
Other imaging modalities that can be applied to measure tissue displacements and estimate the resulting tissue mechanical properties include ultrasound or magnetic resonance (MR) imaging $[4,5]$. These have certain drawbacks. The drawbacks of MRI and ultrasound are that they do not have sufficient resolution to detect small and subtle elastic variations in tissue such as in small tumors and atherosclerotic plaques [6]. Another modality that can be applied in this manner is Optical Coherence Tomography (OCT) [7]. OCT is an optical tomographic imaging method that has some similarities with ultrasound imaging even though it involves using light. When applied to elastography it is known as Optical Coherence Elastography (OCE). The technology is also flexible and OCT systems can be built relatively inexpensively. OCE measures tissue displacement using OCT and benefits from the high resolution of this imaging technology. OCE is a relatively new elastography technology used to measure biomechanical properties of soft tissue [8,9]. In this thesis, the acoustic radiation force generated by focussed ultrasound has been used to generate displacements in samples and OCT was used to measure the sample mechanical properties.

Displacements that have been generated by the ultrasound have been also applied for the purpose of drug transport enhancement. For example, ultrasound has been shown to enhance the delivery of therapeutic payloads to target tissues [10-13]. Physical parameters of the ultrasound beam (frequency, intensity and pulse duration) can be modified to introduce different mechanisms to achieve this effect $[14,15]$. One of the most effective approaches is to use the acoustic radiation force of a focused ultrasound beam to achieve such delivery [15]. Researchers have shown that such focused ultrasound exposures can be used to enhance 
the delivery of a variety of therapeutically important agents for the treatment of tumors and other diseases [12]. However, it is difficult to assess the efficacy of the ultrasound enhanced delivery in a real-time and non-invasive manner.

Various techniques have been used to monitor the spatial distribution of the nanoparticles (NPs) non-invasively. Two-Photon Luminescence, Confocal Microscopy [16,17] and Optical Coherence Tomography [18]are several imaging techniques that have been used to detect NPs in tissue.

Different imaging modalities such as ultrasound (US) imaging and MRI can be used to monitor the extravasation of nanoparticles from leaky vessels and enhanced delivery of gold nanoparticles (GNPs) [12,19]. Both US and MRI have spatial resolutions in the order of 0.1-1 mm, which is insufficient for detecting small and subtle changes in the spatial distribution of the GNP concentration, such as those that would be occurring in small tumors. However, the superior spatial and temporal resolution of OCT, together with the enhanced optical contrast of GNP, may allow for the detection of small changes in the spatial distribution of GNPs.

This chapter provides an introduction the principles of Optical Coherence Tomography $(\mathrm{OCT})$, the nature of shear wave propagation in tissue, and the enhancement of nanoparticle delivery. This dissertation is devoted to the development of Optical Coherence Tomography techniques to monitor shear wave propagation in tissues and ARF assisted nanoparticle delivery. 


\subsection{Basic principles of optical coherence tomography}

Optical Coherence Tomography (OCT) is an optical tomographic imaging technique that shares many similarities to ultrasound imaging but uses light rather than sound waves to generate images.

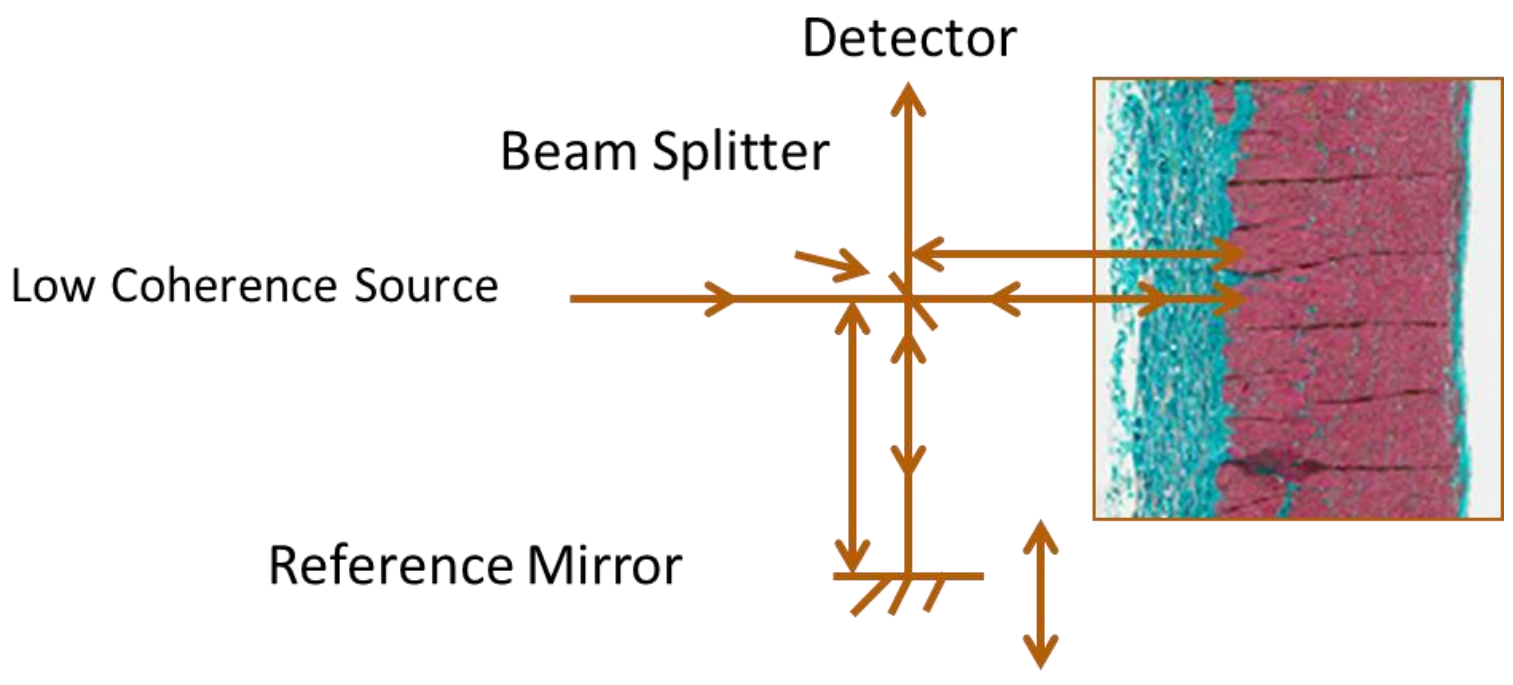

Figure 1-1 Schematic of a basic OCT setup, based on a Michelson interferometer. The beam splitter can split the light from a low-coherence source into a reference arm and sample arm. Reflections of the sample and reference mirror are recombined into a photodiode.

There are several different configurations for setting up an OCT system, most fundamental and common established setup for an OCT system is based on the Michelson interferometer. The Michelson interferometer takes as an input a beam of light (laser) from a source, which is divided into two elements at a beam splitter. Fig 1.1 shows an OCT system in its simplest form: a Michelson interferometer in which the beam splitter divides the beam into two equal arms. Part of the light is directed towards the reference arm and a mirror reflects 
the light back into the detector, and part of the light is directed to the sample arm where a small fraction is backscattered depending on the local sample reflectivity, so that the light interferes at the detector [20]. However, the broadband light causes interference of the optical fields to occur only when the path lengths of the reference and the sample arm are matched to within the coherence length of the light. This interference signal carries information about the sample reflectivity at a depth determined by the reference path length. The axial resolution is one of the most important parameters in OCT systems since it determines how fine the structures can be resolved in the depth direction. The lateral resolution depends on the numerical aperture of the sampling lens. The axial resolution, which is determined by the round tripcoherence length, is given by:

$$
L_{c}=0.44 \frac{\lambda_{0}}{\Delta \lambda}
$$

where $\Delta \lambda$ is the full width at half maximum of the autocorrelation function of the power spectrum and $\lambda$ is the source center wavelength $[21,22]$.

Optical coherence elastography (OCE) measures tissue displacement using OCT and uses images acquired using the high resolution of OCT to make a high-resolution stiffness maps. In this work, we explored the potential of measuring shear wave propagation using an OCT as an imaging modality to measure tissue mechanical properties. Optical Coherence Tomography (OCT) produces images with near histological resolution, which allows for the identification of micron sized morphological tissue structures. Figure 1-2 shows the resolution and penetration depth of OCT as compared to other imaging modalities. 


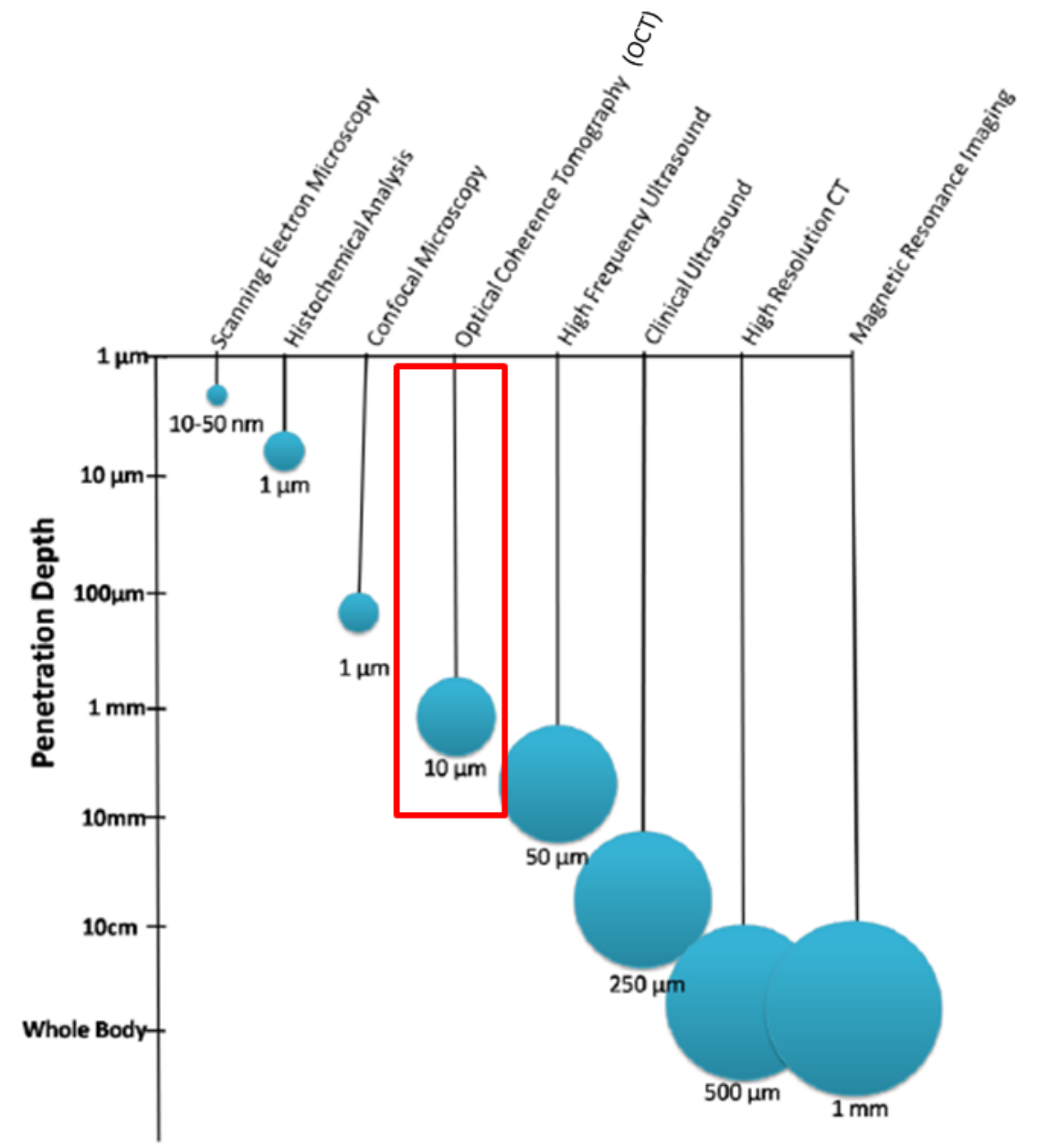

Figure 1-2 Comparison of the resolution and penetration depth of OCT compared to other imaging modalities that illustrates the trade off between resolution and imaging depth encountered for different medical imaging modalities (resolution elements not to scale). [Adapted from 20]

Figure 1.2 demonstrates that the benefit of using OCT is high spatial resolution (10$14 \mu \mathrm{m})$ but the limitation of using OCT is the limited penetration depth $(2-3 \mathrm{~mm})$. Various research groups are trying to make OCT probes to increase the imaging penetration depth [23-25]. 


\subsection{Motion detection using optical coherence tomography}

There are several different techniques that can be used to detect motion using the backscattered data from OCT. One of the techniques is termed Speckle Variance. Speckle variance OCT imaging (SV-OCT) has been shown to be sensitive to the microscopic motion of sub-resolution scatterers. It is a decorrelation measurement method that enables visualization of slow movement independent of Doppler angle which was applied to high frequency ultrasound systems and OCT flow imaging [26].

The algorithm for calculating speckle variance images for both OCT and high frequency ultrasound (HFUS), in its most basic form, requires calculating the variance of pixels from a set of $N$ B-mode images ( $\mathrm{N}$ is referred to as the gate length), acquired from the same spatial location using the equation:

$$
S V_{i j k}=\frac{1}{N} \sum_{i=1}^{N}\left(I_{i j k}-I_{\text {mean }}\right)^{2}
$$

where $\mathrm{N}$ is the number of the $\mathrm{B}$-frames and $\mathrm{j}$ and $\mathrm{k}$ are axial and transverse pixels in the image. SV-OCT has a few of advantages compare to traditional Doppler OCT-based imaging and fluorescence confocal microscopy: (1) Doppler angle Independence (2) High sensitivity (capability of visualizing Brownian motion of highly scattering sources) (3) No contrast agents are typically needed [20]. 


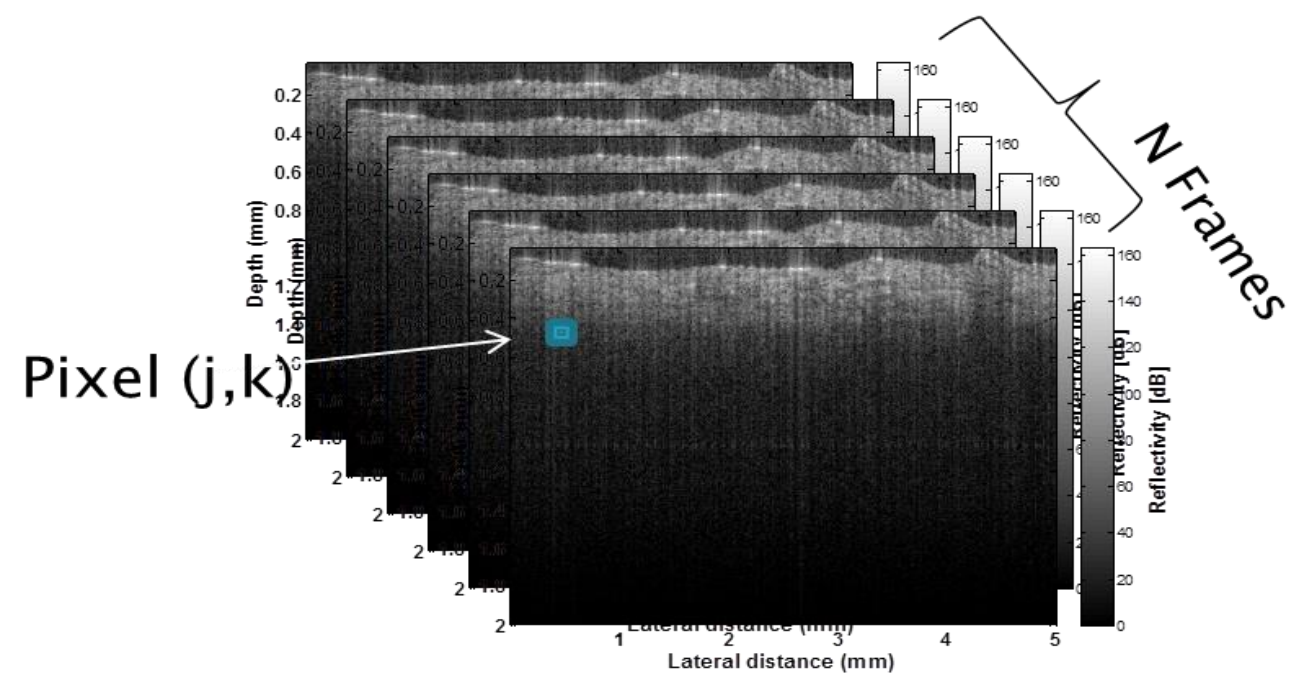

Figure 1-3 Schematic representation of an obtained speckle variance data set of $\mathrm{N}$ frames and corresponding indices used to label the frame: (i), transverse pixel location (j) and the axial pixel location (k).

These characteristics make SV-OCT an attractive tool for detecting Brownian motion and measuring the displacement of unresolved particles based on changes in the speckle intensity. The use of a speckle variance method to measure motion instead of a phase sensitive approach (explained in the next paragraph) allows for the detection of displacements independent of the phase stability of the system.

Another technique for measuring motion is based on phase maps generated by processing the OCT signals. The backscattered OCT signals can be thought to have two frequency elements: the phase modulated carrier frequency and the phase change introduced by the motion of the scattering object. Quadrature demodulation techniques can be used to de- 
tect the phase change introduced by motion. In phase (I) and quadrature (Q) demodulation are used extensively in signal processing and communications. By multiplying the incoming signal by a reference signal, and a reference signal shifted in phase by $90^{\circ}$, two orthogonal vectors are produced. These two vectors create a real and imaginary component of the incoming signal which are referred to as the in-phase and quadrature components of the incoming signal, respectively.

Signals that are captured using this method are compared to a reference wave and separated into in-phase and quadrature components which are represented by:

$$
\mathrm{S}=\mathrm{I}+\mathrm{j} \mathrm{Q}
$$

By using these two vectors, the phase can be calculated using a four-quadrant arctangent function where,

$$
\varphi=\operatorname{Arctang}(\mathrm{Q} / \mathrm{I})
$$

where $\mathrm{Q}$ is imaginary part of the OCT complex OCT signal (OCT_complex), and I is real part of the complex OCT signal (OCT_real). The phase can take values within the range of $\pi$ to $-\pi$. In Chapter 2, we used the phase shift, $\Delta \varphi=\varphi_{2-} \varphi_{1}$, where $\varphi_{2}$ is the phase when the transducer is on and $\varphi_{1}$ is the phase when is transducer is off [27] to detect propagating shear waves. The phase shift between two successive locations, was calculated and then related to motion within the tissue equivalent phantoms.

The accuracy and precision of the sub resolution measurement methods of the OCT system has been discussed in section 5.1.2.4. 


\subsection{Mechanical Waves}

Mechanical waves transfer energy from one location to another location. Two types of mechanical waves of interest to this work are shear sound waves and longitudinal sound waves, which are described below.

\subsubsection{Shear sound wave}

For shear waves, the particle motion is perpendicular to direction of propagation of the bulk wave. Shear wave propagation speed is given by the equation:

$$
C_{s}=\sqrt{\frac{\mu}{\rho}} .
$$

where $\mu$ is shear modulus and $\rho$ is density of medium. For soft tissues the shear wave speed is $\approx 1-10 \mathrm{~m} / \mathrm{s}[28]$.

The speed of shear waves that propagate in soft tissues is directly related to the shear modulus $(\mu)$ of the material (Equation 1.5) $[29,30]$. The shear modulus for soft biological tissues actually spans a much larger range compared to the bulk modulus by several orders of magnitude [31]. The use of the shear modulus as a cancer biomarker has been recently demonstrated using ultrasonic techniques [32].

Shear waves cannot propagate in liquids. However, because most biological tissues have an elastic component, they can support shear wave propagation. Shear waves can be produced by inducing an ultrasonic sinusoidal excitation that displaces bulk tissue along the 
direction of the excitation (the longitudinal wave of the ultrasound beam) which creates a shearing force in the tissue that launches the shear wave.

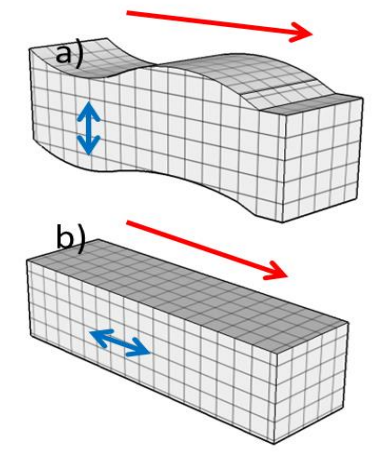

Figure 1-4 Schematic representation of a a) Shear wave and b) Longitudinal wave. The blue arrow shows the particle motion. The red arrow shows the direction of wave propagation.

To recover the tissue shear modulus, two general categories of reconstruction methods have been used: 1) forward methods that minimize the difference between displacements calculated theoretically using models with assumed material properties and the displacements measured experimentally with an imaging device and 2) direct inversion of the differential equation of motion. These methods include the inversion of the Helmholtz Equation, which characterizes the shear wave propagation $[33,34]$, using algorithms that measure lateral time for peak-to-peak displacements [38], tracking the displacement field jitters that are associated with shear waves $[35,36]$ and using a variety of correlation-based algorithms $[37,38]$.

Experimentally, the displacements due to shear waves at each tracking location can be calculated based on a speckle tracking algorithm based on the OCT phase maps generated. The shear wave speed could be calculated using $\Delta \varphi$ and $\Delta r$ calculated from the measured 
phase shift and the distance between the two tracking points. The shear wave speed can then be calculated using the following equation:

$$
C_{S}(\omega)=\frac{\omega \Delta r}{\Delta \varphi}
$$

where $\omega=2 \pi f, \Delta \varphi$ is the phase shift and $\Delta r$ is the distance between the two tracking locations[9]. The shear wave frequency (f) is dependent on several factors, the most dominant of which is the beam width of the excitation transducer.

\subsubsection{Longitudinal sound waves}

For longitudinal sound waves, the particle motion is parallel to the direction of propagation of the bulk wave. For such compressional waves, the speed is given by the equation:

$$
\begin{gathered}
C_{P}=\sqrt{\frac{\lambda+2 \mu}{\rho}} \approx \sqrt{\frac{\lambda}{\rho}}\left(\approx 1500 \frac{m}{s}\right) \\
\text { where } \lambda=K+4 / 3^{\mu}
\end{gathered}
$$

In equation, $1.8 \mathrm{~K}$ is bulk modulus and $\mu$ is shear modulus. For most soft tissues, the longitudinal speed of sound is $\sim 1500 \mathrm{~m} / \mathrm{s}$. The acoustic radiation force is applied in the direction of the longitudinal wave propagation. Moreover, the speed of longitudinal waves is higher than the speed of the typical ultrasound shear waves used in biomedical applications. The acoustic radiation force (ARF) of ultrasound propagating waves has been used to generate such mechanical excitations noninvasively. Traditional compression wave imaging meth- 
odologies, such as US, provide measurements based on the tissue bulk modulus, which is confined to a relatively small range of values for soft tissues compared to the tissue shear modulus.

\subsection{The nature of the shear wave produced with ARF}

The Acoustic Radiation Force (ARF) is produced by a change in the energy density of the incident acoustic field $[39,40]$. The acoustic radiation force is generated by the transfer of momentum from the acoustic wave to the tissue. This can be achieved by using focused ultrasound. The force is applied in the direction of the longitudinal wave propagation and the magnitude of the force can be approximated by:

$$
F=\frac{2 \alpha I}{C}
$$

where $\mathrm{F}\left[\mathrm{kg} /\left(\mathrm{s}^{2} \mathrm{~cm}^{2}\right)\right]$ is the acoustic radiation force, $\mathrm{C}(\mathrm{m} / \mathrm{s})$ is the speed of sound in the medium, $\alpha(\mathrm{Np} / \mathrm{m})$ is the absorption coefficient of the medium and $\mathrm{I}\left(\mathrm{W} / \mathrm{cm}^{2}\right)$ is the temporal average intensity at a given spatial location [41]. When the acoustic radiation force is applied to a given spatial volume for a short time duration, shear waves are produced that propagate away from the focal spot region [42]. Shear-wave propagation speed and attenuation are directly related to the mechanical properties of the tissue. Typical values for the shear wave attenuation coefficient of soft tissues are two or three orders of magnitude higher than that of the attenuation coefficient of compressional waves. Due to the high attenuation of shear waves, shear waves propagate within a very limited area of tissue $[39,43]$. The spatial frequency content of the shear wave will be determined primarily by the width of the ultrasound 
beam (and not the time duration of the excitation unless the excitation duration approaches the natural time constants of soft tissues). This is turn dictates the frequency of the shear wave produced; smaller ARF beams produce higher frequency shear waves. In this thesis the transducer used to produce the ARF had a beam diameter of $245 \mu \mathrm{m}$ (the depth of field was $2.94 \mathrm{~mm}$ and a $20 \mathrm{MHz}$ sine-wave burst of $400 \mu$ s was applied).

Figure 1.5 shows the principle of shear wave generation. Shear waves can be generated by using a focused impulse generated by an ultrasound transducer. The impulse creates a displacement in the direction of ultrasonic beam propagation which is largest at the transducer focus. After the impulse, the material relaxes back to its original state producing a shear wave. The shear wave propagates in the direction perpendicular to the direction of the focused ultrasound propagation.

Titanium dioxide gelatin phantom

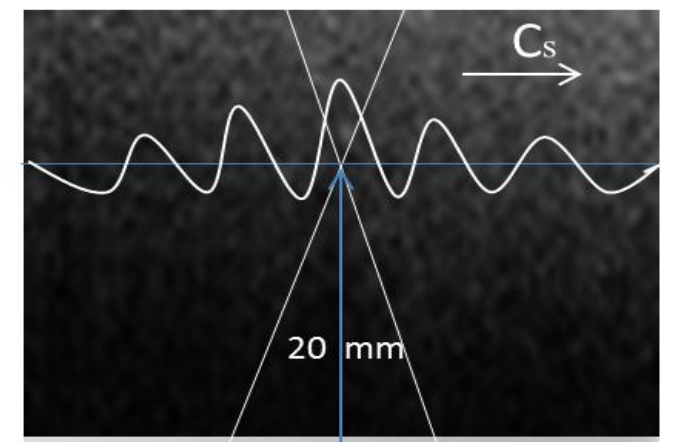

\section{Couplant Gel \\ Focusing Transducer}

Figure 1-5 Schematic of the principle of the generation of shear wave propagation using a focused ultrasound transducer. In this example, a focused transducer (20 MHz, f-number 2.35) is coupled to the sample with gel. A shear wave is generated at focal point of the transducer that is perpendicular to the direction of propagation of the longitudinal wave. 


\subsection{Imaging modalities used to detect shear wave propagation}

There are several imaging modalities that have been used to detect shear wave propagation in tissue: 1) ultrasound (and a variant of ultrasound imaging called supersonic shear wave imaging) 2) MRI and 3) Optical Coherence Tomography.

Ultrasound can be used to measure bulk tissue displacement using algorithms that detect tissue motion. Shear waves can be created in a medium by using a focused ultrasound transducer (such as an ultrasonic phased array or a single focused transducer) and then the tissue motion can be detected by using either the same transducer or other independent ultrasound pulse-echo imaging transducers [44]. In this method for tracking the displacements generated by the ARF excitation source, a reference ultrasound A-line pulse is initially fired for tracking, followed by a high-intensity pulse used to generate the ARF, followed by a series of ultrasound A-line tracking pulses to monitor the resulting tissue displacement.

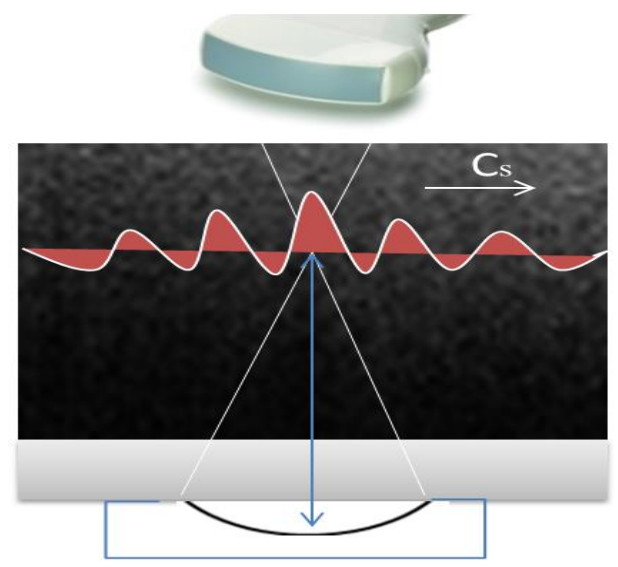

Figure 1-6 Schematic presentation of shear wave elasticity imaging. Ultrasound can be used to both generate shear waves in tissue, and to image the propagation of these waves either using the same transducer or another transducer. 
The second method, a variant of shear wave imaging called supersonic shear wave imaging, involves creating a shear source moving through the medium at a supersonic speed (greater than speed of the shear wave in tissue). This is achieved by successively focusing the ultrasonic "pushing" ARF beam at different depths, where the focus is modified at a speed which is greater than the tissue shear wave speed. Figure 1.7 shows the principle of supersonic shear imaging. Supersonic shear wave imaging can provide the quantitative shear modulus mapping of an entire organ in less than $30 \mathrm{~ms}$ [44]. 
Pushing beam

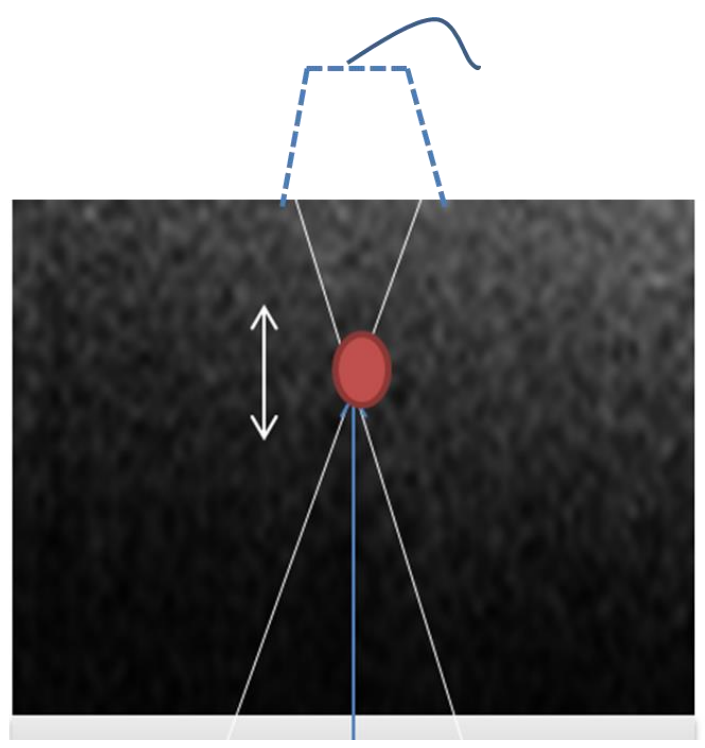

A
Imaging shear wave

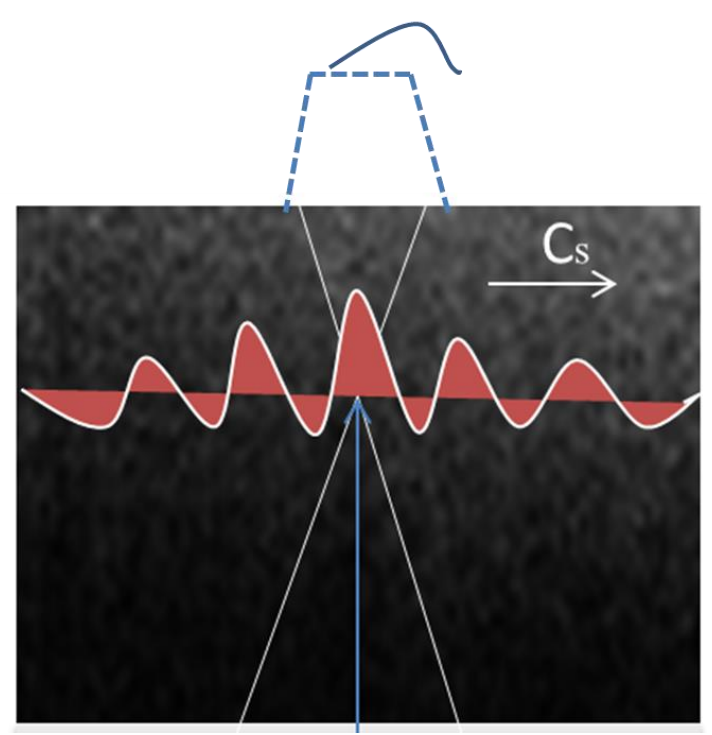

B

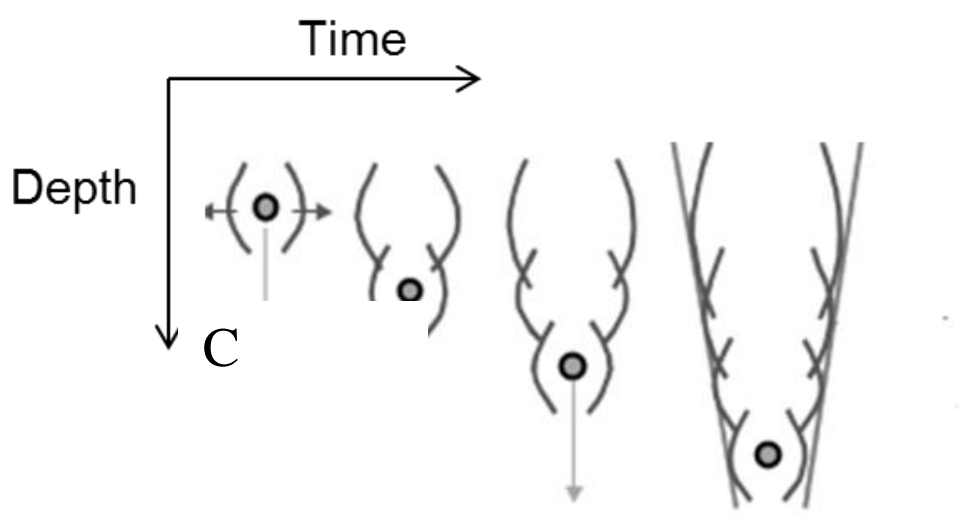

Plane shear waves

Figure 1-7 A) An ultrasonic probe creates a force that displaces the tissue at the focal point. B) After relaxation of the displaced (pushed) tissue, a low frequency shear wave is generated and the tissue displacement is measured by tracking successive A-lines. C) Generation of plane shear waves using the motion of a supersonic shear source (Adapted from [30]). 
The final technique uses magnetic resonance elastography (MRE) to detect small tissue displacements. This method combines a mechanically applied stress (using a mechanical actuator or the acoustic radiation force of an ultrasound pulse) with MRI to measure displacement within the tissue or object. MRE usually combines MRI with mechanical waves to create a visual map, or elastogram, which can measure the stiffness of the body $[42,46]$. Moreover this is a non-invasive imaging modality that allows for the determination of the shear modulus (elastic modulus, shear stiffness) tissues in vivo.

Disadvantages of this technique are the long scan times, the acquisition of large amounts of data and the sophisticated reconstruction algorithms including phase unwrapping and stiffness inversions to produce measurements of material properties. The time required to acquire and analyze the data depend on the amount of data and the type of algorithm.

\subsection{OCT elastography to detect shear wave propagation and previous work}

Tissue elasticity may be thought of simply as the relative hardness or softness of a biomaterial, and clinical examination by palpation is a traditional and highly effective means of detecting pathologies such as coronary artery disease, which demonstrates local elastic inhomogeneity. OCT provides greater spatial and phase resolution than previous methods that have been used for the study of the deformation of tissue and biomaterials. The spatial resolution of mechanical property maps will depend on whether reliable phase difference measurements between locations can be made. It is expected that these maps have better spatial resolution compared to the shear wave wavelength. 
Optical coherence elastography (OCE) has been developed by many investigators within the last decade, including: J. M. Schmitt, A. Boppart, B. E. Bouma, D. D. Duncan, D. D. Sampson, S. G. Adie, X. Liang, B. F. Kennedy and their group.

A review of the development of OCE has been written by Wang and Larin [49]. During OCE, tissues can be excited internally or externally, as well as statically or dynamically [51].

Investigators have used OCE [47-50] to detect tissue motion. ARF was used for the internal mechanical excitation of a sphere embedded in a gelatin phantom, and the phantom deformations were detected with an OCT system and recorded as M-mode phase images. The displacement of the sphere over time was used for shear modulus measurements [51]. Tissue velocity and strain measurements have also been obtained via tissue imaged under mechanical loading with a vascular OCE protocol specific to the exploring of tissue biomechanics [52]. Strain responses of tissue phantoms undergoing compressive forces have been measured using speckle tracking and OCT methods for detecting small and large deformations [53]. Spectroscopic OCE (S-OCE) has been utilized for frequency-dependent contrast of the displacement amplitude and phase of a silicone phantom, with ex vivo tumor follow-up imaging, in B-mode OCT imaging with applications in pathology [54]. As well, a dynamic SDOCE technique applied to three-layer silicone tissue phantoms and ex vivo rat tumor tissue has been reported to provide contrast between sample regions with different mechanical properties, thus to mechanically characterize tissue [55]. In vivo three-dimensional OCE has also been implemented to observe elastic properties of superficial skin, which can be utilized 
for detecting strain rates and contrast useful for pathologists [56]. A ring actuator has also been applied to in vivo dynamic OCE to enable excitation and imaging for the same side of the sample, thus providing an alternative for contrast in OCT images. While these publications demonstrate a wide range of applications of OCE imaging in-vitro and clinically, none of them directly measure shear wave propagation.

In the first part of the thesis, we present a novel technique for calculating tissue mechanical properties by propagating shear waves in tissue equivalent phantoms and carotid artery samples with ARF, and measuring the shear wave speed and its associated properties with OCT phase maps. The OCT phase maps are acquired with a swept-source OCT (SSOCT) system. The higher phase noise exhibited by SS-OCT systems, compared to SD-OCT systems, generally limits the measurements of phase changes. This limitation is especially problematic at high A-scan rates, however noise of the relatively low speed SS-OCT (8kHz bi-directional) used in these experiments was sufficient to measure phase changes induced by shear wave propagation $[57,58]$. The dynamic excitation of the OCE technique uses ARF as the excitation source. Shear modulus measurements of tissue equivalent phantoms and carotid artery samples were made from OCT phase elastograms, without the requirement of measuring displacements of embedded targets as in [9]. We present this methodology as Shear Wave OCE (SW-OCE), a methodology I first introduced in my M.Sc. work. The use of ARF to induce the mechanical wave for these experiments was advantageous for several reasons including: a) the non-invasive nature of ARF excitation to induce deformations in depth in tissue and b) the control of the ultrasound transducer geometrical characteristics and frequen- 
cy which can induce a wide variety of shear waves (with different frequencies) that would enable probing the tissues at different spatial and temporal scales. Also, depending on the specific mechanical wave driver, consistent coupling of the driver to the tissue is a difficult issue to resolve which can result in a large variability in the measurements.

There are resolution and sensitivity advantages when using ARF excitation and SWOCE for shear wave propagation detection. SW-OCE utilizes OCT technology, which provides resolution advantages when compared to ultrasound or MRI. OCT provides higher spatial resolution (and typically temporal resolution), which allows for the detection of minute deformations in the phantoms that may be critical to the measurement of tissue mechanical properties. It is expected that these parametric maps of shear modulus have much better spatial resolution compared to the shear wave wavelength, in the same way that Fink and colleagues [59] achieve high resolution ultrasound images of shear wave properties using what they term "wave-to-wave" imaging, the difference here being that OCT is used instead of ultrasound imaging to track the shear waves.

In the beginning of the OCE study, we first showed the feasibility of optical coherence elastography measurements of shear wave propagation in homogeneous tissue equivalent phantoms and the method was validated by an independent measurement [7]. We were first group to detect shear wave propagation and developed a novel technique for calculating tissue mechanical properties by propagating shear waves in tissue/phantoms with ARF [7]. In this work for my Master's thesis, I explored the potential of measuring shear wave propagation using optical coherence elastography (OCE) based on a swept-source optical coher- 
ence tomography (OCT) system. Shear waves were generated using a $20 \mathrm{MHz}$ piezoelectric transducer (circular element $8.5 \mathrm{~mm}$ diameter) transmitting sine-wave bursts of $400 \mu \mathrm{s}$, synchronized with the OCT swept source wavelength sweep. The acoustic radiation force (ARF) was applied to two gelatin phantoms. Differential OCT phase maps, measured with and without the ARF, demonstrate microscopic displacement generated by shear wave propagation in these phantoms of different stiffness. I showed results of OCT derived shear wave propagation velocity and modulus, and compared these results to rheometer measurements, validating the technique. I showed that the mechanical excitation produces motions within the phantom that can be used for the estimation of mechanical properties using SW-OCE. This excitation produces shear waves that propagate perpendicular to the US beam. In this thesis, I have developed this technique to measure shear wave propagation using optical coherence elastography (OCE) in an inhomogeneous phantom and carotid artery samples based on a swept-source optical coherence tomography (OCT) system that is described in Chapter 2. I have demonstrated a SW-OCE technique that uses ARF for the mechanical excitation of a carotid sample to measure the shear modulus and Young's modulus of the adventitia. The mechanical excitation produces motions within the sample that can be used for the estimation of mechanical properties using SW-OCE. This study demonstrated the feasibility of shear wave OCE measurements in layered media. For the tissue equivalent phantoms, I have shown how measurements of mechanical properties can be made to differentiate between the boundaries of two media with different stiffness. It is expected that such differences in mechanical properties will occur at the transition region between a normal and diseased artery 
wall. In the carotid measurements presented in this thesis, the data collected suggest that the shear waves produced were confined to the adventitia of the carotid artery. This is due to the limited penetration depth of the OCT imaging and the $10 \mu \mathrm{m}$ axial resolution that can be achieved with this imaging modality. The measurement of the elastic properties of the adventitia demonstrates how the superior spatial resolution of OCT can be used in the SW-OCE approach to interrogate tissue structures that are too small to be resolved with other competing imaging methods. We present the feasibility of OCE measurements of shear wave propagation in tissue equivalent phantoms and carotid artery samples as a stepping stone for expanding the research into observing SW-OCE for investigating tissue mechanical properties in vivo.

\subsection{Drug delivery and enhanced transport of nanoparticles using ARF}

The second part of the thesis exploits the displacements induced by the acoustic radiation force to enhance the transport of nanoparticles in tissue equivalent materials (collagen matrix), with goal the enhanced displacement of nanoparticle transport in tumors.

It has been well established that blood vessels in tumors are abnormal [60]. Tumor vessel structure is a defective, and tumor vessels have a leaky endothelium. The transport of nanoparticles through a leaky tumor vessel wall and into the interstitial space surrounding cancer cells, resulting in the accumulation of nanoparticles in the tumor, is called the enhanced permeability and retention effect. For this to occur, the nanoparticles must exit through the pores of the leaky tumor vessels while at the same time not passing through the pores of the normal vessels [61]. The nanoparticles should be able to pass through the open- 
ings of the tumor vessels ranges can be up to $600 \mathrm{~nm}$ [62]. Therefore, any drug/particles being designed to leak through the tumor vasculature into the tumor parenchyma should be less than $600 \mathrm{~nm}$ to reach tumor tissues, and preferably smaller [63]. There are different types of nanoparticles which have been used as drug delivery vehicles such as polymeric micelles, liposomes, nanoemulsion droplets, metallic nanospheres, rods, shells and cages. Gold nanoparticles (GNPs) have attracted a great deal of attention as drug carriers since they can be imaged with multiple imaging modalities [64,65].

Particle size plays a significant role in the therapeutic efficacy of the gold nanoparticle approaches due to the preferential cellular uptake of gold nanoparticles of particular sizes. Gold nanoparticles typically range in size from 2 to $100 \mathrm{~nm}$. In-vitro studies have shown an optimal particle sizes of 40-50 $\mathrm{nm}$ to maximize uptake into cells [66-67]. However, once invivo, these nanoparticles have to travel through the tumor extracellular matrix before being taken up by a cell [68]. This transport of nanoparticles through the extracellular matrix determines the spatio-temporal distribution of the nanoparticles within the tissue [69]. The predominantly collagen mesh associated with the tumor extracellular matrix has the ability to significantly inhibit nanoparticle diffusion and transport to the tumor cells [70].

The extracellular matrix is a meshwork of proteins and carbohydrates that provides structural and biochemical support to the cells and connects tissues together. If the scaffold will later be placed in the body, such as in several applications in tissue engineering, it will need to be a biocompatible material [71]. 
There are different collagen types which are associated with various types of connective tissues in the body. Currently at least 18 types have been derived which vary in the length of the helix and the nature and size of the nonhelical portions [72-74]. Type I collagen is predominant in higher order animals especially in the skin, tendon, and bone where extreme forces are transmitted. Type II collagen is essentially unique to hyaline cartilage. Type III collagen is found in limited quantities in association with type I. Type IV is a highly specialized form found only in the basement membrane. Table 1.1 summarizes four different type of collagens found in animal tissues [75-77]. It should also be noted that the shear wave speed has been shown to be highly correlated to the collagen network in tissues [78,79]. Fig 1-8 shows Scanning Electron Micrographs (SEM) of electrospun collagens: type I, type II, type III and type IV.

Table 1.1. Different type of collagens found in animal tissues (Adapted from 75 and 76).

\begin{tabular}{|c|l|}
\hline Collagen Type & \multicolumn{1}{c|}{ Tissue distribution } \\
\hline I & $\begin{array}{l}\text { Skin, tendon, bone, cornea, dentin, fibrocartilage, large vessels, } \\
\text { intestine, uterus, dentin, dermis, tendon }\end{array}$ \\
\hline II & Hyaline cartilage, vitreous, nucleus pulposus, notochord \\
\hline III & $\begin{array}{l}\text { Large vessels, uterine wall, dermis, intestine, heart valve, gingiva (usually coexists with } \\
\text { type I except in bone, tendon, cornea) }\end{array}$ \\
\hline IV & Basement membranes \\
\hline
\end{tabular}



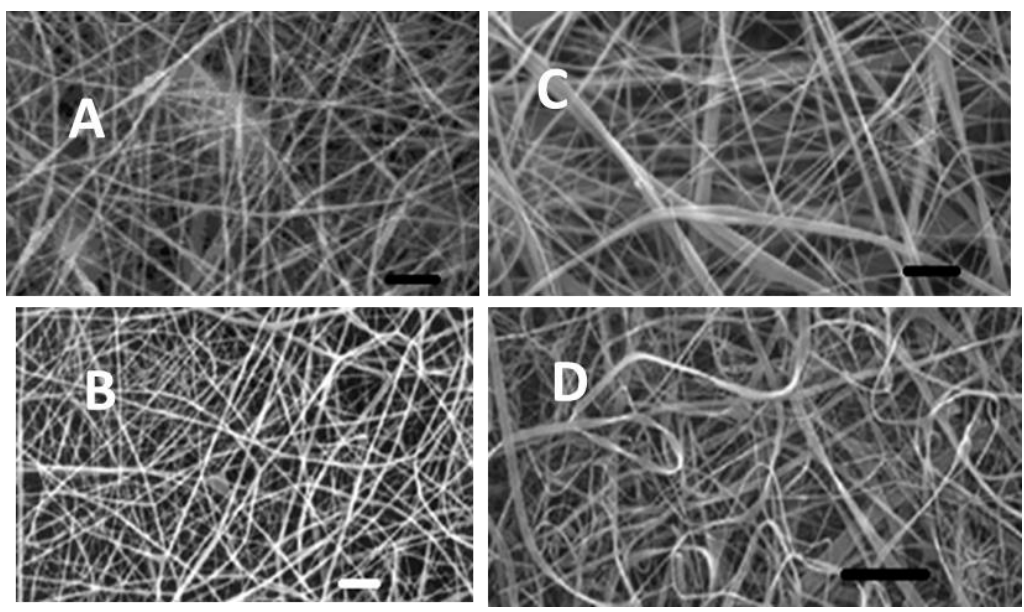

Figure 1-8 Scanning electron micrographs (SEM) of electrospun collagens type I (A), Collagen type II (B). Collagen type III (C) and collagen type IV (D). SEM scale bars $=1 \mu \mathrm{m}$ (A,B, and C) and $10 \mu \mathrm{m}$ (D). [ Adapted from 77, under the Creative Commons Attribution License (CC BY)].

Several investigators [80-83] have used GNP as contrast agents for optical coherence tomography (OCT). However, none of this work has investigated how OCT can be used to detect the enhanced penetration of $\mathrm{GNP}_{\mathrm{S}}$ through samples when they are exposed to an external force. In the second part of this thesis (Chapters 3 and 4), I demonstrated a technique to image the enhanced $\mathrm{GNP}_{\mathrm{s}}$ displacement generated using an acoustic radiation force (ARF).

\subsection{Hypothesis and specific aims}

The hypothesis in this thesis is that OCT can be used to image shear wave propagation in soft tissue with high resolution; be used to measure the mechanical properties of tissue; and the same methods used for the generation of the shear waves can be employed for the enhancement of the transport of gold nanoparticles (GNPs). The goal of this research is to develop shear wave based techniques that can image shear wave propaga- 
tion in inhomogeneous tissue equivalent phantoms and carotid samples; extract mechanical properties such as the shear modulus the and the Young's modulus; and also use OCT techniques to measure the enhanced displacements of nanoparticles in tissue equivalent samples as a stepping stone for expanding the research into observing enchantment of nanoparticle delivery in tumour tissues. To achieve this, we developed a method to measure the enhanced GNP displacement achieved using the ARF excitation after the injection of GNP into collagen phantoms with three different collagen concentrations: $3 \%, 6 \%$ and $10 \%$. The same ARF excitation was used to enhance the displacement of injected GNPs in a tissueengineered module, which contained MCF-7 breast cancer cells, that more closely emulates tumor tissue.

The specific aims of this proposed work are to:

a. Design experimental methods to create tissue deformation using an ARF excitation at scales that can be reliably detected using OCT.

b. Devise methods to measure the shear wave speed based on OCT M-mode and B-mode phase maps.

c. Devise methods to measure the enhanced transport of GNP using ARF based techniques by using SV-OCT methods.

\subsection{Overview of the Dissertation}

In Chapter 2, I proposed a method of measuring shear wave propagation using optical coherence elastography (OCE) in an inhomogeneous phantom and carotid artery samples based on a swept-source optical coherence tomography (OCT) system and then present the 
technique for calculating tissue mechanical properties by propagating shear waves in inhomogeneous tissue equivalent phantoms and carotid artery samples using the ARF of an ultrasound transducer. I measured the shear wave speed and associated mechanical properties in different layers with OCT phase maps. This work has been published in Biomedical optics express 5 (3), 895-906(2014).

In Chapter 3, I presented a technique to image the enhanced particle displacement generated using an acoustic radiation force (ARF) excitation source. Differential OCT speckle variance images with and without the ARF were used to estimate the particle displacement in a $10 \%$ collagen matrix. The images were used to detect the microscopic enhancement of nanoparticle displacement generated by the ARF. The experimental results are compared to simulation results and show that the displacement of the mesh induced by the ARF is of the same order of magnitude as the enhanced transport of the GNPs as measured with the OCT technique presented in this work. This work has been submitted to the journal Plos (Public Library of Science) one.

In Chapter 4, an acoustic radiation force (ARF) excitation source was used to enhance the transport of gold nanoparticles (GNPs) in a collagen matrix. The ARF was applied after nanoparticle injection and diffusion/transport into a collagen matrix, with and without the presence of cancer cells. Different concentrations of collagen (3\% and $6 \%)$ and a tissue engineered cellular construct, using MCF7 breast cancer cells embedded in a collagen matrix. Enhanced displacement of the GNPs was calculated in both the axial and lateral directions at 
locations close to the ultrasound focal point. This work has been submitted to the journal Biophotonics.

Chapter 5 summarizes the main results of the thesis and describes future work. 


\title{
Chapter 2: Optical coherence tomography detection of shear wave propagation in in- homogeneous tissue equivalent phantoms and ex-vivo carotid artery samples
}

\author{
Authors: Marjan Razani, Timothy W.H. Luk, Adrian Mariampillai, Peter Siegler,
} Tim-Rasmus Kiehl, Michael C. Kolios, and Victor X.D. Yang

M. Razani, T. W. H. Luk, A. Mariampillai, P. Siegler, T.-R. Kiehl, M. C. Kolios, and V. X. D. Yang, "Optical coherence tomography detection of shear wave propagation in inhomogeneous tissue equivalent phantoms and ex-vivo carotid artery samples" Biomed. Opt. Express

$$
\text { 5(3), 895-906 (2014). }
$$

\subsection{Abstract}

In this work, we explored the potential of measuring shear wave propagation using optical coherence elastography (OCE) in an inhomogeneous phantom and carotid artery samples based on a swept-source optical coherence tomography (OCT) system. Shear waves were generated using a piezoelectric transducer transmitting sine-wave bursts of $400 \mu$ s duration, applying acoustic radiation force (ARF) to inhomogeneous phantoms and carotid artery samples, synchronized with a swept-source OCT (SS-OCT) imaging system. The phantoms were composed of gelatin and titanium dioxide whereas the carotid artery samples were embedded in gel. Differential OCT phase maps, measured with and without the ARF, detected 
the microscopic displacement generated by shear wave propagation in these phantoms and samples of different stiffness. We present the technique for calculating tissue mechanical properties by propagating shear waves in inhomogeneous tissue equivalent phantoms and carotid artery samples using the ARF of an ultrasound transducer, and measuring the shear wave speed and its associated properties in the different layers with OCT phase maps. This method lays the foundation for future in-vitro and in-vivo studies of mechanical property measurements of biological tissues such as vascular tissues, where normal and pathological structures may exhibit significant contrast in the shear modulus.

Keywords: (170.4500) Optical coherence tomography; (170.6935) Tissue characterization.

\section{References and links}

1. J. Ophir, S. K. Alam, B. Garra, F. Kallel, E. Konofagou, T. Krouskop, and T. Varghese, "Elastography: ultrasonic estimation and imaging of the elastic properties of tissues," Proc. Inst. Mech. Eng. H 213(3),203- 233 (1999).

2. C. Sun, B. Standish, and V. X. D. Yang, "Optical coherence elastography: current status and future applications," J. Biomed. Opt. 16(4), 043001 (2011).

3. F. Sebag, J. Vaillant-Lombard, J. Berbis, V. Griset, J. F. Henry, P. Petit, and C. Oliver, "Shear wave elastography: a new ultrasound imaging mode for the differential diagnosis of benign and malignant thyroid nodules," J. Clin. Endocrinol. Metab. 95(12), 5281-5288 (2010).

4. R. Z. Slapa, A. Piwowonski, S. W. Jakubowski, K. T. Szopinski, J. Slowinska-Srzednicka, B. Migda, and R. K. Mlosek, "Shear wave elastography may add a new dimension to ultrasound evaluation of thyroid nodules: case series with comparative evaluation," J Thyroid Res. 657147(2012).

5. G. Scarcelli, S.H. Yun, "Confocal Brillouin microscopy for three-dimensional mechanical imaging," Nat. Photonics. 2(1), 39-43 (2008).

6. S. Shah, M. Laiquzzaman, R. Bhojwani, S. Mantry, I. Cunliffe, "Assessment of the biomechanical properties of the cornea with the ocular response analyzer in normal and keratoconic eyes," Invest. Ophthalmol. Vis. Sci. 48(7), 3026-3031(2007).

7. J. Li, S. Wang, K. R. Manapuram, M. Singh, F. M. Menodiado, S. Aglyamov,S. Emelianov, M. D. Twa, and K. V. Larina," Dynamic optical coherence tomography measurements of elastic wave propagation in tissue-mimicking phantoms and mouse cornea in vivo," J. Biomed. Opt. 18 (12), 121503 (2013).

8. J. M. Schmitt, "OCT elastography: imaging microscopic deformation and strain of tissue," Opt. Express 3(6), 199-211 (1998). 
9. X. Liang, M. Orescanin, K. S. Toohey, M. F. Insana, and S. A. Boppart, "Acoustomotive optical coherence elastography for measuring material mechanical properties," Opt. Letters 34(19), 2894-2896 (2009).

10. J. F. Greenleaf, M. Fatemi, and M. Insana, "Selected methods for imaging elastic properties of biological tissues," Annu. Rev. Biomed. Eng 5, 57-78 (2003).

11. J. G. Fujimoto, M. E. Brezinski, G. J. Tearney, S. A. Boppart, B. E. Bouma, M. R. Hee, J. F. Southern, and E. A. Swanson, "Optical biopsy and imaging using optical coherence tomography," Nature Medicine 1, 970-972 (1995).

12. S. Song, Z. Huang, and R. K. Wang, "Tracking mechanical wave propagation within tissue using phasesensitive optical coherence tomography: Motion artifact and its compensation," J. Biomed. Opt. 8(12), 121505 (2013).

13. S. Wang, S. Aglyamov, A. Karpiouk, J. Li, S. Emelianov, F. Manns, and K. V. Larin, "Assessing the mechanical properties of tissue-mimicking phantoms at different depths as an approach to measure biomechanical gradient of crystalline lens," Biomed. Opt. Express. 4(12), 2769-2780 (2013).

14. A. P. Sarvazyan, O. V. Rudenko, S. D. Swanson, J. B. Fowlkes, and S. Y. Emelianov, "Shear wave elasticity imaging: A new ultrasonic technology of medical diagnostics," UMB 24(9), 1419-1435 (1998).

15. M. F. O'Rourke, J. A. Staessen, C. Vlachopoulos, D. Duprez, G. E. Plante, "Clinical applications of arterial stiffness; definitions and reference values," Am J Hypertension 15, 426-444 (2002).

16. K. Cheng, C. R. Baker, G. Hamilton, A. P. G. Hoeks, A. M. Seifalian, "Arterial elastic properties and cardiovascular risk/event," Eur J Vasc Endovasc Surg 24, 383-397 (2002).

17. S. Laurent, P. Boutouyrie, R. Asmar, I. Gautier, B. Laloux, L. Guize, P. Ducimetiere, A. Benetos, "Aortic stiffness is an independent predictor of all-cause and cardiovascular mortality in hypertensive patients," Hypertension 37, 1236-1241 (2001).

18. M. Couade, M. Pernot, C. Prada, E. Messas, J. Emmerich, P. Bruneval, A. Criton, M. Fink, M. Tanter, "Quantitative assessment of arterial wall biomechanical properties using shear wave imaging," Ultrasound Med. \& Biol. 36, 1662-1676 (2010).

19. G. Pasterkamp and E. Falk, "Atherosclerotic plaque rupture: an overview,” J. Clin. Basic Cardiol. 8186 (2000).

20. E. Falk, "Why do plaques rupture," Circulation 86, 30-42 (1992).

21. K. C. Hilty and D.H Steinberg, "Vulnerable plaque imaging-current techniques," J. Cardiovasc. Trans. Res. 2, 9-18 (2009).

22. F. Sharif and R. T. Murphy, "Current status of vulnerable plaque detection," Cath. Cardiovasc. Interven. 75, 135-144 (2010).

23. C. Schmitt, G. Soulez, R. L. Maurice, M. F. Giroux, and G. Cloutier, "Noninvasive vascular elastography: toward a complementary characterization tool of atherosclerosis in carotid arteries," Ultrasound Med. Biol. 33(12), 1841-1858 (2007).

24. J. J. Dahl, D. M. Dumont, J. D. Allen, E. M. Miller, and G. E. Trahey, "Acoustic radiation force impulse imaging for noninvasive characterization of carotid artery atherosclerotic plaques: a feasibility study," Ultrasound Med Biol. 35(5), 707-716 (2009).

25. M. Fatemi and J. F. Greenleaf, "Application of radiation force in noncontact measurement of the elastic parameters," Ultrason. Imaging 21(2), 147-154 (1999).

26. M. Elkateb Hachemi, S. Callé, and J. P. Remenieras, "Transient displacement induced in shear wave elastography: comparison between analytical results and ultrasound measurements," Ultrasonics 44(Suppl 1), e221-e225 (2006).

27. L. Ostrovsky, A. Sutin, Y. Il'inskii, O. Rudenko, and A. Sarvazyan, "Radiation force and shear motions in inhomogeneous media," J. Acoust. Soc. Am 121(3), 1324-1331 (2007). 
28. M. L. Palmeri, S. A. McAleavey, K. L. Fong, G. E. Trahey, and K. R. Nightingale, "Dynamic mechanical response of elastic spherical inclusions to impulsive acoustic radiation force excitation," IEEE Trans. Ultrason. Ferroelectr. Freq. Control 53(11), 2065-2079 (2006).

29. W. F. Walker, F. J. Fernandez, and L. A Negron, "A method of imaging viscoelastic parameters with acoustic radiation force," Phys.Med. Biol 45(6), 1437-1447 (2000).

30. K. Nightingale, M. S. Soo, R. Nightingale, and G. Trahey, "Acoustic radiation force impulse imaging: In vivo demonstration of clinical feasibility," Ultrasound Med Biol 28(2), 227-235 (2002).

31. S. H. Yun, G. J. Tearney, J. F. de Boer, and B. E. Bouma, "Motion artifacts in optical coherence tomography with frequency-domain ranging," Opt. Express 12(13), 2977-2998 (2004).

32. K. R. Nightingale, R. W. Nightingale, D. L. Stutz, and G. E. Trahey, "Acoustic radiation force impulse imaging of in vivo vastus medialis muscle under varying isometric load," Ultrason. Imaging 24(2), 100-108 (2002).

33. M. Fatemi and J. F. Greenleaf, "Ultrasound-stimulated vibro-acoustic spectrography," Science 280(5360), 82-85 (1998).

34. J. D. Allen, K. L. Ham, D. M. Dumont, B. Sileshi, G. E. Trahey, and J. J. Dahl. "The development and potential of acoustic radiation force impulse (ARFI) imaging for carotid artery plaque characterization," Vasc. Med. 16, 302-1 (2011).

35. M. Razani, A. Mariampillai, C. Sun, T. W. H. Luk, V. X. D. Yang, and M. C. Kolios, "Feasibility of optical coherence elastography measurements of shear wave propagation in homogeneous tissue equivalent phantoms," Biomed. Opt. Express 3(5), 972-980 (2012).

36. D. C. Adler, R. Huber, and J. G. Fujimoto, "Phase-sensitive optical coherence tomography at up to 370,000 lines per second using buffered Fourier domain mode-locked lasers," Opt. Letters 32(6), 626628 (2007).

37. M. W. Urban and J. F. Greenleaf, "Kramers-Kronig based quality factor for shear wave propagation in soft tissue," Phys Med Biol 54(19), 5919-5933 (2009).

38. C. Amador, M. W. Urban, S. Chen, Q. Chen, K-N. An, and J. F. Greenleaf, "Shear elastic modulus estimation from indentation and SDUV on gelatin phantoms," IEEE Trans. Biomed. Eng. 58(6), 1706 $1714(2011)$.

39. V. X. D. Yang, M. L. Gordon, B. Qi, J. Pekar, S. Lo, E. Seng-Yue, A. Mok, B. C. Wilson, and I. A. Vitkin, "High speed, wide velocity dynamic range Doppler optical coherence tomography (Part I): System design, signal processing, and performance," Opt. Express 11(7), 794-809 (2003).

40. S. L. Floc'h, G. Cloutier, G. Finet, P. Tracqui, R. I. Pettigrew, and J. Ohayon, "On the potential of a new IVUS elasticity modulus imaging approach for detecting vulnerable atherosclerotic coronary plaques: in vitro vessel phantom study," Phys. Med. Biol. 55, 5701-5721 (2010).

41. H. Kanai, H. Hasegawa, M. Ichiki, F. Tezuka, and Y. Koiwa, "Elasticity imaging of atheroma with transcutaneous ultrasound - preliminary study," Circulation 107, 3018-3021 (2003). 


\subsection{Introduction}

Elastography is a method for analyzing the biomechanical properties of tissues based on stiffness and strain images [1]. The biomaterial tissue deformations caused by static or dynamic loads are recorded in elastograms that contain data about the local variations of the stiffness inside a region of interest [2]. This information is valuable for identifying and assessing biomaterial variations of the target tissue. The shear modulus of biomaterials in particular is thought to be highly sensitive to variations in the biomaterial properties. There are many different imaging modalities that can be used to measure displacements and estimate the resulting mechanical properties. Two of these are ultrasound (US) and magnetic resonance imaging (MRI). However, they come with significant drawbacks, such as high cost and complex design. They also have insufficient spatial resolution to detect small scale and subtle elastic variations in biomaterials and tissues, such as in tissue engineered modules and atherosclerotic plaques. Supersonic shear weave elastography, which is a method that uses a shear source moving through the medium at a supersonic speed (greater than the speed of the shear wave in tissue) and is use to generate parametric images of shear modulus. This can be achieved by successively focusing the ultrasonic "pushing" ARF beam at different depths at a speed that is greater than the tissue shear wave speed. The result of the beam traveling faster than the shear wave is that all the resulting shear waves interfere constructively along a Mach cone creating planes of intense shear waves propagating in opposite directions, analogous to a "sonic boom" created by a supersonic aircraft $[3,4]$. A method based on Brillouin light-scattering and involving a spectral shift proportional to the longitudinal modulus 
of elasticity of the tissue allows for non-contact and direct readout of the viscoelastic properties of a material [5]. Other methods previously investigated to compare hysteresis in normal and keratoconic eyes using the Ocular Response Analyzer (ORA; Reichert Ophthalmic Instruments, Buffalo, NY). This device is an adaptation to the noncontact tonometer (NCT) to measure the viscoelastic properties of the eye [6]. Lastly, another method uses a focused airpulse system and a phase-stabilized swept-source optical coherence tomography to assess the propagation of low-amplitude (micron-level) waves [7].

In contrast, optical coherence tomography (OCT) has several advantages over other imaging modalities. Analogous to US, OCT uses light instead of sound waves, is inexpensive compared to other imaging modalities and has higher resolution and motion sensitivity to allow for improved identification of micron-sized morphological tissue structures and highly localized strains [8-10]. The main disadvantage of the technique is its limited penetration depth, on the order of few millimeters [11].

Optical coherence elastography (OCE) is a technique that uses OCT for measuring the biomechanical properties of soft tissues $[12,13]$. The tissues can be excited internally or externally, and statically or dynamically. A common method for creating dynamic compressions for the tissue is using acoustic radiation force (ARF) or low-frequency vibrations with a needle. Shear waves generated from the application of ARF will propagate away from the initial region of excitation (i.e. the focal spot region) [14]. Typical values for the shear wave propagation speed in soft tissues range from $1-10 \mathrm{~m} / \mathrm{s}$ and the attenuation coefficient is two to three orders of magnitude greater than that of compression waves [14]. Both of these pa- 
rameters are directly related to the mechanical properties of the tissue. Due to its high attenuation, the shear wave generated propagates within a limited area of tissue, typically a few millimeters from the location of the excitation [14]. The frequency of the shear wave will be determined primarily by the width of the ultrasound beam and not the time duration of the excitation, unless the excitation duration approaches the natural time constants of soft tissue relaxation.

The study of the mechanical properties of arteries is critical to understanding cardiovascular function and in the diagnosis of vascular disease [15]. Arterial stiffness increases with aging and can be used as an indicator for cardiovascular morbidity and mortality, as well as a measure for the risk of stroke and myocardial infarction [16-18]. These acute cardiovascular events typically occur when there is a rupture of the fibrous cap of the atherosclerotic plaque, causing the formation of a thrombus that obstructs an artery $[19,20]$. An atherosclerotic plaque includes a large extracellular necrotic core and a thin fibrous cap infiltrated by macrophages $[21,22]$. These components have properties that are important in quantifying lesion stability [23]. Specific to the carotid artery, atherosclerotic disease and plaque rupture are risk factors for stroke. ARF imaging can be applied to carotid artery samples to determine the feasibility of noninvasively characterizing atherosclerotic plaques [24].

In this work, we explore the potential for measuring shear wave propagation in inhomogeneous phantoms and excised carotid artery samples using OCT as an imaging modality to detect the shear wave propagation. Using OCE allows for the measurement of tissue displacement to generate high-resolution elastograms. 


\subsection{Methods}

$\mathrm{ARF}$ was used as the internal mechanical excitation to send focused ultrasound beams to produce the desired shear waves. The ARF was applied using a $20 \mathrm{MHz}$, circular, piezoelectric transducer element (PZT, f-number 2.35) transmitting sine-wave bursts of $400 \mu \mathrm{s}$ duration. The internal displacements induced by the shear waves were detected using a Thorlabs swept source OCT system and phase sensitive motion detection algorithms. The OCT laser had a center wavelength of a $1310 \mathrm{~nm}$, a bandwidth of $~ 110 \mathrm{~nm}$, and an A-scan rate of $8 \mathrm{kHz}$. The lateral resolution was approximately $13 \mu \mathrm{m}$ in the samples. Using this technique, mechanical properties of the phantoms were measured. The ARF excitation for producing transient excitations had previously been implemented to assess the mechanical properties of tissues [25-29]. ARF imaging had also been used in general elasticity imaging methods for the characterization of lesions, muscle screening, and imaging of the calcification of arteries [30-34].

Two types of samples were investigated in this study: an inhomogeneous tissue equivalent phantom and an ex-vivo bovine carotid artery embedded in gel. Four tissue equivalent phantoms with two layers were made. Each phantom was made using gelatin mixed with titanium dioxide as a scattering agent. Gelatin concentrations of $12 \%$ and $8 \%$ (indicated as high concentration and low concentration, respectively) were varied to make layers with differing stiffness values. Gelatin powder (Type B, Fisher Scientific, G7-500) and distilled water were heated in a water bath at $60-65{ }^{\circ} \mathrm{C}$ for one hour and stirred periodically. When the phantom samples cooled to $45{ }^{\circ} \mathrm{C}, 0.1 \%$ by weight titanium dioxide (Sigma- 
Aldrich, Titanium(IV) oxide nanopowder, $<25 \mathrm{~nm}$ particle size, $99.7 \%$ trace metals basis) was added and mixed thoroughly. The phantom solution was poured into rectangle molds (20 mm height) and allowed to congeal. The first layer was left to cool before the second layer was added on top of the first layer (as shown in Fig. 2.1). The inhomogeneous tissue equivalent phantom was rotated on the side and imaged.

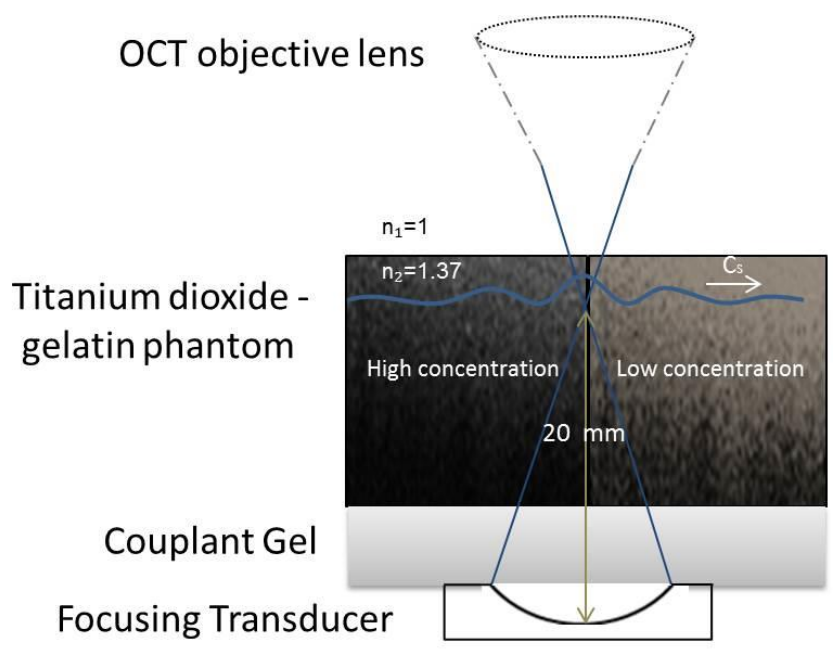

Figure 2-1A focused transducer was used to produce an ARF impulse to generate shear waves at the focal point of the transducer. The shear wave propagated in the inhomogeneous tissue equivalent phantom consisted of two layers that were labeled as hard (12\% concentration ) and soft ( $8 \%$ concentration).

A normal bovine carotid artery was obtained from a local butcher shop. The bovine carotid artery was cut into three sections and three phantoms were prepared in a similar fashion to the first phantom, but differed in the layering process. Instead of using gelatin of differing concentration as its second layer, a carotid artery sample was embedded above the first gelatin layer when the layer was cooled to $40{ }^{0} \mathrm{C}$ (as shown in Fig. 2.2). The experimental 
setup for the imaging of these two types of samples was described elsewhere and shown in Fig. 2.3 [35].

B-mode and m-mode OCT images were obtained using a commercial swept-source system (Thorlabs, Inc., USA). B-mode images of a sample (5 $\mathrm{mm}$ in length) were obtained at the A-scan depth of $3 \mathrm{~mm}$. The focal point of the transducer for the ARF was $20 \mathrm{~mm}$ in depth from the transducer surface. This focal point was located $1 \mathrm{~mm}$ below the top surface of the sample. M-mode images of this same sample were taken along the direction of the ARF beam. In this setup, shear waves predominantly propagate radially away from the focal point in the direction perpendicular to the direction of the ARF beam. 


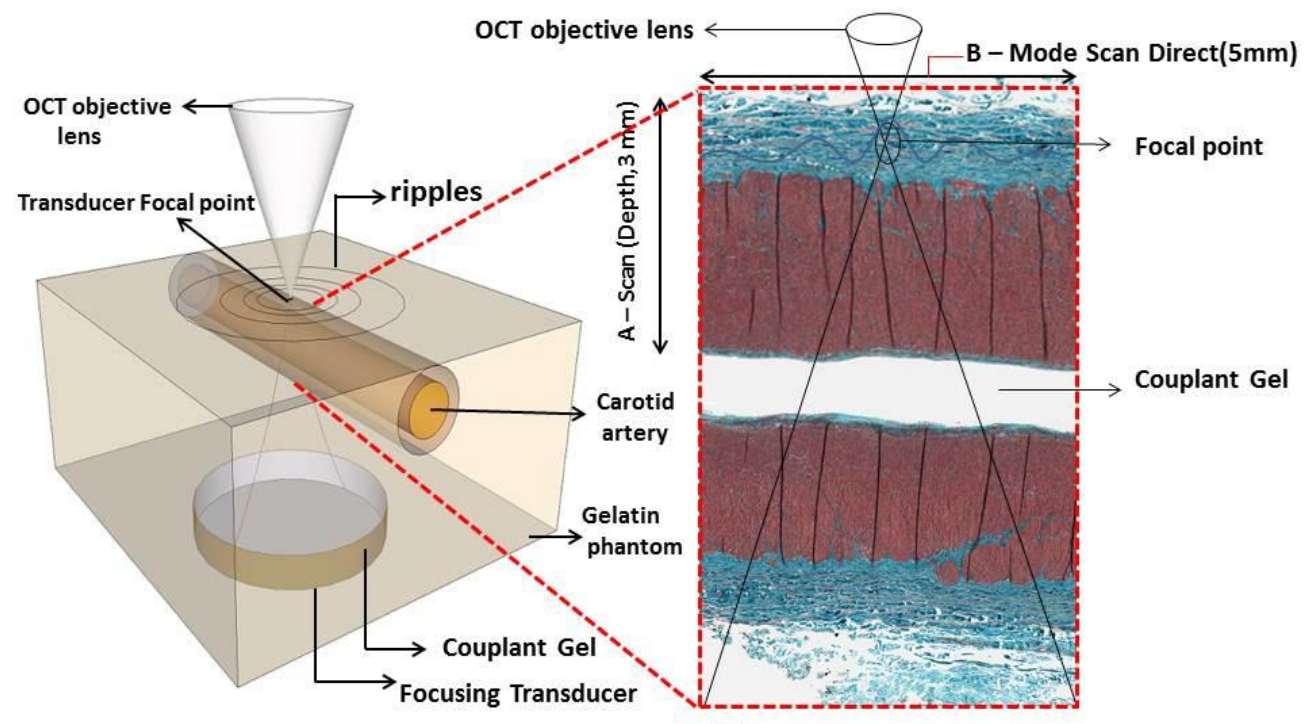

(a)

(b)

Figure 2-2 a) A focused transducer was used to produce an ARF impulse to generate shear waves at the focal point of the transducer, similar to Fig. 2.1. (b) The shear wave propagated in this sample consisted of the carotid artery embedded on top of the gelatin.

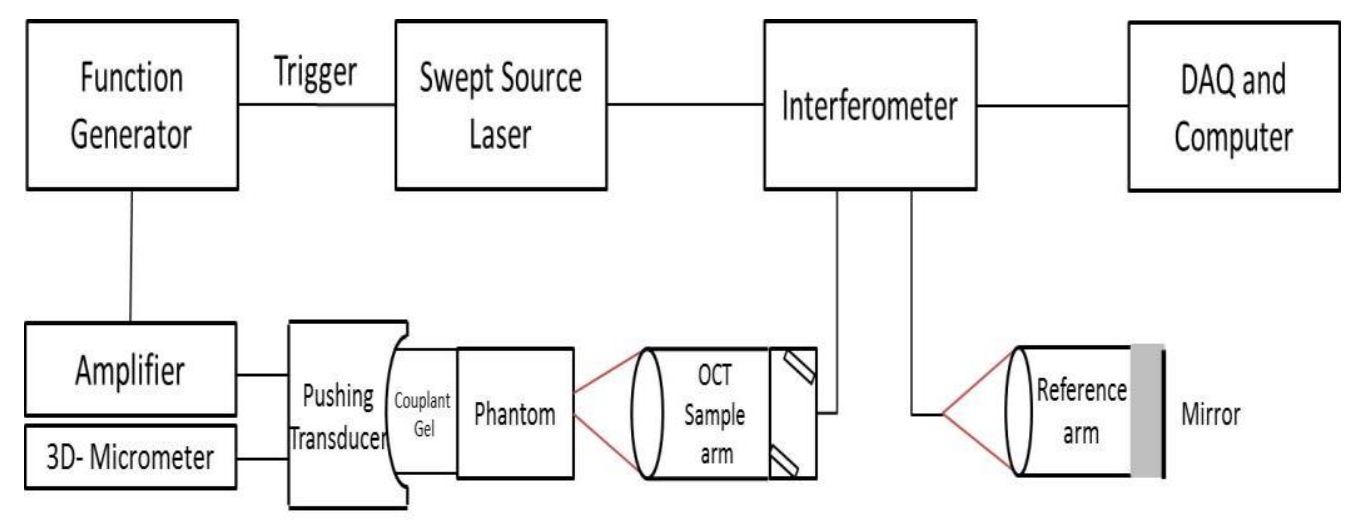

Figure 2-3 A schematic diagram of the ARF-OCE experimental setup. The setup consisted of the existing SSOCT system, an inhomogeneous titanium dioxide-gelatin phantom, a carotid artery embedded phantom, a focused pushing transducer (20 MHz, f-number 2.35), an amplifier, a 3D micrometer stage and a function generator (Agilent 33250A $80 \mathrm{MHz}$, Function / Arbitrary Waveform Generator) synchronized with the SS-OCT system. 
The attenuation of shear wave was calculated by the examination of the change in signal strength in the direction of propagation of the shear wave. The attenuation of the shear wave as it propagates through the phantom is given by Eq. (2.1):

$$
I=I_{0} \exp (-\alpha x)
$$

where $\mathrm{I}$ is the intensity of the shear wave that has propagated a distance $\mathrm{x}$ through the material, $I_{0}$ is the intensity of the shear wave at the focal spot and $\alpha$ is the shear wave attenuation coefficient. To calculate the attenuation coefficient, the signal strength at different locations in the phantom was obtained as a function of distance from the focal spot using the Peak displacement as an indicator of intensity $(x)$. The amplitude of the shear wave was obtained by averaging 512 A-lines to obtain the average value for $\alpha$. A best fit to the average amplitude as a function of lateral distance was obtained using the MATLAB function envelope for a lateral distance of $5 \mathrm{~mm}$. From this best fit, the shear wave attenuation coefficient was calculated. Shear wave speed can be calculated using Eq. (2.2):

$$
C_{S}(\omega)=\frac{\omega \Delta r}{\Delta \varphi}
$$

where $\omega=2 \pi f, \Delta \varphi$ is the phase shift and $\Delta r$ is the distance between the two successive lateral distance points. OCT images of the inhomogeneous phantoms were taken with the SS-OCT system and B-mode phase maps were obtained. These images provided information to track the phase shift $(\Delta \varphi)$ at two successive lateral distance points $(\Delta \mathrm{r})$ for a given depth $\mathrm{z}$ from which the shear wave speed was calculated. This was done for each pair of points in the image choosing the distance $\Delta \mathrm{r}$ as $0.5 \mathrm{~mm}$ and all pairs of points in the image. 
After obtaining the speed maps, they are then converted to shear modulus maps using Eq. (2.3):

$$
C_{S}=\sqrt{\frac{\mu}{\rho}} .
$$

where $\mu$ represents the shear modulus and $\rho$ is density of the sample, resulting in Fig.

2.4(c)

\subsection{Results}

OCT images of the inhomogeneous phantoms were obtained with the SS-OCT system and are shown in Fig. 2.4. The B-mode images, as well as their respective phase and displacement maps, allowed the calculation of distance between locations in the image, the phase shift between these locations, and the sample displacement caused by the shear wave.
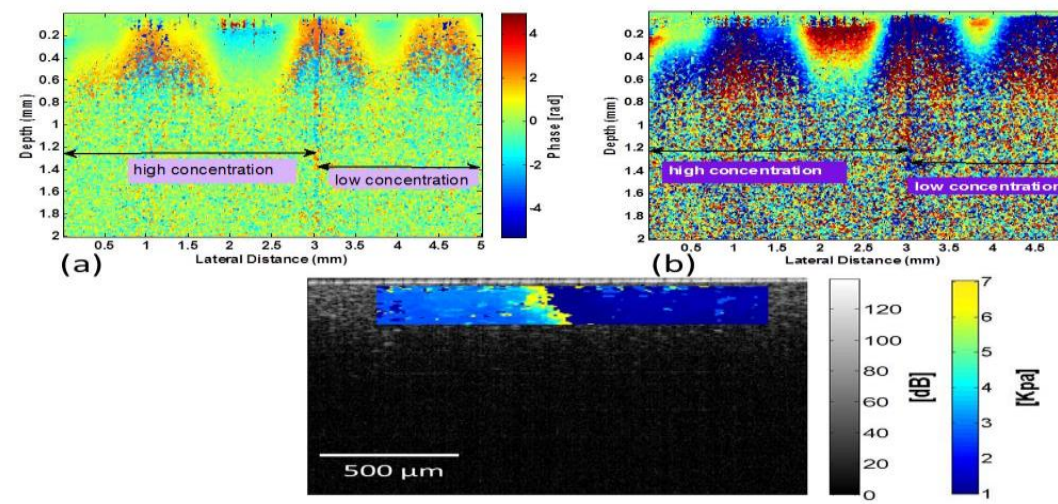

(c)

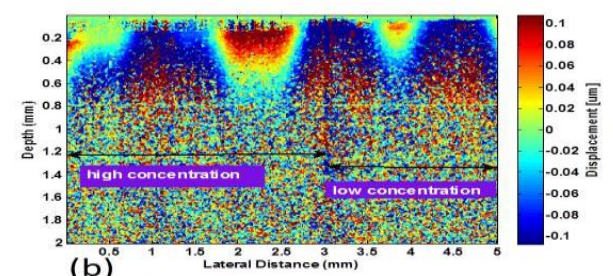


OCT images of the inhomogeneous phantoms were taken with the SS-OCT system and B-mode phase maps were obtained. These images provided information to calculate the distance between two measurement points $(\Delta r)$ and the phase shift $(\Delta \varphi)$ between these two tracking locations for each layer which two successive locations can be chosen at a particular depth $\mathrm{z}$ (in this work we chose $\mathrm{z}=0.2 \mathrm{~mm}$ in the regions of high concentration and also in the regions of low concentration). At these two locations in the image, phase values are retrieved, then were in turn used to calculate the shear wave speed, shear modulus and Young's modulus [28]. The optical path displacement $Z$ is calculated from the measured phase by using Eq. (2.4) [36]:

$$
Z=\frac{\lambda_{0} \Delta \varphi}{4 \pi n} .
$$

where $\lambda_{0}$ is the center wavelength and $n$ is the sample refractive index. In Fig.2. 4(b) the $\mathrm{x}$-axis is the lateral distance within the phantom and the $\mathrm{y}$-axis is the depth. The color in the image represents the displacement calculated from the phase maps in Fig. 2.4(a).

In Fig. 2.4(c), a shear modulus map (color scale) is superimposed on the B-mode image. The parametric images show the difference between the two sides of the phantom, with artifacts at the boundary due to the boundary discontinuity.

The image intensity along a profile through the ultrasound focus was plotted for 4 different phase offsets between the mechanical excitation and the OCT from 0 to $2 \pi$. The image intensity profiles of all phase offsets were composed in one diagram to estimate the attenuation of the mechanical wave amplitude. Fig. 2.5 shows a succession of images at different phase offsets illustrating the shear wave propagation in the tissue equivalent phantom. 


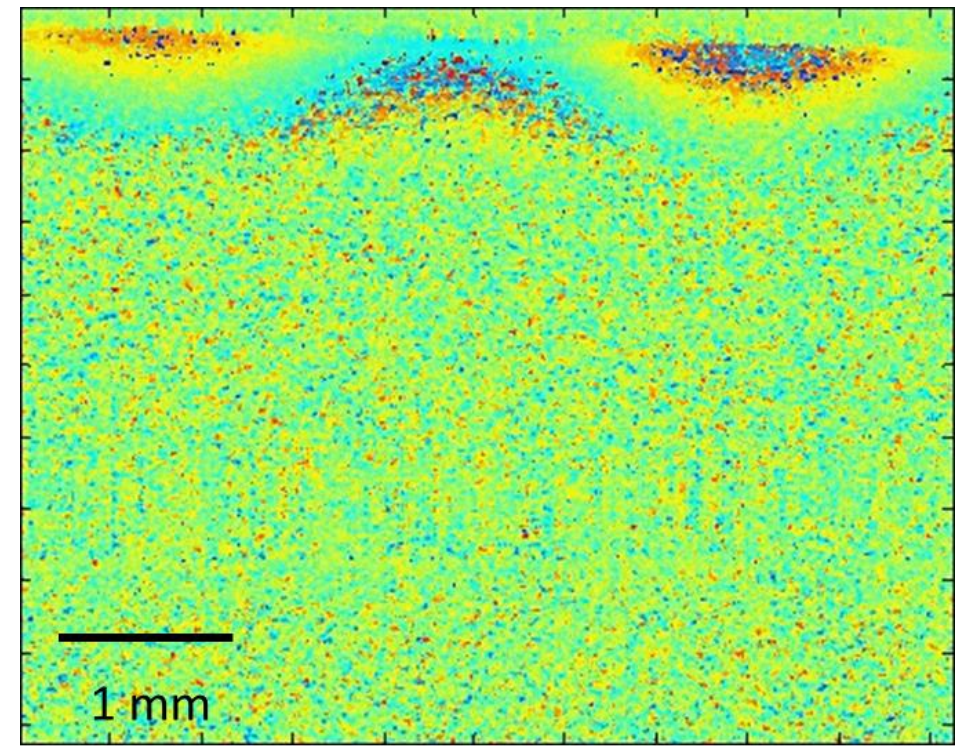

Figure 2-5 A movie of the shear wave propagation (Media 1) for the tissue equivalent phantom which was created by using different phase offsets (time delays) between the Focusing Ultrasound (FUS) and OCT. The X axis and $\mathrm{Y}$ axis represent lateral distance $(5 \mathrm{~mm})$ and depth $(3 \mathrm{~mm})$ respectively. The color scale represent the phase, as in Fig. 2.4(a) (rad).

The shear wave attenuation coefficient was estimated by measuring the damping of the shear wave as it propagated away from the ARF focal spot. The focal spot was located at the boundary between the two layers of different concentrations (at around $\mathrm{x}=3 \mathrm{~mm}$ in Fig. 2.4(a))

The attenuation coefficient was approximately $0.8 \mathrm{~Np} / \mathrm{cm}$, which is within the range of previously published data for gelatin phantoms [37].This was done for all four phantoms.

OCT images of three normal bovine carotid arteries samples were taken with the SSOCT system and shown in Fig. 2.6. 

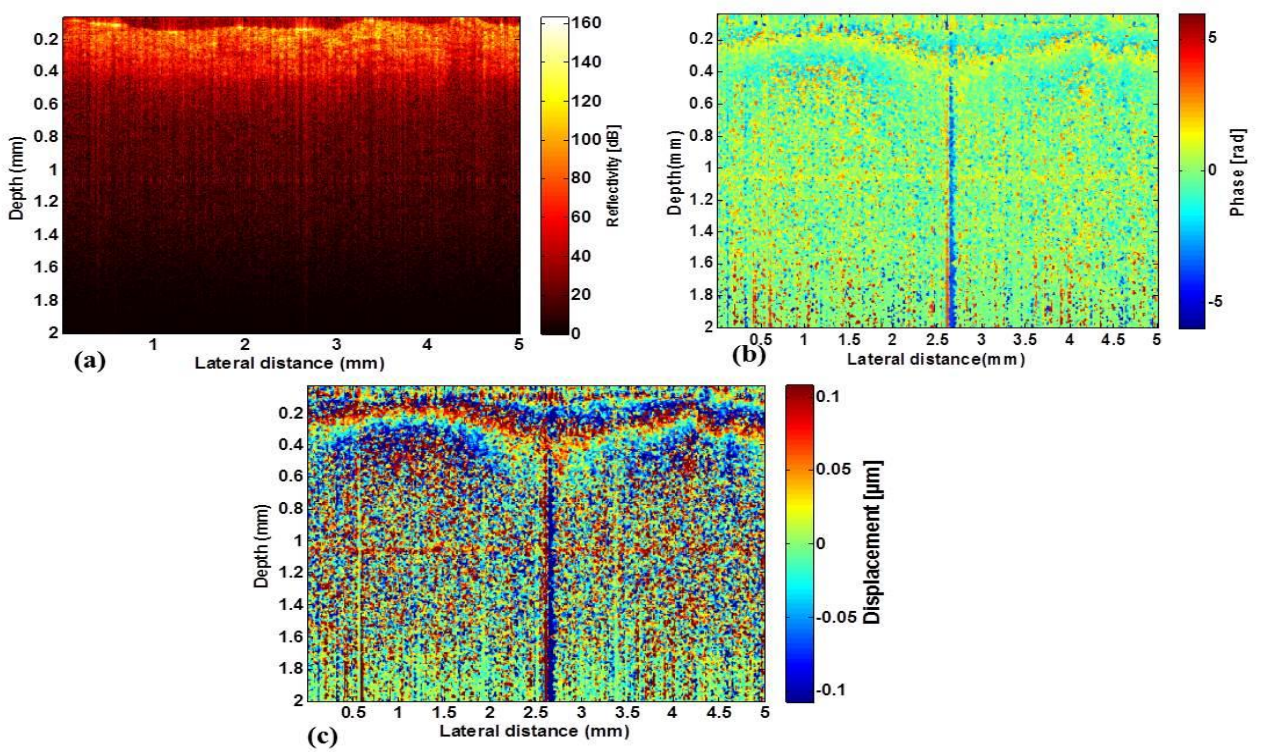

Figure 2-6B-mode OCT structural images (a) and the corresponding B-mode phase map (b) of the carotid artery samples were obtained with the SS-OCT system. The color scale represents the change of the phase value (radians). (c) The displacement of the sample from its initial position was measured.

After imaging with OCT, the carotid artery samples were fixed in formalin for 48 hours. Hematoxylin and Eosin (H\&E) as well as Masson trichrome stains were performed, followed by whole-slide scanning (ImageScope, Aperio, Vista, CA) for quantitative digital pathology assessment of the carotid artery samples.

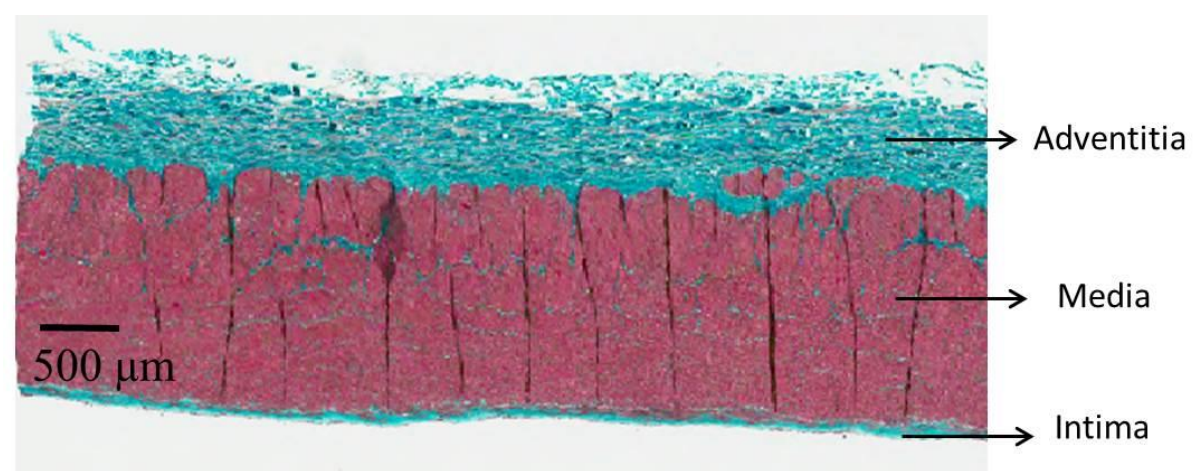

Figure 2-7Masson trichrome stain histology image of one of the ex-vivo carotid artery samples. 
The Masson trichrome stain histology images of the ex-vivo carotid artery sample, shown in Fig.2. 7, were correlated with the phase maps of the sample. The results from these images suggest that shear waves were generated in the adventitia layer. Therefore, the mechanical properties measured are likely representative of this tissue layer.

The dominant frequency of the shear wave was calculated to be approximately 266 $\mathrm{Hz}$ (for both the samples). The shear wave group speed was then calculated by using the $\Delta r$ and $\Delta \varphi$ obtained from the phase map, which depicts the distance between the two measurement locations and the calculated phase shift, respectively. These values were used to calculate the shear modulus and young modulus [35]. The mechanical properties of the inhomogeneous phantoms and carotid artery samples are shown in Table 2.1. The average values and standard deviations were calculated from 10 different pairs of locations in the phase maps for all calculations of $\Delta r$ and $\Delta \varphi$. The errors for the SW-OCE results represent the standard deviation of these 10 measurements, for all 4 phantoms. The three phantoms with the bovine carotid arteries were imaged, and the results report the average and standard deviation of the 3 measurements. As expected, the values of the Young's moduli and shear moduli were greater for the part of the phantom with the higher concentration of gelatin $[35,38]$. The shear modulus and young modulus of carotid artery sample were $45.3 \pm 0.6 \mathrm{kPa}$ and $135.8 \pm 1.8$ $\mathrm{kPa}$ respectively. 
Table 2.1 : The mechanical properties of the inhomogeneous phantoms and carotid artery samples (adventitia). The errors for the SW-OCE results represent the standard deviation as explained in the text.

\begin{tabular}{lccc}
\hline Samples & Shear wave speed & Shear modulus & Young's modulus \\
& $\left(C_{s}, m / s\right)$ & $(\mu, k P a)$ & $(E, k P a)$ \\
\hline inhomogeneous & $1.87 \pm 0.06$ & $3.7 \pm 0.2$ & \\
phantom (high & & & \\
concentration, & & $71.0 \pm 0.6$ \\
12\%) & & \\
\hline inhomogeneous & & \\
phantom & & \\
(low concentra- & & \\
tion, 8\%) & & \\
\hline Carotid artery & & \\
sample (adventi- & & \\
tia) & & \\
\hline
\end{tabular}

While many factors contribute, the phase stability of our OCT system during these imaging sessions are primarily affected by the signal-to-noise ratio (SNR). Therefore, we measured the phase stability as function of the SNR (Fig. 2,8), analogous to our previous work [3]. 


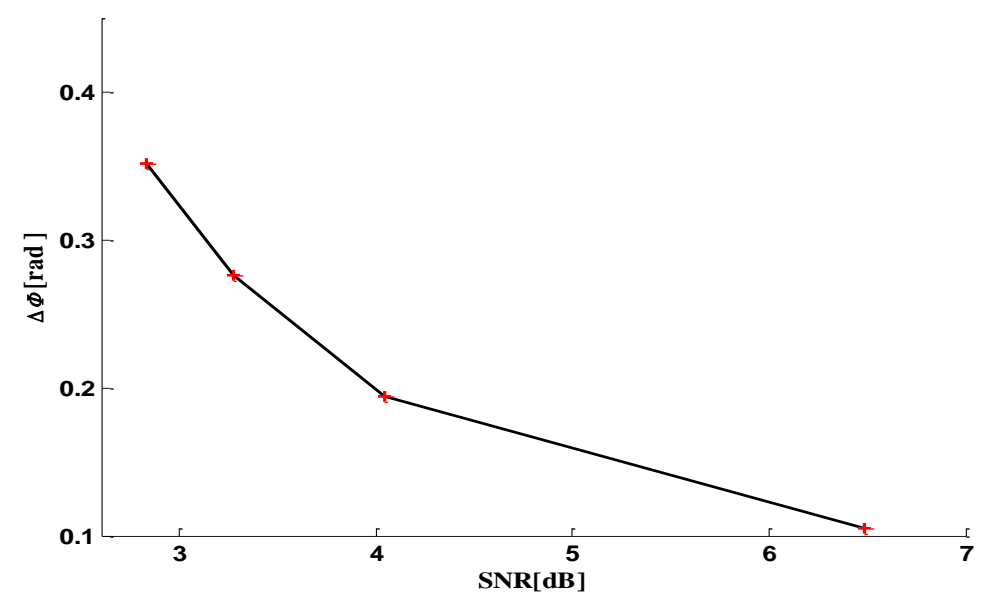

Figure 2-8 Mean background phase noise $(\Delta \varphi)$ at four different SNR regions.

\subsection{Discussion}

Shear waves were generated in inhomogeneous phantoms and in carotid artery samples. The shear modulus and Young's modulus were measured. We have demonstrated, for the first time, a SW-OCE technique that uses ARF for the mechanical excitation of a carotid sample to measure the shear modulus and Young's modulus of the adventitia. The mechanical excitation produces motions within the sample that can be used for the estimation of mechanical properties using SW-OCE. This study demonstrated the feasibility of shear wave OCE measurements in layered media. For the tissue equivalent phantoms, we have shown how measurements of mechanical properties can be made to differentiate between the boundaries of two media with different stiffness (Fig. 2.4). A parametric map of the shear modulus clearly shows the difference in the shear modulus in the low and high gel concentration regions (Fig. 2.4(c)). It is expected that such differences in mechanical properties will occur at the transition region between a normal and diseased artery wall. 
Moreover, in the carotid measurements presented in this paper, the data collected suggest that the shear waves produced were confined to the adventitia of the carotid artery. This is due to the limited penetration depth of the OCT imaging and the $10 \mu \mathrm{m}$ axial resolution that can be achieved with this imaging modality. The values measured in Table 2.1 compare well to published values using different techniques such as B-mode echography [40], intravascular ultrasound [41]. Young's modulus measurements for the carotid artery lie between $30.1 \pm 10.8 \mathrm{kPa}$ and $614 \pm 35 \mathrm{kPa}$, and depend on many parameters such as species, age, diameter and disease [40]. The measurement of the elastic properties of the adventitia demonstrates how the superior spatial resolution of OCT can be used in the SW-OCE approach to interrogate tissue structures that are too small to be resolved with other competing imaging methods.

Tissue elasticity may be thought of simply as the relative hardness or softness of a biomaterial, and clinical examination by palpation is a traditional and highly effective means of detecting pathologies such as coronary artery disease, which demonstrates local elastic inhomogeneity. OCT provides greater spatial and phase resolution than previous methods that have been used for the study of the deformation of tissue and biomaterials. The spatial resolution of mechanical property maps will depend on whether reliable phase difference measurements between locations can be made with SW-OCE. It is expected that these maps have much better spatial resolution compared to the shear wave wavelength. 


\title{
Chapter 3: Feasibility of Acoustic Radiation Force-Optical Coherence Tomography for Visualization and characterization of en- hancement of gold nanoparticles
}

\author{
Authors: Marjan Razani, Azhar Zam, Nico J J Arezza, Michael C Kolios \\ Marjan Razani $^{1,2,3}$, Azhar Zam ${ }^{1,2,3}$, Nico J J Arezza ${ }^{1,2,3}$, Michael C Kolios ${ }^{1,2,3}$ \\ ${ }^{1}$ Department of Physics, Ryerson University, Toronto, Canada \\ ${ }^{2}$ Institute for Biomedical Engineering, Science and Technology (iBEST), a partnership between Ryerson Uni- \\ versity and St. Michael's Hospital, Toronto, Canada \\ ${ }^{3}$ Keenan Research Centre for Biomedical Science of St. Michael's Hospital, Toronto, Canada
}

\subsection{Abstract}

In this study, we present a technique to image the enhanced particle displacement generated using an acoustic radiation force (ARF) excitation source. A MEMS-VCSEL swept source Optical Coherence Tomography (SS-OCT) system with a center wavelength of 1310nm, a bandwidth of 100nm, and an A-scan rate of $100 \mathrm{kHz}$ was used to detect gold nanoparticle (70 $\mathrm{nm}$ in diameter) displacement. ARF was applied after the nanoparticles passed through a porous membrane and diffused into a collagen matrix (10\% collagen). B-mode, MB mode, 3D and Speckle Variance (SV) images were acquired before and after the ultrasound beam was on. Differential OCT speckle variance images with and without the ARF 
were used to estimate the particle displacement. The images were used to detect the microscopic enhancement of nanoparticle displacement generated by the ARF. Using this OCT imaging technique, the enhanced transport of particles though a porous membrane and a collagen gel after using an ARF excitation was imaged and analysed. In the vicinity of the focal point of the transducer generating the ARF, the enhancements were $62 \pm 5 \mu \mathrm{m}$ and $65 \pm 6.5 \mu \mathrm{m}$ in the axial and lateral directions, respectively. Using the SV-OCT technique, we are able to visualize and characterize the acoustic radiation force assisted enhanced displacement of gold nanoparticles (GNPs).

\subsection{Introduction}

The transport of nanoparticles through a leaky tumor vessel wall and into the interstitial space surrounding cancer cells, resulting in the accumulation of nanoparticles in the tumor, is called the enhanced permeability and retention effect. For this to occur, the nanoparticles must exit through the pores of the leaky tumor vessels while at the same time not passing through the pores of the normal vessels [1,2]. There are different type of nanoparticles which have been used as drug delivery vehicles such as polymeric micelles, liposomes, nanoemulsion droplets, metallic nanospheres, rods, shells and cages. Gold nanoparticles (GNPs) have attracted a great deal of attention as drug carriers since they can be imaged with multiple imaging modalities [3-6].

Particle size has a significant role in the therapeutic efficacy of the gold nanoparticle approaches due to the preferential cellular uptake of gold nanoparticles of particular sizes. Gold nanoparticles are typically in the size range of 2-100 $\mathrm{nm}$. It has been shown, in in-vitro 
studies, that particle sizes of 40-50 $\mathrm{nm}$ maximize intracellular uptake [7-9]. However, these nanoparticles have to also travel through the tumor extracellular matrix, which significantly affects the transport of the nanoparticles in-vivo [7]. This transport of nanoparticles through the extracellular matrix in turn determines the spatio-temporal distribution of the nanoparticles in the target tissue [8]. Characterization of the nanoparticle transport in tissue is important in assessing the effectiveness of this therapeutic approach. The collagen mesh associated with the tumor extracellular matrix can significantly inhibit nanoparticle diffusion / transport to the tumor cells [9]. Moreover, the increased pressure in the interstitial space due to poor lymphatic tumor drainage further inhibits nanoparticle penetration to the target cells [10]. Since such agents or drugs cannot come in close proximity to all the cancer cells, the treatment becomes less effective [11]. Techniques that can be used to enhance the particle delivery through the tumor extracellular matrix should in principle have an impact on the delivery of the nanoparticles to tumor cells. Imaging approaches in turn can be used to detect this enhanced transport and determine the effectiveness of the approaches used.

Ultrasound has been shown to enhance the delivery of therapeutic payloads to target tissues [1-4]. Physical parameters of the ultrasound beam (frequency, intensity and pulse duration) can be modified to introduce different mechanisms to achieve this effect $[5,6]$. One of the most effective approaches is to use the acoustic radiation force of a focused ultrasound beam to achieve such delivery [6]. Researchers have shown that such focused ultrasound exposures can be used to enhance the delivery of a variety of therapeutically important agents 
for the treatment of tumors and other diseases [21]. However, it is difficult to assess the efficacy of the ultrasound enhanced delivery in a real-time and non-invasive manner.

It is known that GNPs have a large light scattering cross-section, and therefore can be used as contrast agents in OCT [12]. In this study, we have developed a method to image the spatial distribution of the GNPs and therefore also assess the effect of introducing ARF to increase the penetration of GNPs in a collagen matrix. It is thought that this ARF approach can be used to partially overcome the problem of insufficient drug/agent delivery to the tumor.

Different imaging modalities such as ultrasound (US) imaging or magnetic resonance imaging (MRI) that can be used to visualize the extravasation of nanoparticles from leaky vessels and enhanced delivery of GNPs $[23,24]$. MRI and ultrasound have been used in the visualization and characterization of the extravasation process and the displacement of nanoparticles and for ultrasonically enhanced drug delivery. Drawbacks of MRI include cost, long clinical wait times, and technological complexity. Both US and MRI have spatial resolutions in the order of 0.1-1 mm, which is insufficient for detecting small and subtle changes in the spatial distribution of the GNP concentration, such as those that would be occurring in small tumors. Optical coherence tomography (OCT) is an optical tomographic imaging technique that shares many similarities to ultrasound imaging despite using light. OCT has several advantages over other imaging modalities, primarily due to its inherently high spatial and temporal resolution, which allows for the identification of micron sized morphological tissue structures and changes in these structures in time. We have developed a method using speck- 
le variance OCT and a focused ultrasound transducer for enhancing and monitoring GNPs based therapeutic delivery.

B-mode OCT can be used to image the spatial distribution of GNPs. The enhanced penetration of GNPs delivery using ARF is expected to be on the order of microns, depending on parameters related to the physical characteristics of the ultrasound beam and the viscoelastic/poroelastic properties of the tissues being exposed. Speckle variance OCT imaging has been shown to be sensitive to the microscopic motion of sub-resolution scatterers[25]. It is a decorrelation measurement method that enables visualization of slow movement flow as an independent of Doppler angle which was applied to high frequency ultrasound systems and Optical Coherence Tomography [25]. In this study, we have used speckle variance OCT imaging to analyze the enhanced delivery of GNPs.

\subsection{Method and materials}

A MEMS-VCSEL swept source OCT (Thorlabs, Inc.) system was used for this study. The laser has a center wavelength of 1310nm, a bandwidth of 100nm, and an A-scan rate of $100 \mathrm{kHz}$. The lateral and axial resolution were approximately $25 \mu \mathrm{m}$ and $12 \mu \mathrm{m}$ in the samples, respectively. The frame rate was $167 \mathrm{~Hz}$.

ARF (internal mechanical excitation) was applied after the particles were introduced into the collagen matrix and were allowed to reach a steady-state. B-mode images were acquired from the phantom. After diffusion, we acquired the BM-scans, 3D and Speckle Variance (SV) images, before and after the application of the acoustic radiation force. Differential 
OCT speckle variance images, with and without the ARF, were used to detect the particle displacement generated by the ARF.

Acoustic radiation force is produced by a change in the energy density of the incident acoustic field [26,27]. The acoustic radiation force is generated by the transfer of momentum from the acoustic wave to the tissue. The force is applied in the direction of the ultrasound wave propagation and the magnitude of the force can be approximated by Eq. (3.1):

$$
F=\frac{2 \alpha I}{C}
$$

Shear waves are also generated by using a focused impulse generated by an ultrasound transducer. The impulse creates a bulk tissue displacement in the direction of ultrasonic beam propagation, which is largest at the transducer focus. After the impulse, the material relaxes back to its original state producing a shear wave. The shear wave propagates in the direction perpendicular to the direction of the focused ultrasound propagation [28,29]. A similar acoustic radiation force is expected due to the momentum transfer of the propagating shear wave to the tissue.

To generate the ARF focussed impulse, a $20 \mathrm{MHz}$, circular, $8.5 \mathrm{~mm}$ radius piezoelectric transducer element (PZT, f-number 2.35, Visualsonics model \# 072-20-20) transmitting sine-wave bursts of $400 \mu$ s was used. Pulses sequences were designed to create periodic bursts of acoustic radiation force. Sine wave bursts of $400 \mu$ s were repeated every $16 \mathrm{~ms}$ for 
$5 \mathrm{~min}$ in total. The transducer focal depth was $20 \mathrm{~mm}$. The full width at half maximum of the beam at the focal point was calculated to be $246 \mu \mathrm{m}$.

Particle transport was examined in three stages. First the particle diffusion through a phantom was investigated with no radiation force applied (Fig.3.1a). The first phantom consisted of gelatin with 5\% concentration. Gelatin powder (Type B, Fisher Scientific, G7-500) and distilled water were heated in a water bath at $60-65^{\circ} \mathrm{C}$ for one hour and periodically stirred. When the sample cooled to $45^{\circ} \mathrm{C}$ then molten gelatin was poured into rectangle molds (20 mm height) and allowed to congeal. As the solution containing the GNPs was pumped through the tubing, we visualized the transport of the nanoparticles and detected an increase in the OCT backscatter from the nanoparticles diffusing outside of the tube. We used the tubing which had a single large opening (100-150 $\mu \mathrm{m})$ introduced to allow the particles to diffuse into the phantom matrix. Gelatin $(5 \%)$ was used to emulate a rudimentary extracellular matrix. The tube is connected to a syringe. Fig. 3.1(a) shows the schematic setup for this experiment.

In the second experiment, the sample consisted of $10 \%$ Collagen (Bovine Collagen Solution, Type I, Advanced BioMatrix). We used a porous membrane (Millicell Cell Culture Insert, $12 \mathrm{~mm}$, polycarbonate, $3.0 \mu \mathrm{m}$, EMD Millipore) which has multiple $3 \mu \mathrm{m}$ pores as a crude representation of the transport of GNPs through the leaky vasculature of a tumor. After the GNPs (70 nm in diameter, Nanocomposix Inc., San Diego, CA) were introduced into the collagen phantom and a quasi-steady-state was reached (minimal further transport of the gold nanoparticles through the collagen matrix), the experiments with the ARF enhancement were 
performed. At this last stage, the ultrasound transducer was turned on, which allowed to examine the effect of the ARF on the displacement of GNPs that already diffused through the $10 \%$ collagen. B-mode and B-M mode images were used for the analysis.

In order to measure the enhanced GNPs penetration through the collagen matrix that is caused by the ARF, the speckle variance technique was used [25]. The speckle variance (SV) was calculated using by Eq. (3.2):

$$
S V_{i j k}=\frac{1}{N} \sum_{i=1}^{N}\left(I_{i j k}-I_{\text {mean }}\right)^{2}
$$

where $\mathrm{N}$ is the number of the $\mathrm{B}$-frames and $\mathrm{j}$ and $\mathrm{k}$ are axial and transverse pixels in the image. In the results section, the number of frames is 6 and number of axial pixels and transverse pixels were 700 and 1024, respectively. Speckle variance maps of the phantom under ultrasound pulses were generated and the changes detected were related to the ARF 
induced displacement of the GNPs.

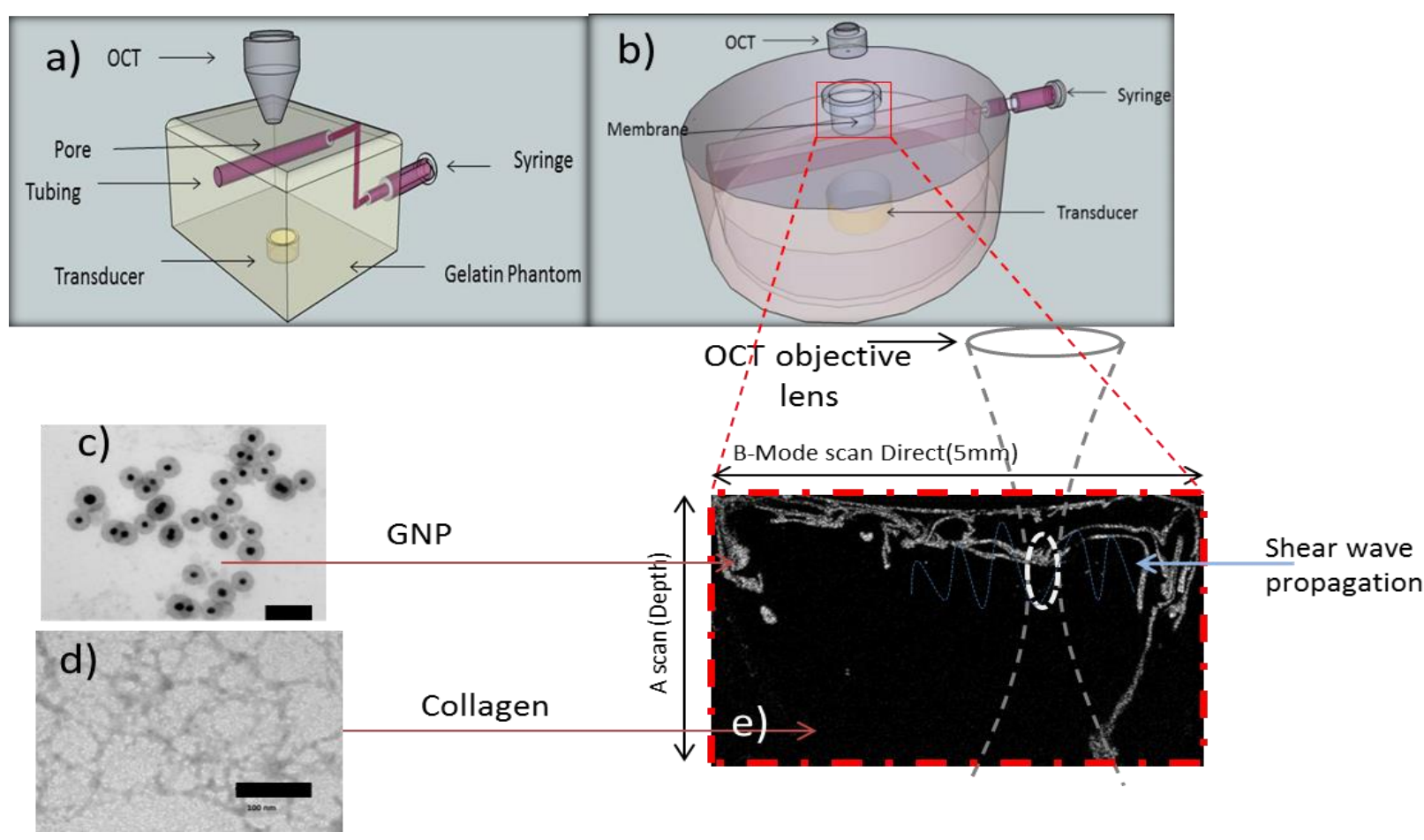

Figure 3-1. a) Schematic of the experiment for the tubing with a single opening (100-150 $\mu \mathrm{m})$ to allow for the diffusion of the GNPs out of the vessel, b) schematic of the experiment using the porous membrane ( $3 \mu \mathrm{m}$ pores). c) TEM image of GNPs d) TEM image of collagen matrix (scale bar is $100 \mathrm{~nm}$ ). e) Using an impulse generated by a focussed transducer, shear waves can be produced at the focal point that propagate radially outwards. The transducer focal depth for this study was $20 \mathrm{~mm}$. The shear wave generated travels through the collagen matrix also contributed to the displacement of the GNPs which diffused through the collagen. B-mode and SV OCT images were acquired at the focal point for SV analysis.

In order to calculate the enhanced displacement of the GNPs, 3D image volumes were obtained and then the speckle variance method was used for the analysis. The enhance transport of the GNPs can be measured by applying a simple algorithm to the speckle variance images as shown in Figure 3.2. The method relies on the subtraction of two speckle variance images as a method to track the movement of the unresolved GNPs clusters before and 
after exposure to the ARF. Even though a similar approach could be used based on OCT structural images, the speckle variance image has the advantage that it provides higher signal from the GNPs when the GNPs are situated in highly scattering environments (e.g. tissues, cells, etc.), due to the microscopic motion of the GNP clusters. The speckle variance images with the transducer on (which was on for 5 minutes) and then switched off were thresholded and binarized, and then subtracted to show the enhanced GNPs displacement in the axial and lateral directions. In the binarization process all speckle variance values were set to 1 and the background values to 0 . This allows the displacement in the axial and lateral direction to be better visualized in the speckle variance differential image. The differential speckle variance image (marked in red color in the speckle variance images) was then overlaid with the speckle variance image with the transducer off and a A scan profile at a particular location of interest was analysed to calculate the GNP cluster displacement in both the axial and lateral directions. 


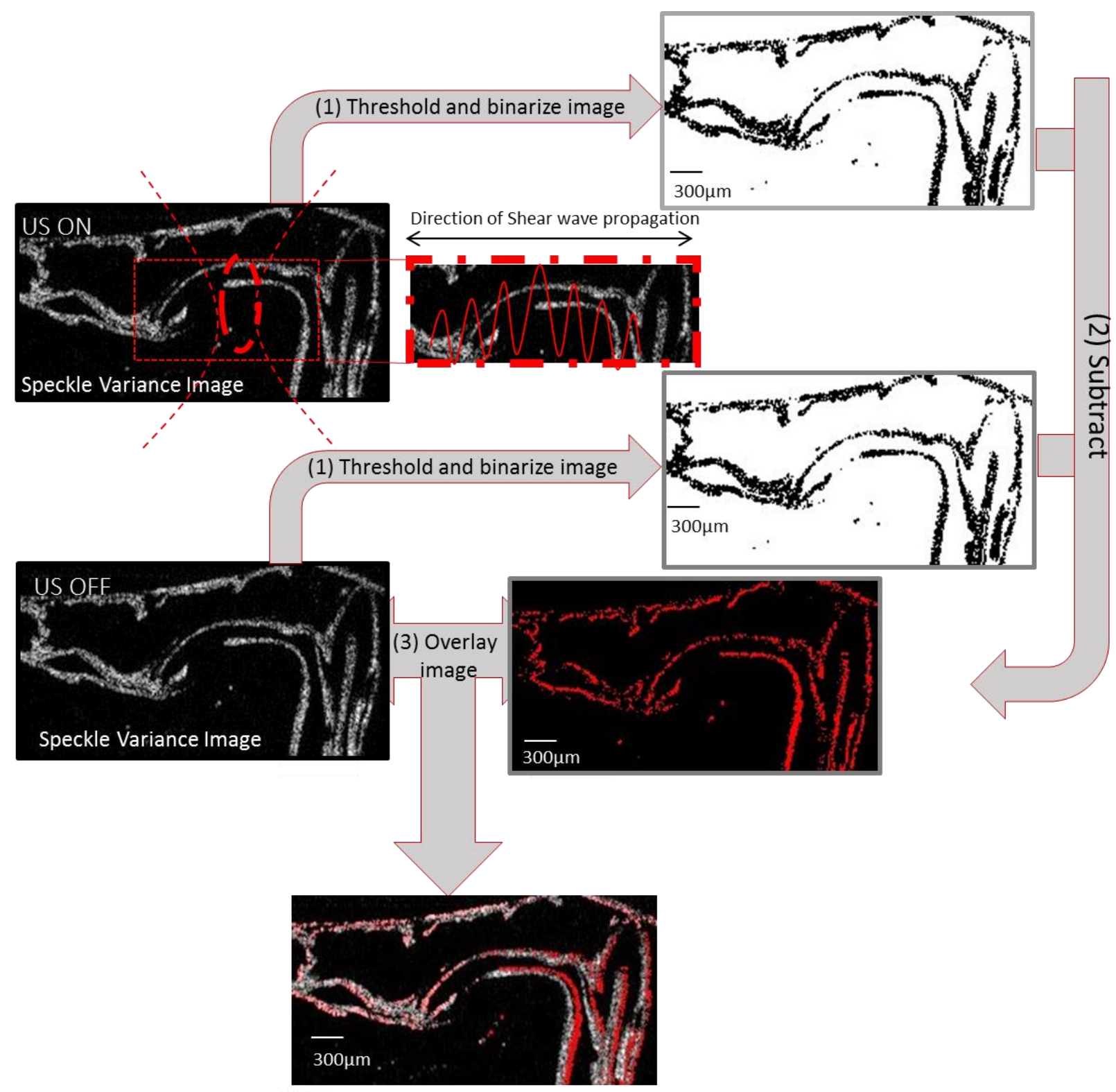

Figure 3-2. Processing steps of the algorithm for the measurement of the enhanced GNP delivery. The speckle variance images show the distribution of the GNPs in the collagen matrix. The speckle variance images with the transducer on (which was on for 5 minutes) and then switched off were thresholded and binarized, and then subtracted to show the enhanced GNPs displacement in the axial and lateral direction. The differential speckle variance image (marked in red color in the speckle variance images) was then overlaid with the speckle variance image with the transducer off. The red color pattern shows the differential speckle variance image when the ultrasound transducer is ON (US ON) and OFF (US OFF). 
The standard deviation of intensity was calculated by using Eq. (3.3):

$$
\operatorname{STD}_{i j k}=\frac{1}{N-1} \sum_{i=1}^{N} \sqrt{\left(I_{i j k}-I_{\text {mean }}\right)^{2}}
$$

Where $\mathrm{N}$ is number of $\mathrm{B}$ - Frames, $\mathrm{I}$ is intensity and $\mathrm{j}$ and $\mathrm{k}$ are axial and transverse pixels in the image. In this study, the standard deviation of intensity over $100 \mathrm{~B}$-frames (covering a time span of $600 \mathrm{~ms}$ ) was calculated.

To simulate the displacement of the collagen matrix in the presence of the acoustic radiation force, a finite element model was used. COMSOL (5.1, License number: 2076946) was used to simulate the propagation of shear waves through tissue and their effect on the medium displacement.

The model was developed using a 2D axisymmetric cylindrical geometry with elastic properties $(\mathrm{E}=27 \mathrm{kPa})$, that simulates a tissue equivalent phantom. A hemispherical transducer with similar characteristics to the experimental transducer (frequency $20 \mathrm{MHz}, 8.5 \mathrm{~mm}$ radius) was designed to generate the ultrasound waves. An acoustic intensity field was generated initially using the pressure acoustics physics interface in the Acoustics module. The wave equation solved is the homogeneous Helmholtz equation in 2D axisymmetric cylindrical coordinates.

After the intensity profile of the transducer was calculated, the total displacement of the tissue as a result of Acoustic Radiation Force excitation was solved and mapped as a function of time over an interval ranging from 0 to $6 \mathrm{~ms}$ with a step size of $0.1 \mathrm{~ms}$. The ultra- 
sound transducer was active in the interval ranging from $0.1 \mathrm{~ms}$ to $3.5 \mathrm{~ms}$ inclusive. The displacement and velocity fields that resulted from the acoustic radiation force were calculated over a time interval using the Solid Mechanics Physics from the Structural Mechanics module. The Solid Mechanics interface is based on solving the equations of motion together with a constitutive model for a solid material. Results such as displacements, stresses, and strains are computed. Two boundary conditions were used in this study: a perfectly matched layer when calculating the Acoustic Pressure and Low-Reflecting boundary during the time domain simulation that was used in the Solid Mechanics module. The Perfectly Matched Layer boundary conditions were used to prevent reflection of the waves back into the region of interest. 
Table 3.1: Parameters which used in the COMSOL simulation.

\begin{tabular}{llll}
\hline Parameter & Value & Unit & Description \\
\hline $\boldsymbol{\alpha}_{\text {phantom }}$ & 8.5 & $\mathrm{~m}^{-1}$ & Absorption coefficient of phantom \\
$\boldsymbol{\alpha}_{\text {water }}$ & 0.025 & $\mathrm{~m}^{-1}$ & Absorption coefficient of water \\
$\mathbf{B}$ & $4.497 \times 10^{6}$ & $\mathrm{~Pa}$ & Bulk modulus of phantom \\
$\mathbf{D}$ & 1000 & $\mathrm{~kg} / \mathrm{m}^{3}$ & Density of phantom \\
$\mathbf{E}$ & 27000 & $\mathrm{~Pa}$ & Young modulus of phantom \\
$\mathbf{f}$ & 20 & $\mathrm{MHz}$ & Ultrasound source frequency \\
$\mathbf{G}$ & 9006 & $\mathrm{~Pa}$ & Shear modulus of phantom \\
\hline $\boldsymbol{\mu}$ & 0.499 & $\mathrm{Dimensionless}$ & Poisson ratio of phantom \\
\hline
\end{tabular}

\subsection{Results}

Figure 3.3(a) shows the B mode video of the nanoparticles diffusing through the gelatin phantom. The particles were pumped through the tubing which was embedded in the gelatin phantom that had a single opening of approximately $100-150 \mu \mathrm{m}$. The diffusion of the GNPs could be visualized in the structural image. 


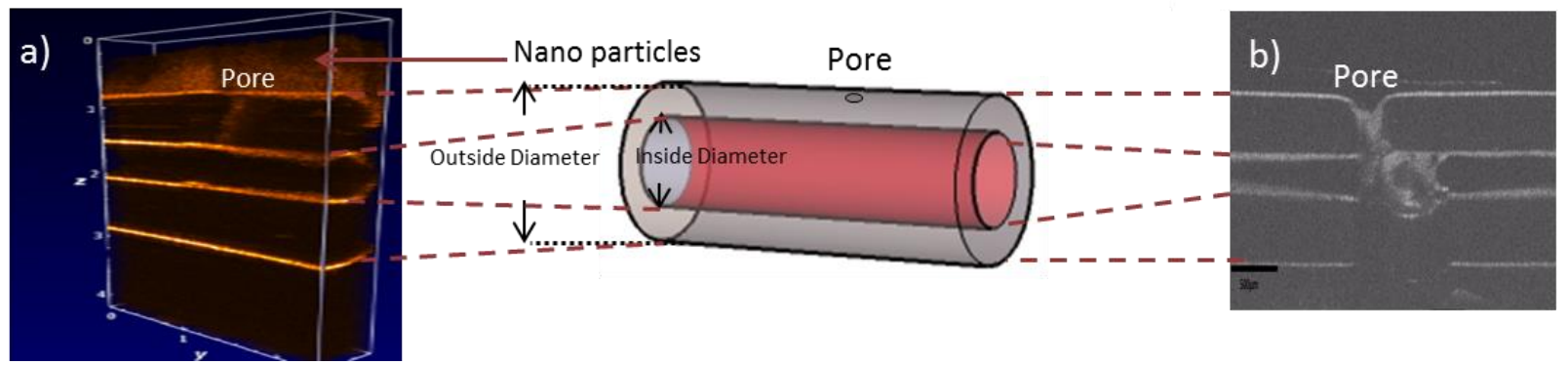

Figure 3-3 a) 3D OCT image of tubing after diffusion of GNPs though the opening b) B-mode OCT image of tubing which was embedded into the gelatin phantom and the nanoparticle distribution after the diffusion of the nanoparticles through the opening in the phantom (Media 1).

In the collagen matrix experiments, to demonstrate that a steady state was reached and the GNPs were not moving through the phantom, the speckle variance images of the phantom were obtained before the transducer was turned on (figure 3.4a). These images were acquired after a quasi-steady-state was reached (60 minutes after introduction of the GNPs). The pattern in the images is representative of the complex spatial distribution of the GNPs that is the result of the transport of the GNPs through the media and the complex interaction of the GNPs with the collagen matrix. Speckle variance images were taken when the ARF was off at $\mathrm{t}=0$ and at $\mathrm{t}=10$ seconds (this time interval was used to be consistent with the methodology used when the transducer was turned on). These images were then analyzed using the differential speckle variance technique and GNP displacement was calculated in both axial and lateral directions. There were no changes in the location of the peaks of the speckle variance images observed when the transducer was off (figure 3.4a). Another image as a negative control after 10 seconds was examined to show that the displacement measured in the subsequent experiments solely comes from the ARF (figure 3.4b). After the enhance- 
ment experiment detailed in the next paragraph was completed and the transducer was permanently turned off, speckle variance images were taken immediately after the transducer turned off $(t=0)$ and then 10 seconds after $(t=10 \mathrm{~s})$ while transducer remained off (figure 3.4b). Again, there were no changes observed in the two speckle variance images, indicating that the displacement induced by the ARF pulse sequence was permanent.

In the next set of experiments, the transducer was activated. Figure 3.5(a) shows the standard deviation of the intensity image of 100 OCT BM-scans acquired after transport of the GNPs through the collagen and after a quasi-steady-state was reached (60 minutes after injection). The scale bar represents the standard deviation. Images with the transducer on and off were acquired. Figure 3.5(b) shows speckle variance images of the phantom with the transducer on and off. By using this technique, we measured the aggregate displacement of the unresolved GNPs. The red pattern shows the differential speckle variance image. This pattern is overlaid on the speckle variance image before the transducer was turned on. After calculation from the regions of interest in figure3.5(b), the enhanced GNPs delivery measured was $62 \pm 5 \mu \mathrm{m}$ and $65 \pm 6.5 \mu \mathrm{m}$ at locations close to the focal point, in the axial and lateral directions respectively. To calculate the variability in the displacement measurements in regions adjacent to each other (and calculate a standard deviation related to this), three ROI(S) were selected close to the focal point area for each direction (lateral and axial directions). The three ROI(s) were 50 microns apart from each other. The displacement was calculated for each of these ROIs. From the three ROIs, the average and standard deviation in each direction were calculated. Speckle variance images were taken right away after we stopped the 
ARF $(t=0)$ and 10 seconds after $(t=10 s)$. There were no changes observed, again illustrating that the displacement measured was not transient and measured only when the transducer was on. 


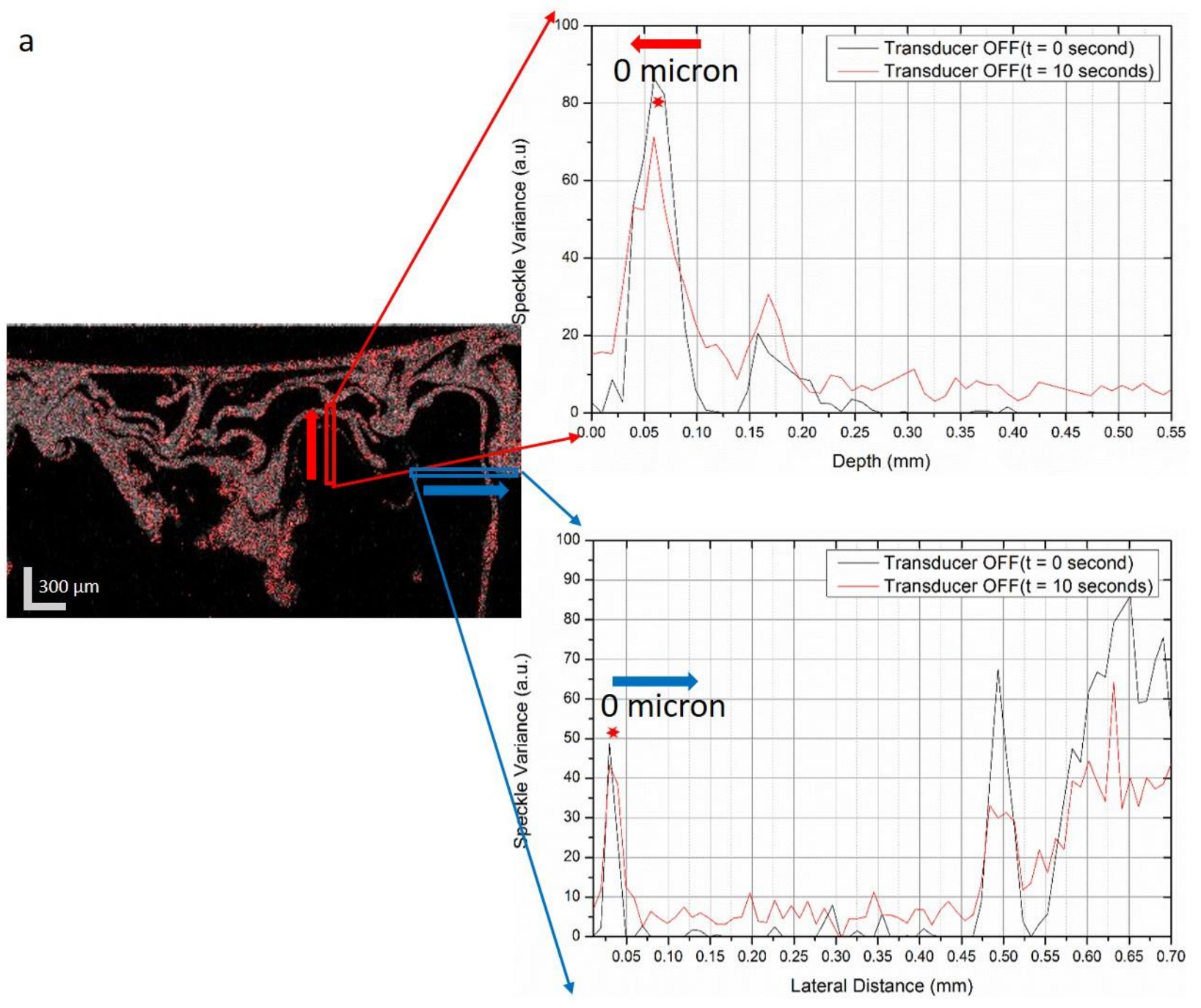




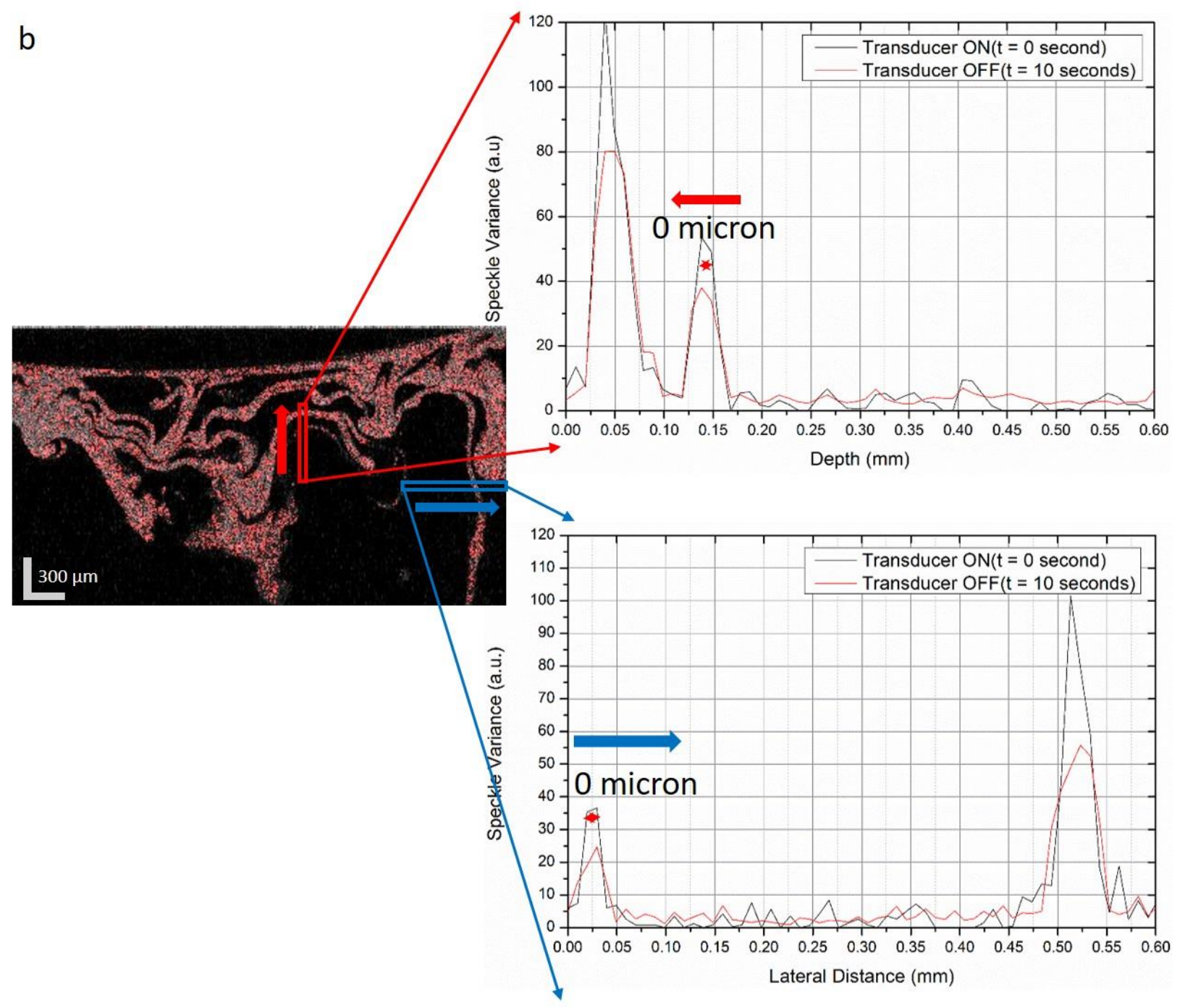

Figure 3-4 Speckle variance images of the phantom were obtained (a) when transducer was $\operatorname{OFF}(t=0)$ $\mathrm{OFF}(\mathrm{t}=10 \mathrm{~s})$.The pattern in the images is representative of the complex spatial distribution of the GNPs that is the result of the transport of the GNPs through the media and the complex interaction of the GNPs with the gel matrix. Speckle variance images were taken when $A R F$ was off at $t=0$ and at $t=10$ seconds (time interval was used to be consistent with the methodology used when the transducer was turned on). These images were then analyzed using the differential speckle variance technique and GNPs displacement was calculated in both axial and lateral directions. There were no changes in the location of the peaks of the speckle variance images observed when the transducer was off. (b) Another negative control after 10 seconds was examined to show that the displacement measured solely comes from the ARF when transducer $\mathrm{ON}(\mathrm{t}=0)-\mathrm{OFF}(\mathrm{t}=10 \mathrm{~s})$. These are negative control for the enhancement analysis. 
a
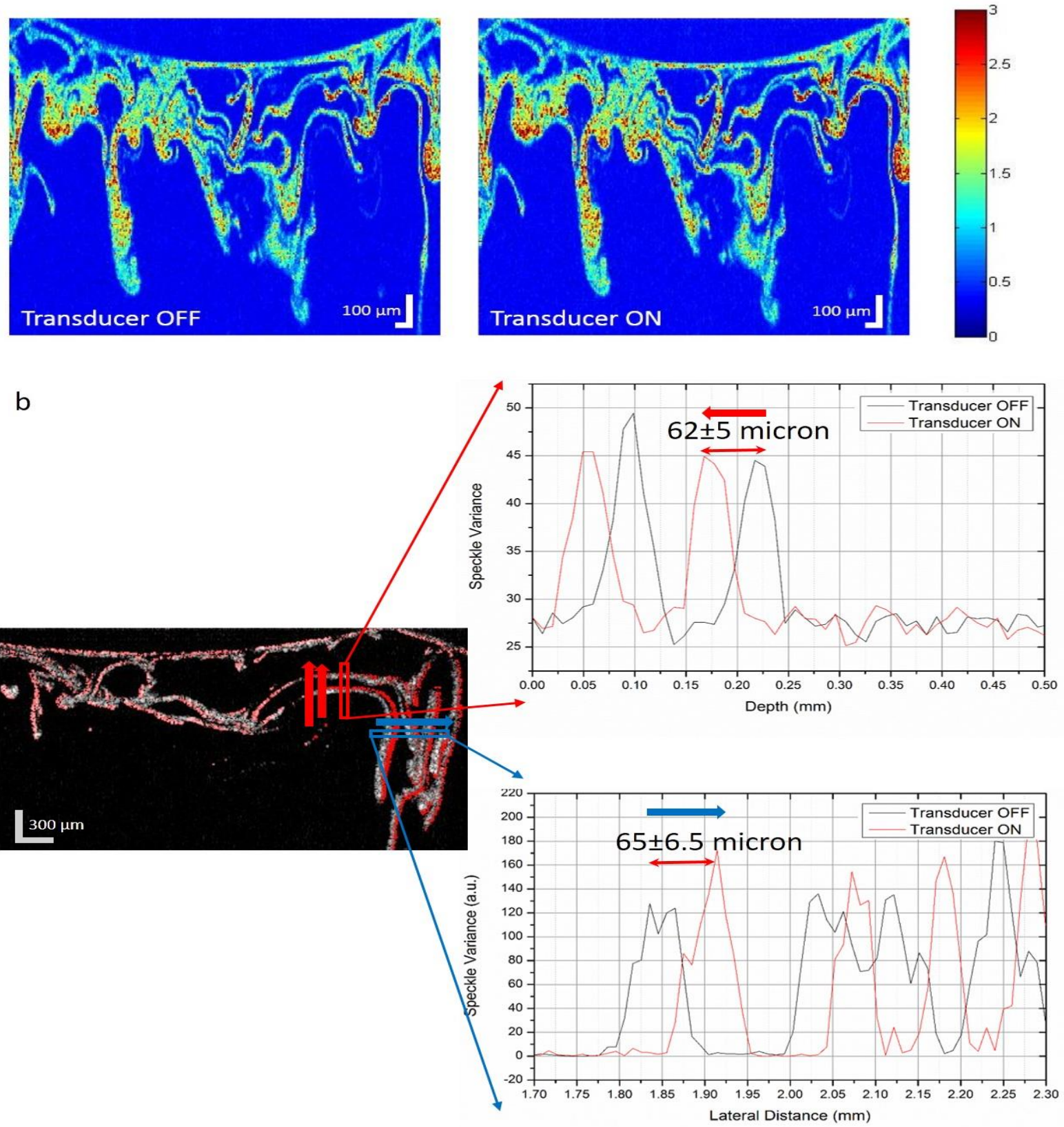

Figure 3-5 (a): 100 BM-scans OCT were acquired from GNPs through the collagen. The color map represents the standard deviation value (media2) (b): Speckle variance of after diffusion of GNPs through the collagen, and the calculation of enhanced delivery of the GNPs. The red and blue arrows denote the direction of the enhanced transport. The image (a) and (b) were acquired at different locations and time. 
In the simulations, the transient displacement of the tissue (the mesh in the simulations) as a result of the Acoustic Radiation Force excitation was calculated as a function of time. The simulation in this study was compared and validated well to published displacement values using other Finite element methods (FEM) [21]. Figure 3.6 shows the simulated displacement of the solid which was induced using the ARF from a $20 \mathrm{MHz}$ transducer within an isotropic linear elastic solid in the COMSOL simulation. The displacement fields of the mesh were shown at time of $0,1.7,2.8,3.8$ and $4.8 \mathrm{~ms}$. In these images, the color bar represents the displacement (in $\mu \mathrm{m}$ ). From these fields, displacement over time in the plot and is shown in figure 3.7. The greatest displacement occurred in the focal region, as expected. The displacements of the mesh calculated are of the same order of magnitude as the measured GNP displacements in the experiments described above. 

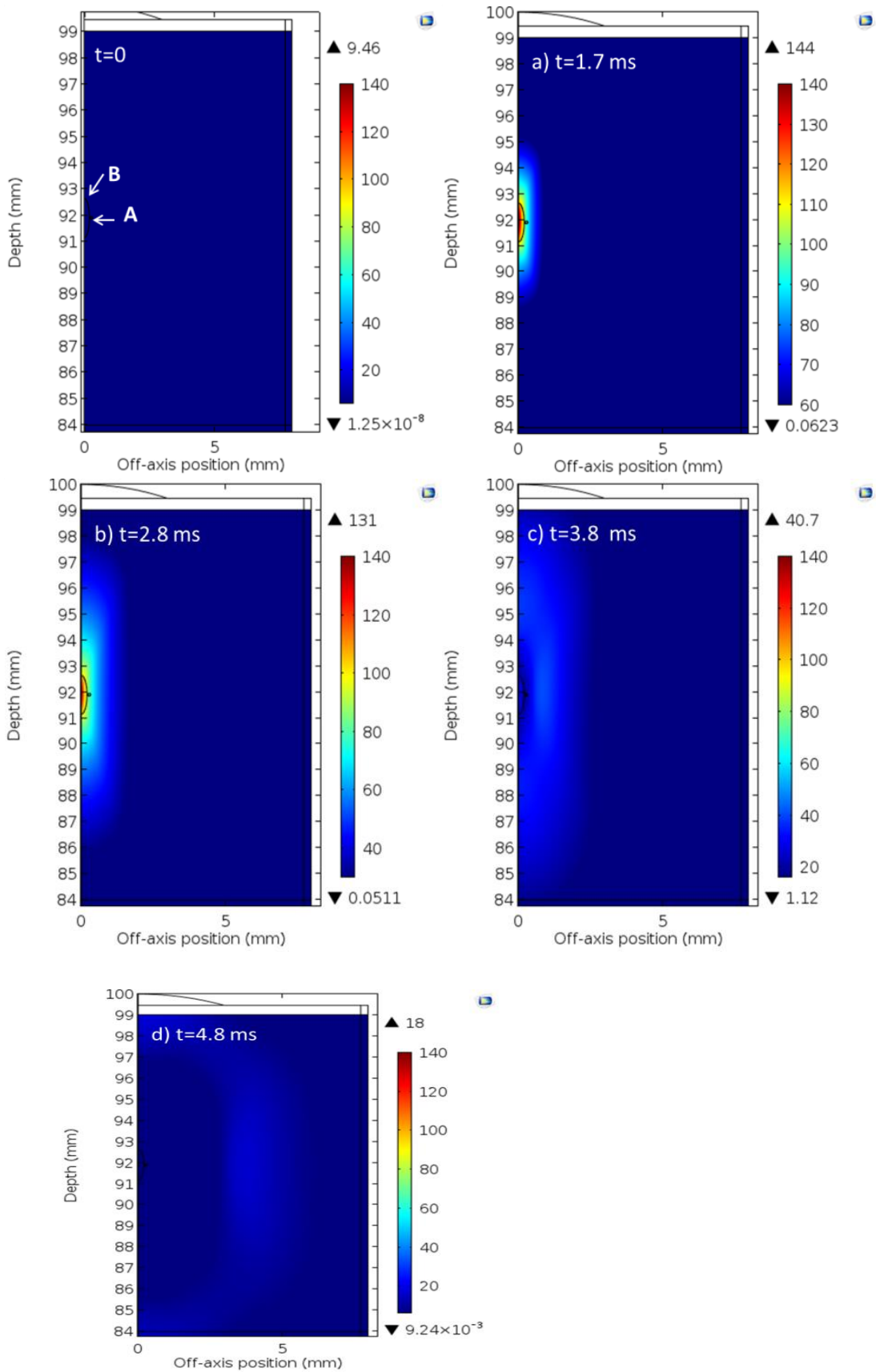

Figure 3-6ARF-induced displacement in phantom. The plots depict the displacement of the phantom mesh at various points in time as a result of ARF excitation and the color bar represents displacement in microns. The displacement fields were taken at times $0 \mathrm{~ms}, 1.7 \mathrm{~ms}$ (a), $2.8 \mathrm{~ms}$ (b), $3.8 \mathrm{~ms}$ (c), and $4.8 \mathrm{~ms}$ (d) respectively. 


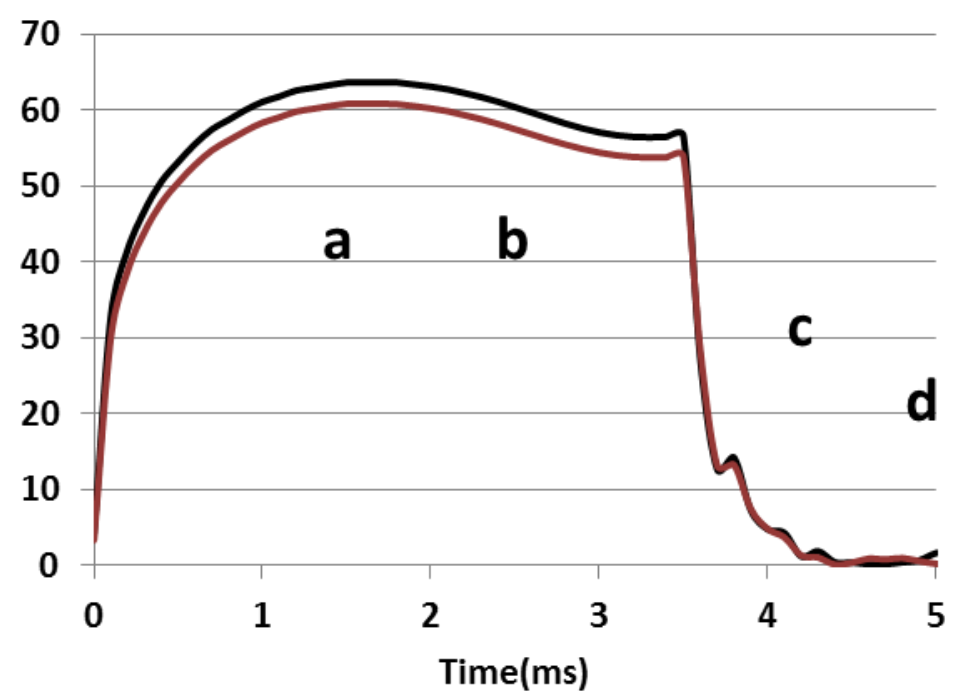

Figure 3-7 Simulated displacement of two points in the phantom which was induced using the ARF from a $20 \mathrm{MHz}$ transducer, within an isotropic, linear elastic solid and points of a,b,c and d depict to the displacement point at time $1.7 \mathrm{~ms} \mathrm{(a),} 2.8 \mathrm{~ms}$ (b), $3.8 \mathrm{~ms}$ (c), and $4.7 \mathrm{~ms}$ (d) respectively. The black line represents the displacement in the vicinity of the focal point $(\mathrm{A})$ and red line represents the displacement above this point (B) (see figure3. 6). The two points are $250 \mu \mathrm{m}$ apart.

\subsection{Discussions}

In summary, shear waves were generated in an extracellular matrix phantom by using acoustic radiation force. The acoustic radiation force generated by both longitudinal waves and shear waves increased the displacement of the GNPs through the phantom. In this study, gold nanoparticle (70 $\mathrm{nm}$ in diameter) and collagen $10 \%$ used . After applying ARF, the enhancements were $62 \pm 5 \mu \mathrm{m}$ and $65 \pm 6.5 \mu \mathrm{m}$ in the axial and lateral directions, respectively. We have shown that the ARF can be used to enhance the transport of GNPs within a collagen matrix. By using the ARF-OCT technique, we are able to visualize and characterize of the acoustic radiation force assisted enhancement of delivery gold nanoparticles using an OCT 
technique. Also, though ARF has been simulated in past studies using FEM methods [21], COMSOL allows the coupling of the results of wave propagation to simulate other phenomena such as enhanced diffusion, which will be studied in future work. The experimental results are consistent with simulation results which show that the displacement of the mesh induced by the ARF is of the same order of magnitude as the enhanced transport of the GNPs as measured with the OCT technique presented in this work. This study demonstrated the feasibility of using speckle variance OCT to measure the enhanced delivery of GNPs after using acoustic radiation force. We have developed a method using ARF-OCT for enhancing and monitoring GNP based therapeutic delivery.

We demonstrated the feasibility for SV-OCT to measure the enhancement of nanoparticles in the sample as a stepping stone for expanding the research into observing enhancement of delivery drug into the tumour cells.

\section{Acknowledgments}

Marjan Razani is supported by Natural Sciences and Engineering Research Council of Canada (NSERC) Postgraduate Doctoral Scholarship (PGSD3).

This work is funded in part by the Natural Sciences and Engineering Research Council of Canada (NSERC discovery grant 216986-07) and Canada Foundation for Innovation (awarded to Dr. M. C. Kolios). We would like to thank Mr. Yaraskavitch for his contributions, Dr. Daghighi and Dr. Palmeri for their discussions. 


\section{References}

1. Triantafyllos Stylianopoulos, Rakesh K. Jain " Design considerations for nanotherapeutics in oncology" Nanomedicine, 11:1893-1907 (2015).

2. Sarin H, "Physiologic upper limits of pore size of different blood capillary types and another perspective on the dual pore theory of microvascular permeability",J.Angiogenes Res.;2:14. (2010).

3. N Rapoport "Phase-shift, stimuli-responsive perfluorocarbon nanodroplets for drug delivery to cancer”.Wiley Interdiscip Rev Nanomed Nanobiotechnol;4:492-510(2012).

4. Yohan, D. and B.D. Chithrani, "Applications of nanoparticles in nanomedicine". Journal of Biomedical Nanotechnology, 10(9): p. 2371-2392(2014).

5. Yang, C., et al., "Cancer nanotechnology: Enhanced therapeutic response using peptidemodified gold nanoparticles". Journal of Nanoscience and Nanotechnology, 14(7): p. 48134819(2014).

6. S. Zackrisson, S. M. van de Ven, and S. S. Gambhir, B "Light in and sound out: Emerging translational strategies for photoacoustic imaging”, Cancer Res., vol. 74, no. 4, pp. 9791004,(2014).

7. Wim H De Jong and Paul JA Borm "Drug delivery and nanoparticles: Applications and hazards", Int J Nanomedicine. 3(2): 133-149 (2008).

8. Jiang W, Kim BY, Rutka JT, Chan WC. Nanoparticle-mediated cellular response is sizedependent. Nat Nanotechnol;3:145-50 (2008).

9. Chithrani, B.D., A.A. Ghazani, and W.C. Chan, "Determining the size and shape dependence of gold nanoparticle uptake into mammalian cells”,. Nano Lett,. 6(4): p. 662-8. (2006).

10. Jain, R.K., J.D. Martin, and T. Stylianopoulos, The role of mechanical forces in tumor growth and therapy. Annu Rev Biomed Eng, 16: p. 321-46(2014.).

11. Huang, H. C.; Rege, K.; Heys, J. J. "Spatiotemporal Temperature Distribution and Cancer Cell Death in Response to Extracellular Hyperthermia Induced by Gold Nanorods", ACS Nano 4, 2892-2900(2010).

12. Minchinton, A.I. and I.F. Tannock, "Drug penetration in solid tumours". Nature Reviews Cancer,. 6(8): p. 583-592(2006). 
13. Stylianopoulos, T., et al., "Coevolution of solid stress and interstitial fluid pressure in tumors during progression: implications for vascular collapse”,. Cancer Res,. 73(13): p. 383341(2013).

14. Trédan, O., et al., "Drug resistance and the solid tumor microenvironment". Journal of the National Cancer Institute,. 99(19): p. 1441-1454(2007).

15. Mitragotri, S., D. Blankschtein, and R. Langer, "Ultrasound-mediated transdermal protein delivery”,. Science,. 269(5225): p. 850-853(1995).

16. Mitragotri, S., "Healing sound: The use of ultrasound in drug delivery and other therapeutic applications". Nature Reviews Drug Discovery, 4(3): p. 255-260(2005).

17. Pitt, W.G., G. Husseini, and B.J. Staples, "Ultrasonic drug delivery - A general review". Expert Opinion on Drug Delivery,. 1(1): p. 37-56(2004).

18. Hancock, H.A., et al., "Investigations into Pulsed High-Intensity Focused UltrasoundEnhanced Delivery: Preliminary Evidence for a Novel Mechanism”. Ultrasound in Medicine and Biology,. 35(10): p. 1722-1736(2009).

19. Dalecki, D., "Mechanical bioeffects of ultrasound", in Annual Review of Biomedical Engineering.. p. 229-248(2004).

20. Dayton, P., et al., "Acoustic radiation force in vivo: A mechanism to assist targeting of microbubbles". Ultrasound in Medicine and Biology,. 25(8): p. 1195-1201(1999).

21. Hilary A. Hancock, Lauren H. Smith, Julian Cuesta, Amir K. Durrani, Mary Angstadt, Mark L. Palmeri, Eitan Kimmel, and Victor Frenkel "Investigations into pulsed-high intensity focused ultrasound enhanced delivery: Preliminary evidence for a novel mechanism” Ultrasound Med Biol. 35(10): 1722-1736(2009).

22. Troutman, T.S., J.K. Barton, and M. Romanowski, Optical coherence tomography with plasmon resonant nanorods of gold. Optics Letters,. 32(11): p. 1438-1440(2007).

23. Tatsuya Nakamura, Fuminori Sugihara, Hisashi Matsushita, Yoshichika Yoshioka, Shin Mizukami and Kazuya Kikuchi, "Mesoporous silica nanoparticles for magnetic resonance imaging, fluorescence imaging, and drug delivery”, Chem. Sci ,6, 1986-1990(2015).

24. Paul A. Dayton, Shukui Zhao,Susannah H. Bloch, Pat Schumann,Kim Penrose, Terry O. Matsunaga, Reena Zutshi, Alexander Doinikov, and Katherine W. Ferrara “Application of Ultrasound to Selectively Localize Nanodroplets for Targeted Imaging and Therapy”, Mol Imaging. 5(3): 160-174(2006). 
25. A. Mariampillai, B. A. Standish, E. H. Moriyama, M. Khurana, N. R. Munce, M. K. K. Leung, J. Jiang, A. Cable, B. C. Wilson, I. A. Vitkin, and V. X. D. Yang, "Speckle variance detection of microvasculature using swept-source optical coherence tomography," Opt. Lett. 33(13), 1530-1532 (2008).

26. J. McLaughlin, and D. Renzi, "Using level set based inversion of arrival times to recover shear wave speed in transient elastography and supersonic imaging," Inverse Probl, 22, 707725 (2006).

27. M. L. Palmeri, S. A. McAleavey, G. E. Trahey, and K. R. Nightingale, "Ultrasonic tracking of acoustic radiation force-induced displacements in homogeneous media," IEEE Trans. Ultrason., Ferroelectr., Freq. Control, 53(7), 1300-1313 (2006).

28. M.Razani, A. Mariampillai, C.Sun, T WH Luk, V XD Yang, M C Kolios” Feasibility of optical coherence elastography measurements of shear wave propagation in homogeneous tissue equivalent phantoms" Biomed. Opt. Exp., vol. 3, no. 5, pp. 972-980( 2012).

29. M. Razani, T. W. H. Luk, A. Mariampillai, P. Siegler, T.-R. Kiehl, M. C. Kolios, and V. X. D. Yang, "Optical coherence tomography detection of shear wave propagation in inhomogeneous tissue equivalent phantoms and ex-vivo carotid artery samples,” Biomed. Opt. Express 5(3), 895-906 (2014). 


\title{
Chapter 4: Acoustic radiation force en- hanced displacement of gold nanoparticles (GNPs) monitored by using OCT
}

\author{
Authors: Marjan Razani, Azhar Zam, Michael C Kolios \\ Marjan Razani ${ }^{1,2,3}$, Azhar Zam ${ }^{1,2,3}$, Michael C Kolios ${ }^{1,2,3}$ \\ ${ }^{1}$ Department of Physics, Ryerson University, Toronto, Canada \\ ${ }^{2}$ Institute for Biomedical Engineering, Science and Technology (iBEST), a partnership between Ryerson Uni- \\ versity and St. Michael's Hospital, Toronto, Canada \\ ${ }^{3}$ Keenan Research Centre for Biomedical Science of St. Michael's Hospital, Toronto, Canada
}

\subsection{Abstract}

In this study, an acoustic radiation force (ARF) excitation source was used to enhance the transport of gold nanoparticles (GNPs) in a collagen matrix. A swept source Optical Coherence Tomography (SS-OCT) system with a center wavelength of $1310 \mathrm{~nm}$ and an A-scan rate of $100 \mathrm{kHz}$ was used to detect gold nanoparticle (70 nm in diameter) displacement. ARF was applied after nanoparticle injection and diffusion/transport into a collagen matrix, with and without the presence of cancer cells. Different concentrations of collagen (3\% and 6\%) and a tissue engineered cellular construct based on MCF7 breast cancer cells embedded in a collagen matrix were used in this study. B-mode, M-B mode, 3D and Speckle Variance (SV) images were acquired before and after the ultrasound ARF was applied. Differential OCT speckle variance images, with and without the ARF, were used to calculate the GNP dis- 
placement. Enhanced displacement of the GNPs was calculated in both the axial and lateral directions at locations close to the ultrasound focal point, associated with longitudinal waves and shear waves, respectively. In the vicinity of the focal point of the transducer generating the ARF, enhanced displacements were calculated of $95 \pm 8 \mu \mathrm{m}$ for $6 \%$ collagen, $170 \pm 8 \mu \mathrm{m}$ for $3 \%$ collagen and $21 \pm 1 \mu \mathrm{m}$ for the MCF7 module in the lateral direction. The displacement associated with the enhanced transport decreased as the collagen concentration increased. Using the SV-OCT technique, we are able to visualize and characterize the acoustic radiation force assisted enhanced displacement of gold nanoparticles (GNPs) in collagen matrices. 


\subsection{Introduction}

A significant impediment to the use of nanoparticles (NPs) for cancer treatment is the transport of the nanoparticles throughout the tumor [1]. Nanoparticle therapy is dependent on nanoparticles reaching the target cancer cells to deliver the therapeutic effect $[2,3]$. If NPs are injected systemically into the vasculature, the NPs have to first enter into the tumor interstitium by exiting the tumor vessels [4-6]. To achieve a therapeutic advantage, the particles have to accumulate in the tumor tissue preferentially compared to the normal tissue. This can be achieved through the enhanced permeability and retention effect (EPR) [7-9]. Even after exiting the tumor vasculature, the NPs have to travel through the tumor stroma to expose all tumor cells to the NPs, in order to maximize therapeutic effect. The penetration of the nanoparticles throughout the tumor tissue is complex and depends on many factors $[10,11]$. One such factor is the high collagen content in a tumor's extracellular matrix and the formation of a thick fibrous network which is dominant in particularly poorly vascularized and hypoxic regions. This increases tissue stiffness and generates a barrier that limits the penetration of NPs. High collagen content fiber regions will impede NP diffusion by steric hindrance and high localized viscosity. Consequently, it is important to ensure that the transport of the NPs through the tissues is monitored to ensure adequate NP delivery. Gold nanoparticles specifically have attracted scientific and technological interest because of their chemical stability and unique optical properties [12]. Gold nanoparticles $\left(\mathrm{GNP}_{\mathrm{S}}\right)$ with diameter less than $100 \mathrm{~nm}$ also have practical applications in optics in nanomedicine with ab- 
sorbance peaks between 520nm-550nm that enable imaging and therapeutic applications [1323].

Facilitating NP transport through the tumor interstitial space is a challenge [24], as NP delivery depends on factors such as the distances between tumor blood vessels (which in tumors are immature and chaotically branched), adhesion between tumor cells, the extracellular matrix, high interstitial fluid pressure, the lack of convection and binding of the NPs to cells and extracellular structures [25]. Therefore, different approaches have been used to enhance the penetration of drugs or NPs through the tumor tissue. Ultrasound has been used as a method to enhance drug delivery [26]. Ultrasound creates rapid changes in localized pressure fields which create confined convection currents and shear stress on cells and tissue constituents, especially in the presence of ultrasound contrast agents (gas filled microbubbles). Focussed ultrasound has shown considerable promise as an approach to enhance payload delivery due to the localized and targeted delivery of the US energy [27,28]. For example, pulsed high intensity focused ultrasound was found to increase penetration and therapeutic efficacy of monoclonal antibodies in murine tumors [29].

Acoustic radiation force is produced by a change in the energy density of the incident acoustic field $[30,31]$. The transfer of momentum from the acoustic wave to the tissue equivalent phantoms results in the acoustic radiation force. This force is applied in the direction of the ultrasound wave propagation and the magnitude of the acoustic radiation force can be approximated by Eq. (4.1):

$$
F=\frac{2 \alpha I}{C}
$$


where $\mathrm{C}(\mathrm{m} / \mathrm{s})$ is the speed of sound in the medium, $\mathrm{F}\left(\mathrm{kg} \mathrm{s}^{-2} \mathrm{~cm}^{-2)}\right.$ is the acoustic radiation force, $\alpha(\mathrm{Np} / \mathrm{m})$ is the absorption coefficient of the medium and $\mathrm{I}\left(\mathrm{W} / \mathrm{cm}^{2}\right)$ is the temporal average intensity at a given spatial location [32]. When the acoustic radiation force is applied to a given spatial volume for a short time duration, shear waves are generated that propagate away from the initial region of excitation (i.e. the focal spot region) and parallel to the transducer [33]. Shear wave propagation speed and attenuation are directly related to the mechanical properties of the tissue. Due to the high attenuation of shear waves, it is possible to generate the shear wave within a very limited area of tissue [33]. The spatial frequency content of the shear wave will be determined primarily be the width of the ultrasound beam (and not the time duration of the excitation unless the excitation duration approaches the natural time constants of soft tissues). This is turn dictates the frequency of the shear wave produced; smaller ARF beams produce higher frequency shear waves [34, 35]. These shear waves in turn produce an acoustic radiation force that is related to the change in the energy density of the propagating shear wave.

There are several advantages with the use of focused ultrasound excitation as a mechanical wave source for facilitating NP delivery. Advantages include the non-invasive nature of ARF excitation to induce deformations in depth in tissue and the control of the ultrasound transducer geometrical characteristics and frequency. This control allows the creation of a wide variety of longitudinal and shear waves (with different frequencies) that irradiate the tissues at different spatial and temporal scales, which would be much more difficult to achieve using other methods (such as a laser pulse). Moreover, the formation of shear waves 
at the ultrasound focus is anticipated to create an acoustic radiation force perpendicular to the beam, further displacing particles in those directions [36,37].

Since monitoring the distribution of NPs in tissue is important, various techniques have been used to monitor the spatial distribution of the NPs non-invasively. Two-Photon Luminescence, Confocal Microscopy [38,39] and Optical Coherence Tomography are several imaging techniques that have been used to detect NPs in tissue. Optical Coherence Tomography $(\mathrm{OCT})$ is an optical tomographic imaging technique that is very similar to ultrasound imaging despite using light. OCT has high spatial and temporal resolution, which enable the characterization of micron sized morphological tissue structures and dynamic changes in the tissue structure. This higher resolution comes at the expense of limited imaging penetration depth, which is on the order of a few millimeters. Therefore, most clinical applications are limited to superficial tissues or to imaging on an interstitial / intravascular probe. The technology is also flexible and inexpensive [40]. There are resolution and sensitivity advantages when using ARF excitation and OCT for enhancement detection. Speckle variance optical coherence tomography (SV-OCT) has become a significant imaging modality useful in the 3D non-invasive imaging of in vivo microvasculature [41]. Speckle variance OCT is a measurement technique that relies on the decorrelation of the OCT signal and enables visualization of slow moving scatterers, independent of Doppler angle. We have recently used this approach to examine the microscopic movement of NPs, and their net displacement after ultrasound excitation [42]. The high spatial resolution of OCT allows for the detection of the 
small enhancements in NP penetration in the samples that are important to the assessment of the enhanced NP delivery.

We present the OCT measurements of the nanoparticle enhanced displacements in the samples as a stepping stone for expanding the research into observing enchantment of delivery into tumours, in-vivo. To achieve this, we measured the enhanced GNPs displacement achieved using the ARF excitation after the injection of the GNPs into collagen phantoms with two different collagen concentrations: $3 \%$ and $6 \%$. The same ARF excitation was used to enhance the displacement of injected GNPs in a tissue engineering module, which contained MCF-7 breast cancer cells, that more closely emulates tumor tissue.

\subsection{Methods}

In this study, the samples imaged in the first step of experiments consisted of collagen gels using 3\% and 6\% collagen (Bovine Collagen Solution, Type I, Advanced BioMatrix, San Diego). In the 3D gel preparation procedure, collagen was slowly mixed with 10X PBS and the $\mathrm{pH}$ of the mixture was adjusted to $7.2-7.6$ by using sterile $0.1 \mathrm{M} \mathrm{NaOH}$. The mixture was then warmed to $37^{\circ} \mathrm{C}$. We allowed approximately 90 to 120 minutes for gel formation.

In the second experiment, tissue engineered collagen modules were seeded with MCF7 breast cancer cells. Collagen modules were prepared as previously described [43]. In brief, collagen at initial concentration 0.3\% (Bovine Collagen Solution, Type I, Advanced BioMatrix, San Diego) used and was mixed with 10x minimum essential media, and neutralized with $0.8 \mathrm{M} \mathrm{NaHCO}$ (60 $\mu$ Lof $10 \times$ medium per ml of collagen) and kept on ice. MCF7 cells, grown in DMEM (Gibco) supplemented with 10\% fetal bovine serum (FBS) and $0.1 \%$ 
insulin at $37^{\circ} \mathrm{C}$ and $5 \% \mathrm{CO} 2$, then were trypsinized, counted, and then resuspended at $4 \mathrm{x}$ $10^{6} / \mathrm{mL}$ ( in the collagen solution. The modules were centrifuged for $\sim 5 \mathrm{~min}$ at $125 \mathrm{~g}$ to form a pellet. The majority of the supernatant was removed, and the modules were transferred to the gel for OCT imaging and measure GNP transport through the MCF7 module. Three independent experiments were performed.

The $\mathrm{GNP}_{\mathrm{S}}$ were injected into the collagen $(1 \mathrm{mg} / \mathrm{mL}$ gold concentration). After the GNPs were introduced into the collagen phantoms (70 $\mathrm{nm}$ in diameter, Nanocomposix Inc., San Diego, CA) and a quasi-steady-state was reached 60 minutes after introduction of the GNPs, there was minimal further transport of the GNPs through the collagen matrix. The experiments with the ARF enhancement were performed at this time. B-mode and B-M mode images were used for the analysis. A MEMS-VCSEL swept source OCT system (Thorlabs, Inc.) was used for imaging the collagen samples. The system had a laser a center wavelength of $1310 \mathrm{~nm}$, a bandwidth of $100 \mathrm{~nm}$, an A-scan rate of $100 \mathrm{kHz}$ with a lateral and axial resolution of approximately $25 \mu \mathrm{m}$ and $12 \mu \mathrm{m}$ in the imaged samples, respectively. ARF (internal mechanical excitation) was applied after the NPs were injected into the different collagen matrix concentrations and the MCF7 tissue engineered modules. Following the diffusion of the GNPs, B-mode images were acquired from the samples. Next, we performed BM-scans, 3D and Speckle Variance (SV) images, prior to and after the application of the acoustic radiation force. Differential OCT speckle variance images, with and without the ARF, were used to detect the particle displacement generated by the ARF. 
To generate the ARF focussed impulse, a $20 \mathrm{MHz}$, circular, $8.5 \mathrm{~mm}$ radius piezoelectric transducer element (PZT, f-number 2.35) transmitting sine-wave bursts of $400 \mu$ s was used. Pulses sequences were designed to create periodic bursts of acoustic radiation force. Sine wave bursts of $400 \mu$ s were repeated every $16 \mathrm{~ms}$ for $5 \mathrm{~min}$ in total. The transducer focal depth was $20 \mathrm{~mm}$. The full width at half maximum of the beam at the focal point was calculated to be $246 \mu \mathrm{m}$.

In order to measure the enhanced $\mathrm{GNP}_{\mathrm{s}}$ penetration through the collagen matrix that is caused by the ARF, the speckle variance technique was used [41]. The speckle variance (SV) in the OCT image was calculated using by Eq. (4.2):

$$
S V_{i j k}=\frac{1}{N} \sum_{i=1}^{N}\left(I_{i j k}-I_{\text {mean }}\right)^{2}
$$

where $\mathrm{N}$ is the number of the $\mathrm{B}$-frames and $\mathrm{j}$ and $\mathrm{k}$ are axial and transverse pixels in the image. For the results presented, the number of frames is 6 and number of axial pixels and transverse pixels were 700 and 1024, respectively. Speckle variance maps of the phantom were generated and changes detected were related to the ARF induced displacement of the GNPs. Figure 4.1 shows a schematic diagram of Acoustic Radiation Force-Optical Coherence Tomography (ARF-OCT) set-up. 

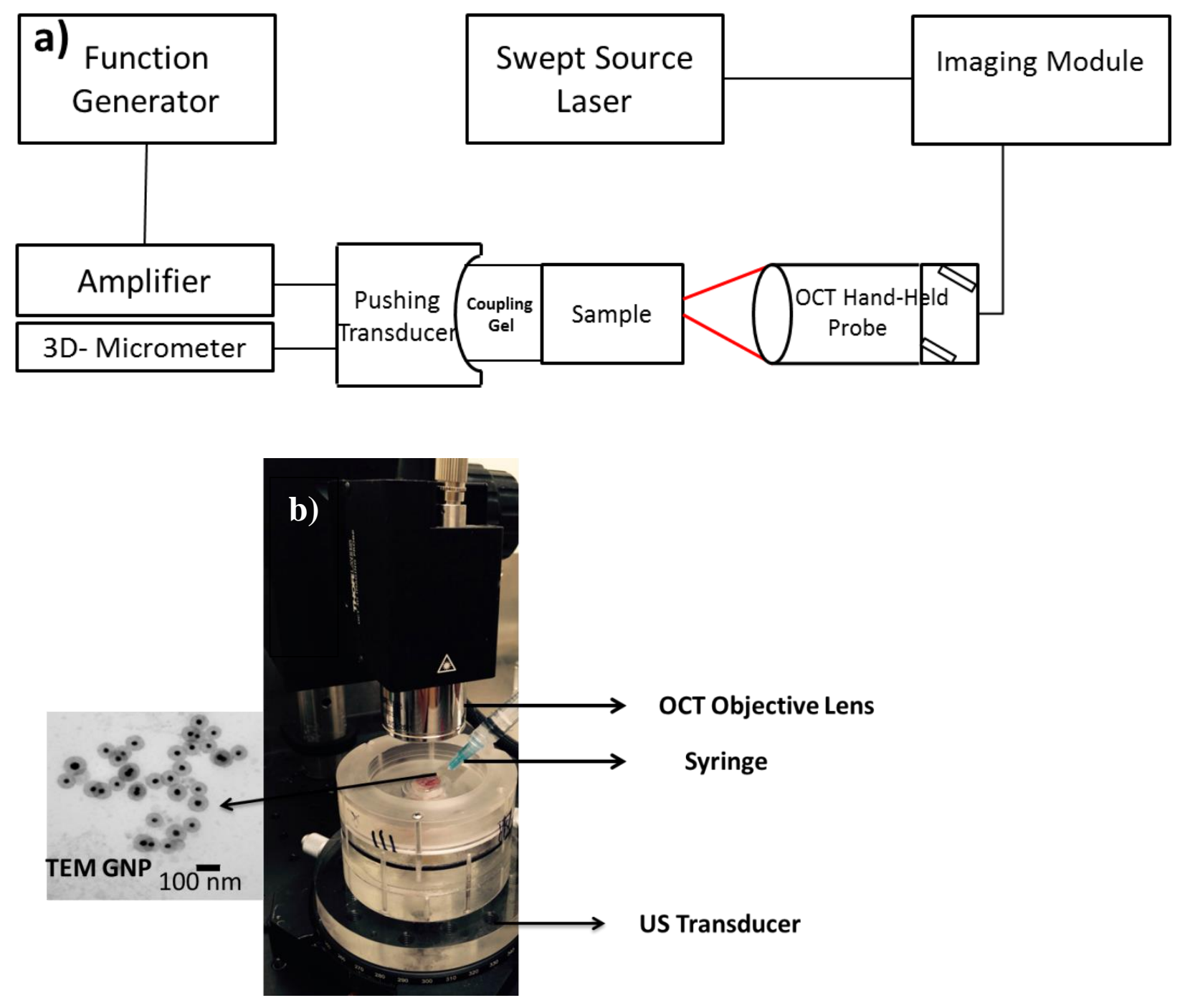

Figure 4-1 a) A schematic of the experiment set up. The ARF-OCT experimental setup consisted of the existing SS-OCT system (MEMS-VCSEL), a focused transducer (20 MHz, f-number 2.35), an amplifier and a function generator . b) Experimental setup of the ARF-OCT for the enhancement of the injected GNPS. TEM image of GNPs (scale bar is $100 \mathrm{~nm}$ ).

In order to calculate the enhanced displacement of the GNPs, 3D image volumes were taken. The enhanced transport of the GNPs can be measured by applying a simple algorithm to the speckle variance images as shown in previous work [42]. The method required the sub- 
traction of two speckle variance images, as a method to track the movement of the unresolved GNPs clusters before and after exposure to the ARF. Although an similar approach could be used based on OCT structural images, the speckle variance image has the benefit of providing higher signal from the GNPs when the GNPs are situated in highly scattering environments (e.g. tissues, cells, etc.), due to the microscopic motion of the $\mathrm{GNP}_{\mathrm{s}}$ clusters [42].

\subsection{Results}

Figure 4.2 shows the OCT speckle variance image before and after the ARF was applied to the $3 \%$ and $6 \%$ collagen and MCF7 module phantoms. The grayscale background represents the speckle variance image before the ARF was applied. The greyscale pattern in the images is representative of the complex spatial distribution of the GNPs that is the result of the GNP motion through the media and the complex interaction of the GNPs with the collagen matrix. Images were obtained when the transducer was off and then after the ARF excitation was delivered to measure the displacement of $\mathrm{GNP}_{\mathrm{s}}$ which was the result of the ARF excitation. The red pattern shows the differential of the speckle variance images before and after the ARF excitation. 

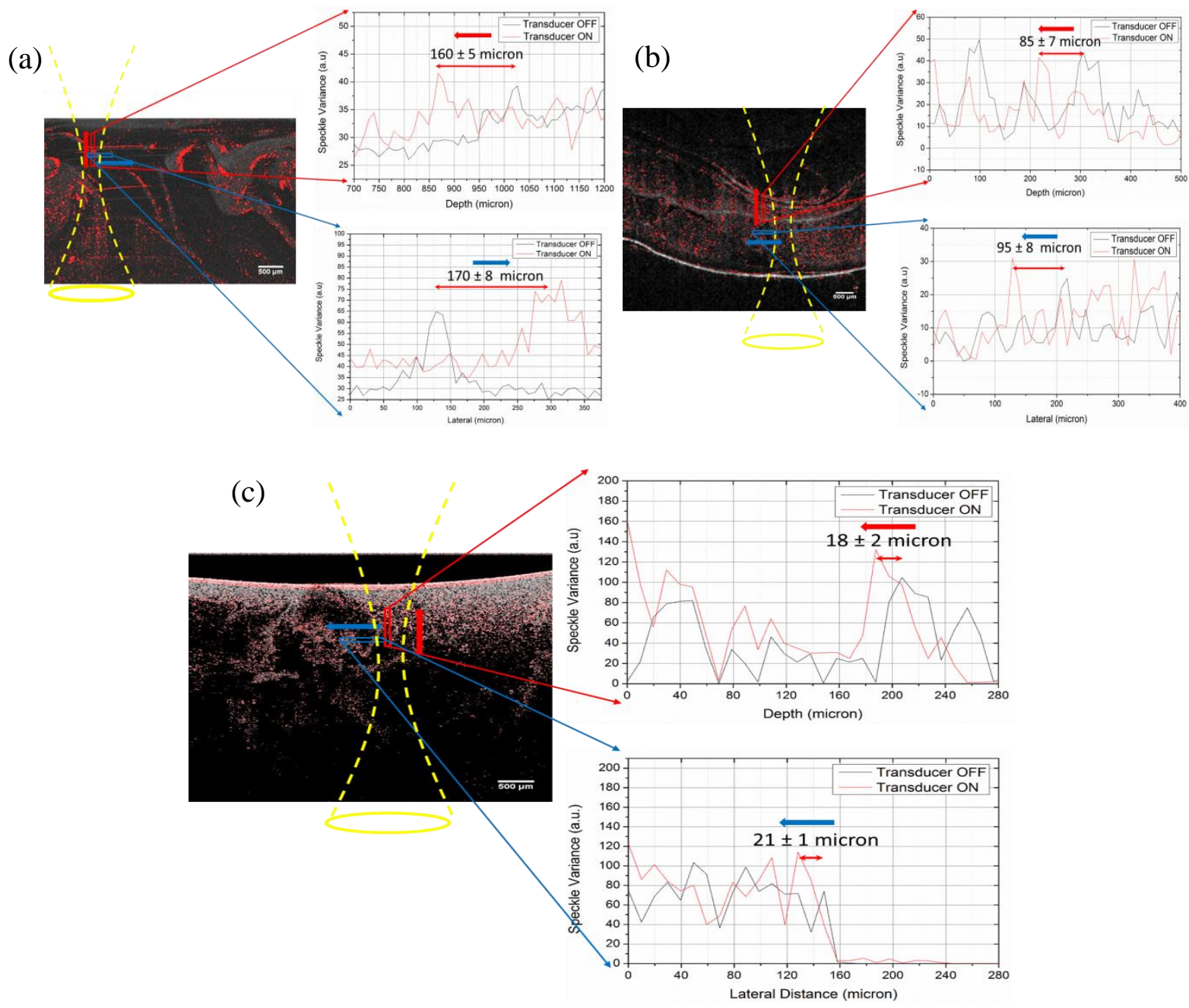

(d)

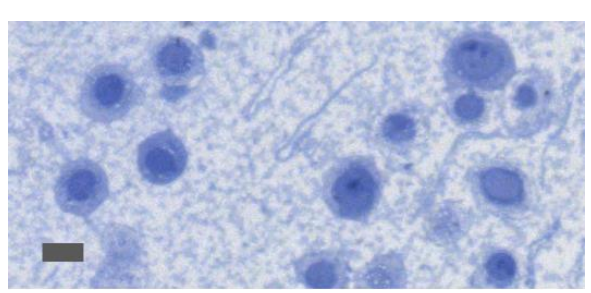

Figure 4-2 Speckle variance images of the phantoms and calculations of enhanced displacement of the GNPs in specific ROIs (red and blue rectangles). The SV images are for experiments using the (a) 3\% collagen (b) 6\% collagen (c) MCF7 Module. The red and blue arrows denote the direction of the enhanced transport. The yellow dashed lines represent the approximate location of the ultrasound beam and (d) light microscope slide of MCF7 Module and scale bar is approximately $10 \mu \mathrm{m}$. The section on the slide was 400nm thick and stained with toluidine blue. It was imaged on an iScan Coreo Au slide scanner. 
Figure 4-2 shows the measured enhanced displacement of the $\mathrm{GNP}_{\mathrm{s}}$ for the $3 \%$ and $6 \%$ collagen phantoms and the tissue engineered MCF7 constructs. These measurements were taken at locations close to the ultrasound focus. The data show the relationship between the enhanced displacement of $\mathrm{GNP}_{\mathrm{s}}$ as a function of different collagen concentrations. The ROIs are in the vicinity of the focal point of the transducer generating the ARF. The standard deviation was calculated by using three adjacent ROIs which were selected in two directions (which we term lateral and axial direction). The three ROI(s) for each direction were $50 \mathrm{mi}$ cron apart from each other. The displacement was calculated for each of the ROIs. From these three ROI(s), the average and standard deviation values in each direction were calculated. The size of each ROI is 50 micron, therefore the size of the analysis area (the three ROIs) is 100 micron. 


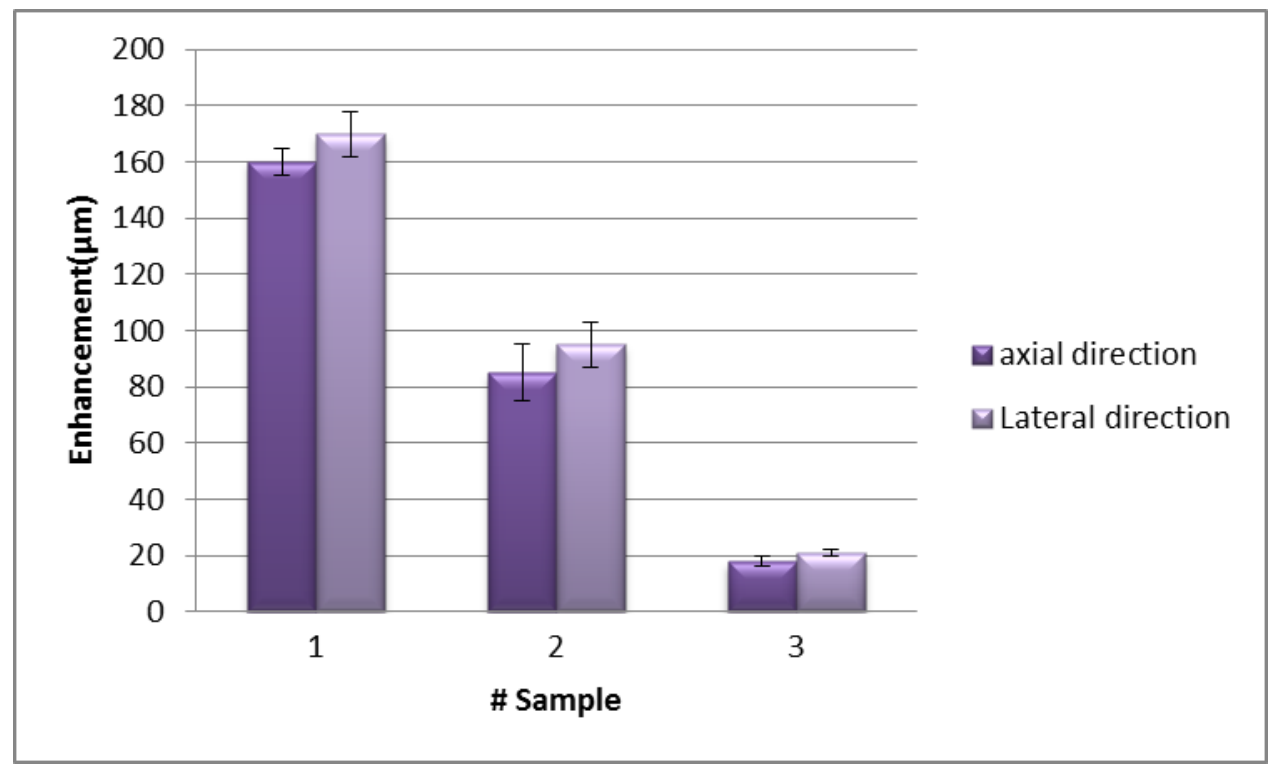

Figure 4-3 Results of the enhancement of GNP transport through phantoms of different collagen concentrations and the MCF7 module. The graph shows the relationship between enhanced displacement of $\mathrm{GNP}_{\mathrm{s}}$ in the presence of ARF as function of the collagen concentration. Samples 1, 2 and 3 represent the 3\% collagen, 6\% collagen and the MCF7 module, respectively. The ROI(s) are in the vicinity of the focal point of the transducer generating the ARF. Error bars represents the standard deviation derived from three ROIs selected in the lateral and axial directions. The three ROI(s) for each direction were 50 micron apart to each other. The displacement was calculated for each ROI. From the three ROIs, the average and standard deviation values for each direction were calculated.

We selected a region of interest (ROI) close to the ultrasound focus to calculate the displacement map (parametric map) and analyze the enhanced displacement (axial or lateral). To generate the displacement values within a ROI, we took A-lines in the lateral/axial direction and then calculated the distance between peaks of the A-line before and after the ARF to determine the displacement value associated with this one A-line. The calculated displacements for each A-line formed the parametric map. Note that in these parametric maps the lo- 
cation of only one pixel is color coded: this represents the initial location at which the displacement was calculated using the technique described above (Fig. 4.2).

The peak detection was based on identifying a large intensity gradient in intensity of the speckle variance value. The measurement was done by detecting the local maxima within the A-scan data and using the first peak from each A-scan of the speckle variance image when the ultrasound transducer is on and off, to calculate the displacements (Figure 4.2). Details of the method are provided in another publication [42]. Figure4. 4 shows a parametric map at different locations with respect to the ultrasound focus that describes the enhancement of GNP transport after the application of the ARF for 3\% collagen, $6 \%$ collagen and the MCF7 Module. 

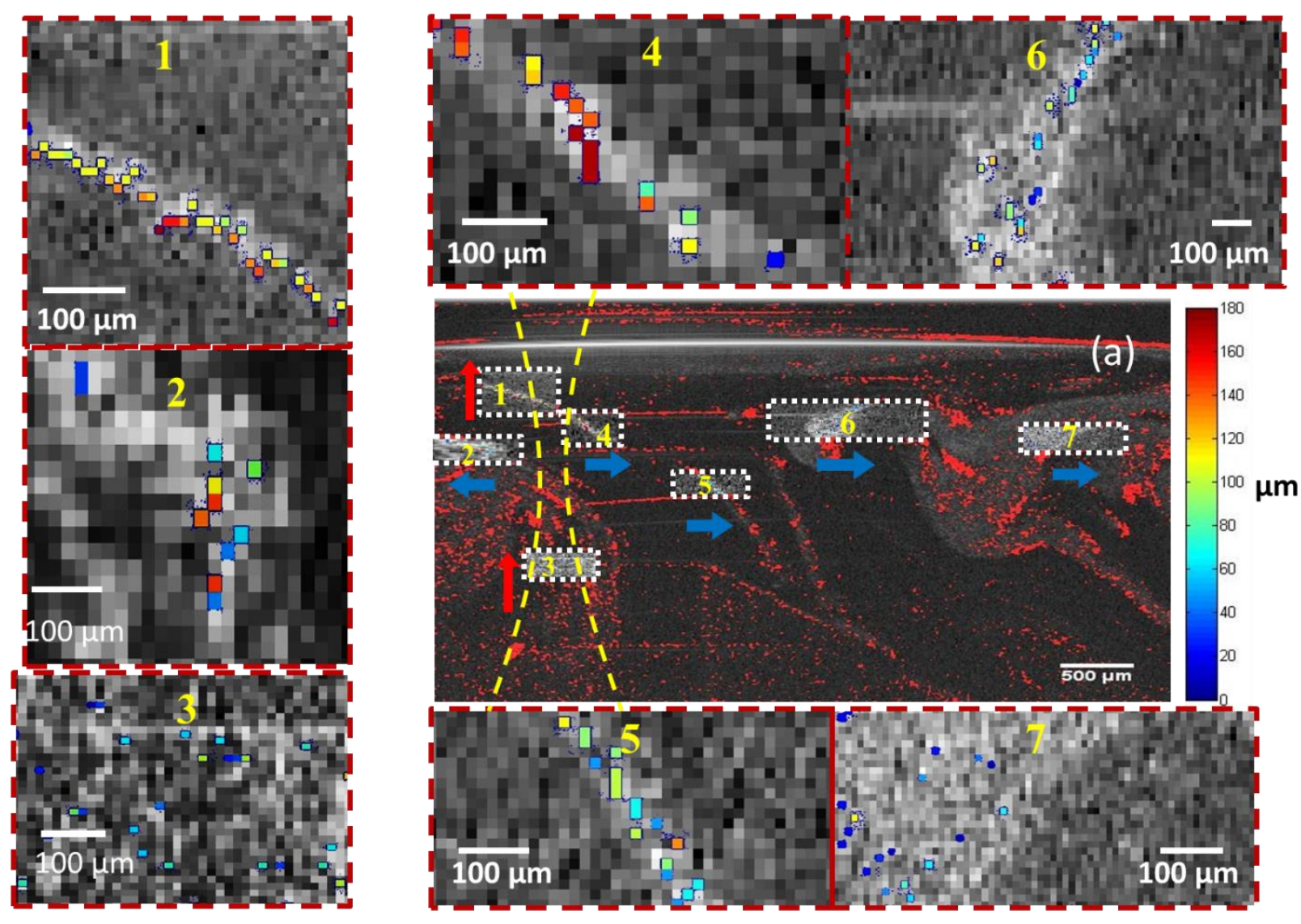


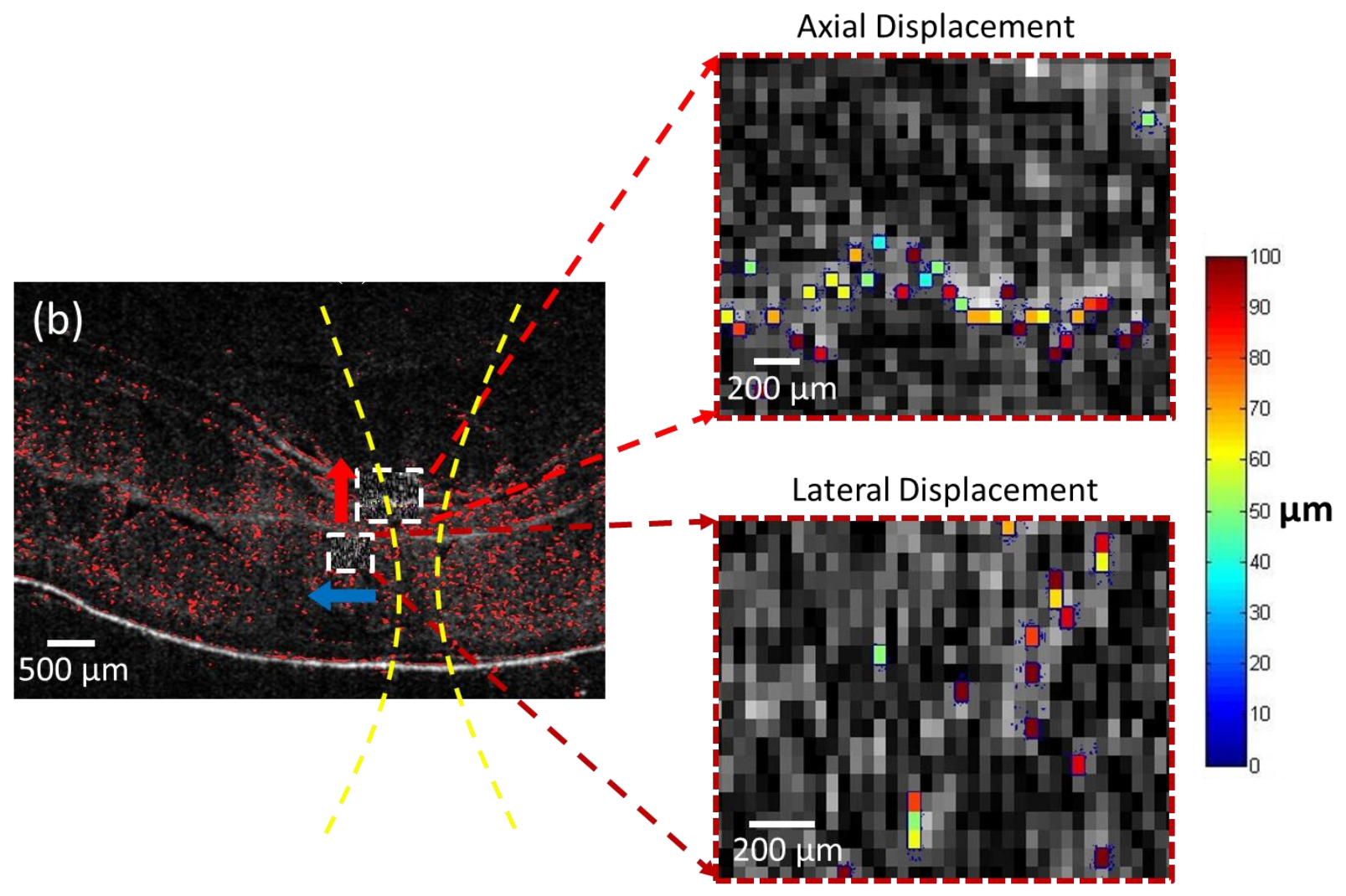




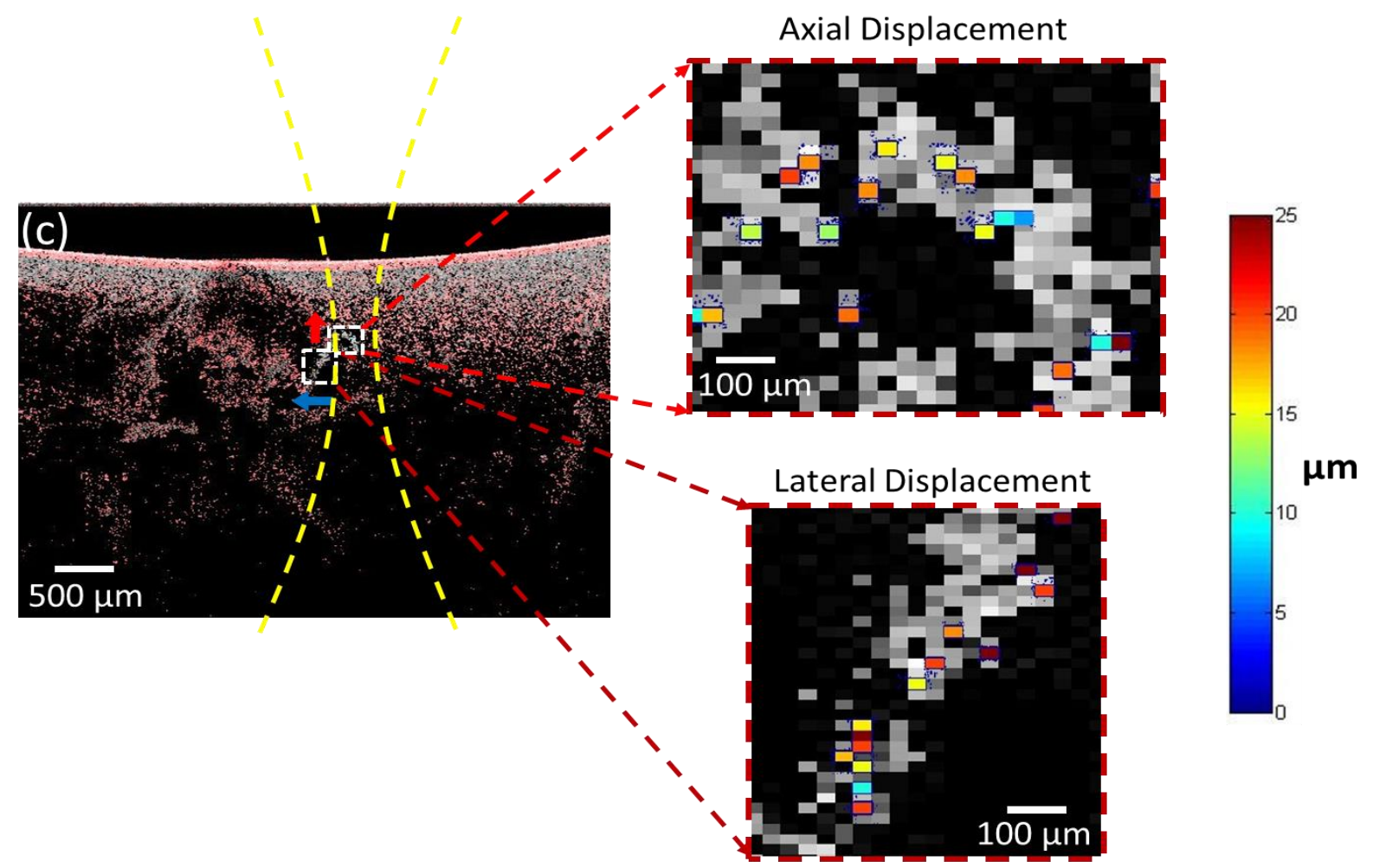

Figure 4-4 Parametric map that describes the enhanced transport of $\mathrm{GNP}_{\mathrm{s}}$ after the application of the ARF. (a) Parametric map generated in experiments with the $3 \%$ collagen phantom for different ROIs selected. ROIs \#1 and \#4 are in the vicinity of the focal point of the transducer. The average displacement within ROI \#1 is $117 \pm$ $16 \mu \mathrm{m}$ (in the axial direction) and in ROI \#4 is $161 \pm 9 \mu \mathrm{m}$ (in the lateral direction). The locations of other lateral ROIs $(\# 2,5,6)$ were chosen to demonstrate the reduction in the enhanced displacement with distance from the focal point of the transducer. Average displacement within ROI \#2 is $97 \pm 12 \mu \mathrm{m}$, \#5 is $90 \pm 9 \mu \mathrm{m}$, \#6 is 73 $\pm 8 \mu \mathrm{m}$ and \#7 is $58 \pm 12 \mu \mathrm{m}$. The average axial displacement in ROI \#3 (88 $\pm 7 \mu \mathrm{m})$ is predominantly associated with the longitudinal wave. (b) Parametric displacement map generated in experiments using the $6 \%$ collagen phantom. The ROIs were selected in the vicinity of the focal point of the transducer. Average displacement within the ROI in the axial direction is $87 \pm 9 \mu \mathrm{m}$ and the lateral direction is $90 \pm 12 \mu \mathrm{m}$. (c) Parametric displacement map generated in experiments using the MCF7 module. The ROIs were selected in the vicinity of the focal point of the transducer measuring displacements in both the axial and lateral directions and associated with the longitudinal and shear waves, respectively. The average axial displacement within the ROI is $21 \pm 11$ $\mu \mathrm{m}$ and the lateral displacement is $23 \pm 7 \mu \mathrm{m}$. The color bar represents the enhanced displacement of $\mathrm{GNP}_{\mathrm{S}}$ in $\mu \mathrm{m}$. The yellow dashed lines represents the approximate location of the ultrasound beam and where the focal point is located. 


\subsection{Discussion}

The displacements of GNPs were calculated in the vicinity of focal point in both the lateral and axial directions as shown in table 4.1. The enhanced displacement is associated with the acoustic radiation force that is generated by the change in the energy density of the acoustic field associated with both the longitudinal and the shear waves generated by the ultrasound excitation. As seen in table 4.1, the enhanced transport of the GNPs decreased as the collagen concentration increased (note that $10 \%$ collagen result are from our previous publication[42]). This is expected, as high collagen content regions impede NP motion due to steric hindrance and enhanced viscosity created by the collagen fibers. In the presence of both the collagen fibers and cells, the measured enhanced displacement went from $\sim 160 \mu \mathrm{m}$ to $\sim$ $20 \mu \mathrm{m}$ for the same ARF exposure conditions, demonstrating the difficulty in achieving delivery of GNPs when cells are surrounded by collagen networks. 
Table 4.1: Summary of the enhancement of GNPs transport through phantoms of different collagen concentrations $(3 \%, 6 \%, 10 \%[42])$ and the MCF7 module. The ROI(s) are in the vicinity of the focal point of the transducer generating the ARF. Error represents the standard deviation derived from three ROIs selected in the lateral and axial directions. The three ROI(s) for each direction were 50 micron apart to each other. The displacement was calculated for each ROI. From the three ROIs, the average and standard deviation values for each direction were calculated.

\begin{tabular}{|c|c|c|c|}
\hline Sample & Collagen & Enhanced GNR & Enhanced GNR dis- \\
& Concentration & displacement $(\mu \mathrm{m})-$ & placement $(\mu \mathrm{m})$ - lateral \\
\hline Colla- & $3 \pm 0.1$ & axial & $170 \pm 8$ \\
gen type 1 & & $160 \pm 5$ & \\
\hline Colla- & $6 \pm 0.1$ & $85 \pm 10$ & $95 \pm 8$ \\
\hline gen type 1 & & & \\
\hline Colla- & $10 \pm 0.1$ & & \\
\hline gen type 1 & & & \\
\hline MCF7 & $0.3 \pm 0.1$ & & \\
\hline & & & \\
\hline
\end{tabular}

The impulse from the US transducer creates a bulk displacement in the direction of ultrasonic beam propagation. The displacement is largest close to the transducer focus. This also creates a shear wave that propagates in a direction perpendicular to the direction of the ultrasound beam propagation. Shear waves principally propagate radially outwards from the 
focal point and perpendicular to the direction of the ARF beam. Typical values for the shear wave attenuation coefficient are two or three orders of magnitude higher than that of compressional waves [44], therefore radiation force generated by shear wave propagation (Eq.1) is expected to displace GNPs that are exposed to the shear wave.

In table 4.1, the ROI(s) in the lateral and axial directions are in the vicinity of the focal point and selected to be relatively close to each other (Figure4. 2) so that the displacements in the lateral and axial directions can be compared. It can be seen that the enhanced displacements of the GNPs in the lateral direction are comparable to those in the axial direction. There are limitations however in this comparison. Firstly, in the OCT method we have developed, the ROI must be chosen so that a sharp gradient in the intensity of the speckle variance exists. This is so that a peak can be chosen for the comparison of the speckle variance images before and after the ARF excitation. The shift in the peak location is what allows the displacement calculation. As the spatial distribution of the GNPs was not symmetric and could not be controlled, there are a limited number of regions where an appropriate ROI can be chosen for this calculation. Secondly, the way the displacements are calculated result is displacement estimates that are in only one direction (lateral or axial). Therefore, the reported displacement values are likely underestimated, as they represent displacement in one direction. We are working on algorithms that can track the displacement of the GNP cloud in the SV image in three dimensions, which would reduce this problem.

Table 4.2 highlights calculated lateral displacements based on the parametric map in Figure 4.4(a) and at different locations which are approximately at the same axial depth in 
the image. The calculated displacement decreases at locations further from the focal point. This decrease is likely due to shear wave attenuation: the reduced shear wave intensity due to attenuation results in a reduced acoustic radiation force (Eq.1) and therefore GNP displacement. We are in the process of simulating the ARF field using finite element models to compare the experimental results with these simulations [42].

Table 4.2: Summary of the lateral displacements of GNP transport after the application of the ARF in the $3 \%$ collagen phantom (Figure $4.4(\mathrm{a})$ ). $\mathrm{X}_{0}$ is in the vicinity of the focal point of the transducer which is associated with ROI \# 4 in Figure 4.4(a). The locations of ROIs: $\mathrm{X}_{0}+$ and $\mathrm{X}_{0}$ - were in the lateral direction in left side and right side of focal point and are associated to the selected lateral ROI(s) of Figure 4.4(a).

\begin{tabular}{|c|c|c|c|c|c|}
\hline Location of ROI & $\left(X_{0}-700\right) \mu \mathrm{m}$ & $\begin{array}{c}\mathrm{X}_{0} \\
(\text { vicinity of focal } \\
\text { point })\end{array}$ & $\left(\mathrm{X}_{0}+1000\right) \mu \mathrm{m}$ & $\left(\mathrm{X}_{0}+1500\right) \mu \mathrm{m}$ & $\left(\mathrm{X}_{0}+2700\right) \mu \mathrm{m}$ \\
\hline Displacement $(\mu \mathrm{m})$ & $97 \pm 12$ & $161 \pm 11$ & $90 \pm 9$ & $73 \pm 8$ & $58 \pm 12$ \\
\hline
\end{tabular}

In conclusion, we have shown that SV-OCT can be used to image the ARF induced displacements of GNPs in collagen phantoms. Displacements generated by acoustic radiation forces were measured in both axial and lateral directions, illustrating the method potential for GNPs delivery enhancement in vivo. We have demonstrated the technique in ex-vivo samples (MCF7 tissue engineered modules) and were able to measure the ARF induced GNPs displacements by using OCT. OCT is ideally suited for such measurements due to the enhanced scattering of GNPs (which provides contrast) and its excellent spatial and temporal resolutions which allow for the detection of the small enhancements in the penetration of GNPs. 


\section{Acknowledgments}

Marjan Razani is supported by Natural Sciences and Engineering Research Council of Canada (NSERC) Postgraduate Doctoral Scholarship (PGSD3).

This work is funded in part by the Natural Sciences and Engineering Research Council of Canada (NSERC discovery grant 216986-07) and Canada Foundation for Innovation (award-

ed to Dr. M. C. Kolios). We would like to thank Dr. Raffi Karshafian from the Department of Physics for his contributions and discussions.

\section{References}

1. M. D. McDonald, P. Blank, Res 62,5381-5385(2002).

2. M. O. Koo, I. Rubinstein, H. Onyuksel, Nanomedicine,1, 193-212(2005).

3. B. k.Sutradhar and A.Lutful, ISRN Nanotech, 939378(2014).

4. P. Sethi, A. Jyoti, E. P. Swindell, R. Chan, U. W. Langner, J. M. Feddock, R.Nagarajan, T. V. O'Halloran, M. Upreti, Nanotechnology, Biology and Medicine 11.8, 2013-2023(2015).

5. R. K. Jain, T. Stylianopoulos, Nature Reviews Clinical Oncology 7, 653-664 (2010).

6. R. K. Jain, Scientific American 298, 56 - 63 (2008).

7. K. Greish, Methods in Molecular Biology (Totowa, NJ, United States) 624:25-37.

8. A. J. Clark, D. T. Wiley, J. E. Zuckerman, P.Webster, J.Chao, J.Lin, Y.Yen, M. E. Davis, J. Line, Proc Natl Acad Sci U S A(2016).

9. H. Maeda, H.Nakamura, J. Fang, Advanced Drug Delivery Reviews. 65:71-9(2013).

10. F.I.Tannock, M.C.Lee, K.J.Tunggal, S.D.Cowan, J.M.Egorin,Clin Cancer Res ,8:87884(2002).

11. C. Wong, T. Stylianopoulos, J.Cui, J.Martin,V. P. Chauhan, W.Jiang, Z.Popović,R.K. Jain, M.G. Bawendi, D.Fukumura' Proc Natl Acad Sci U S A.,108:2426-2431(2011). 
12. K. P. Jain,S. K. Lee, H. I. El-Sayed , A. M. El-Sayed, J Phys Chem B,110:72387248(2006).

13. A. M. Alkilany, C. J. Murphy, J. Nanopart. Res, 12 2313-2333(2010).

14. C. Yang, M.Neshatian, M. van Prooijen, Journal of Nanoscience and Nanotechnology, 14(7), 4813-4819(2014).

15. L. Tong, Q. Wei, A. Wei, J. Cheng, Photochem Photobiol, 85(1): 21(2009).

16. H. C. Chou, C. D.Chen, C.R.C.WanG,J Phys Chem B,109:11135-11138(2005).

17. W. Jiang, Y. B. Ki, T. J. Rutka, C. W. Chan Nat Nanotechnol,3:145-50(2008).

18. D. B. Chithrani ,A. A. Ghazani, W. C.Chan, Nano Lett,6(4); 662-8(2006).

19. J. Chen, F. Saeki , J.B.Wiley , H. Cang , J.M.Cobb , Y.Z.Li , L.Au , H. Zhang , B.M.Kimmey , X.Li , Y.Xia ,Nano Lett,5(3):473-7(2005).

20. K. Huang ,H. Ma ,J. Liu ,S. Huo ,A. Kumar, T.Wei , X.Zhang ,S. Jin ,Y. Gan ,C.P. Wang ,S. He , J.X.Liang, ACS Nano,6:4483-93(2012).

21. A. L.Dykman ,G. N. Khlebtsov, ACTA Nat ,3:34-55(2011).

22. W.Cai, T.Gao,H.Hong, J.Sun' Nanotechnol Sci Appl. (1): 10.2147/NSA.S3788(2008).

23. X. Huang,S. Neretina ,A. M. El-Sayed ,Adv Mater,21:4880-910(2009).

24. S. Barua ,S. Mitragotri, Nano Today ,9:223-43(2014).

25. MI.A.inchinton,F.I. Tannock, Nature Reviews Cancer,6(8):583-92(2006).

26. J.S.Grainger,V.J. Serna ,S. Sunny ,Y. Zhou ,X.C Deng ,E.M. El-Sayed, Molecular pharmaceutics, 7(6):2006-19(2010).

27. V.Frenkel, Advanced drug delivery reviews,60(10):1193-208(2008).

28. Y.C.Lai ,Z.B. Fite ,.K. Ferrara ,Front Oncol,3:204(2013).

29. S.Wang, I. Shin, H.Hancock, B.Jang, H.Kim, S.M. Lee, V.Zderic, V.Frenkel, I.Pastan , C. Paik, M.R. Dreher,Journal of Controlled Release, 162 , 218-224(2012).

30. M. Fatemi and J. F. Greenleaf, Ultrason. Imaging, 21(2), 147-154 (1999).

31. K. Nightingale, M. S. Soo, R. Nightingale, and G. Trahey, Ultrasound Med Biol, 28(2), 227-235 (2002). 
32. G. F. Pinton and G. E. Trahey, IEEE Trans. Ultrason., Ferroelectr., Freq. Control, 53(11), 2026-2035 (2006).

33. K. R. Nightingale, R. Bentley, and G. E. Trahey, Ultrasonic Imaging, 24, 100-108 (2002).

34. L. Ostrovsky, A. Sutin, Y. Il'inskii, O. Rudenko, and A. Sarvazyan, J. Acoust. Soc. Am, 121(3), 1324-1331 (2007).

35. M. L. Palmeri, S. A. McAleavey, K. L. Fong, G. E. Trahey, and K. R. Nightingale, Ultrasonics. Ferroelectrics and Frequency Control. IEEE Transactions on, 53(11), 2065-2079 (2006).

36. A.H.Hancock, H.L.Smith, J.Cuesta, K.L.Durrani,M. Angstadt, L.M.Palmeri, E.Kimmel, V.Frenkel , Ultrasound in Medicine \& Biology,35(10):1722-36(2009).

37. E.B.O'Neill , M.Vo H, Angstadt,K. Li ,T. Quinn ,V. Frenkel ,Ultrasound in Medicine \& Biology,35(3):416-24(2009).

38. N. J. Durr, T.Larson,D. K. Smith,B. A. Korgel,K.Sokolov,A.Ben-Yakar'Nano Lett,7(4): 941-945(2007).

39. P.C. Wuytens , A.Z. Subramanian 'W.H. De Vos ' A.G. Skirtach , R.Baets, Analyst, 140, 8080-8087(2015).

40. M. Razani, A.Mariampillai, C.Sun , T.W.H. Luk, V.X.D. Yang , M.C Kolios ,Biomed. Opt. Express. 3 (5 ), 972 -980 . 2156-7085(2012).

41. A.Mariampillai,B.A. Standish .E.H.Moriyama . M.Khurana , N.R.Munce ,M.K.K. Leung ,J. Jiang,A. Cable,B.C Wilson,A.I. Vitkin,V.X .Yan ,Opt. Lett. 33(13), 15301532 (2008).

42. M.Razani, A.Zam, N.Arezza, M.C. Kolios, submitted in plos one(2016).

43. A.P.M.cGuigan, M.V.Sefton, Proc Natl Acad Sci USA,103(31),11461-11466(2006).

44. A. P. Sarvazyan, O. V. Rudenko, S. D. Swanson, J. B. Fowlkes, and S. Y. Emelianov, UMB, 24(9), 1419-1435 (1998). 


\section{Chapter 5: Discussion, Conclusions and Fu- ture Work}

\subsection{Discussion, Conclusions and Future Work}

The work in this thesis dissertation describes an acoustic radiation force shear wave technique that was combined with optical coherence tomography. This technique was used to: a) measure the mechanical properties of samples, b) enhance the transport of GNPs within tissue phantoms and monitor their spatial distribution using OCT. This chapter discusses the findings, conclusions and future work is proposed.

\subsubsection{Measurement of mechanical properties of tissue}

A SW-OCT imaging technique was proposed (Chapter 2) to enable the measurement of mechanical properties of tissue. We explored the potential of measuring shear wave propagation using optical coherence elastography (OCE) in an inhomogeneous phantom and in ex-vivo carotid artery samples based on a swept-source OCT system. The mechanical excitation produces motions within the phantom that can be used for the estimation of mechanical properties using SW-OCE. I presented a technique for calculating tissue mechanical properties by propagating shear waves in inhomogeneous tissue equivalent phantoms and carotid artery samples using the ARF of an ultrasound transducer, and measuring the shear wave speed and associated properties in the different layers with OCT phase maps. The displace- 
ments of shear waves at each tracking location are calculated with a speckle-tracking algorithm based on the OCT phase maps generated. The Young's moduli and shear moduli were calculated using OCT phase maps.

\subsubsection{Phase noise of SS-OCT systems}

Typically SS-OCT systems have higher phase noise than SD-OCT systems, especially at high A-scan rates $[84,85]$. However, the phase noise of the relatively low speed SSOCT ( $8 \mathrm{kHz}$ bi-directional) used in the experiments was sufficient to measure phase changes induced by shear wave propagation. The OCT system was very sensitive to small vibrations and electronics device noise, which had a negative impact on the phase stability. To minimize this effect, a vibration isolation optical table was set up.

\subsubsection{Conclusions}

We have demonstrated, for the first time, an SW-OCE technique that uses ARF for mechanical excitation to measure shear wave propagation in an inhomogeneous phantom and in carotid artery samples. This novel method illustrates the potential of the technique for the mechanical characterization of tissues in vivo. This method lays the foundation for future invitro and in-vivo studies of mechanical property measurements of biological tissues such as vascular tissues, where normal and pathological structures may exhibit significant contrast in the shear modulus at small spatial scales.

The measurement of the elastic properties of the adventitia demonstrates how the superior spatial resolution of OCT can be used in the SW-OCE approach to interrogate tissue 
structures that are too small to be resolved with other competing imaging methods. Tissue elasticity may be thought of simply as the relative hardness or softness of a biomaterial, and clinical examination by palpation is a traditional and highly effective means of detecting pathologies such as coronary artery disease, which demonstrates local elastic inhomogeneity. OCT provides greater spatial and phase resolution than previous methods that have been used for the study of the deformation of tissue and biomaterials.

\subsubsection{Future Work: Measurement of mechanical properties in-vivo}

The OCE method proposed in Chapter 2 can be applied in vivo for applications such as breast cancer margin detection or the characterization of plaques by mapping the mechanical properties of tissue. In order to implement this method in vivo, the technique would require implementation using a catheter/ probe system to reach the target site, due to the limited penetration depth of OCT [86]. Other investigators have developed such systems [8789]. This newly developed technique has many potential applications, including identifying the mechanical properties of other tissues (e.g. such as ocular tissue) and identifying changes in the mechanical properties associated with arterial disease and cancer during treatment as a method of treatment monitoring.

\subsubsection{Enhanced GNP displacement}

In the second part of this thesis (chapter 3 and 4), I presented a technique to image the enhanced GNP displacement generated using an acoustic radiation force (ARF), this time as an excitation source to transport GNPs though collagen based phantoms. Differential OCT 
speckle variance images with and without the ARF excitation were used to estimate the nanoparticle displacement generated by the ARF. Using this OCT imaging technique, the ARF assisted enhanced transport of particles though a porous membrane and a collagen gel were analysed. I was able to visualize and characterize the acoustic radiation force assisted enhanced displacement of the GNPs. The enhanced displacement of GNPs was measured in phantoms with different collagen concentrations and a tissue engineered construct containing MCF7 cells (Chapters 3 and 4). Acoustic radiation force generated shear waves were propagated through collagen phantoms of different concentrations (3\%,6\% and 10\%) and tissue engineered constructs after $\mathrm{GNP}_{\mathrm{S}}$ were injected into them. By using SW-OCT, the enhanced displacement of the GNPs was measured.

\subsubsection{Lateral displacements}

Shear waves can be generated by using a focused impulse generated by an ultrasound transducer. Due to the high attenuation of shear waves, it is possible to generate the shear wave within a very limited area of tissue, typically localized to near the focal point of the ultrasonic beam [9]. Shear waves were used for the enhancement of the transport of GNPs through collagen gels or tissue engineered MCF7 modules. In Chapter 4, the results showed that the enhanced GNP displacement in the lateral direction at different locations decayed as ROIs were selected further away from the transducer focus. The reduced GNP displacement further from the focus shows the effect of attenuation of the shear wave in the lateral direction. Table 5.1 highlights calculated lateral displacements at different locations, which are approximately at the same axial depth in the image. The calculated displacement decreases 
at locations further from the focal point. This decrease is likely due to shear wave attenuation: the reduced shear wave intensity due to attenuation results in a reduced acoustic radiation force $\left(F=\frac{2 \alpha I}{C}\right)$ and therefore GNP displacement. Such displacements using shear waves to enhance GNP transport have not been reported before.

Table 5.1: Summary of the lateral displacements of GNP transport after the application of the ARF in the $3 \%$ collagen phantom, $\mathrm{X}_{0}$ is in the vicinity of the focal point of the transducer.

\begin{tabular}{|c|c|c|c|c|c|}
\hline Location of ROI & $\left(\mathrm{x}_{0}-700\right) \mu \mathrm{m}$ & $\begin{array}{c}\mathrm{x}_{0} \\
\text { (vicinity of focal } \\
\text { point) }\end{array}$ & $\left(\mathrm{x}_{0}+1000\right) \mu \mathrm{m}$ & $\left(\mathrm{x}_{0}+1500\right) \mu \mathrm{m}$ & $\left(\mathrm{x}_{0}+2700\right) \mu \mathrm{m}$ \\
\hline Displacement $(\mu \mathrm{m})$ & $97 \pm 12$ & $161 \pm 11$ & $90 \pm 9$ & $73 \pm 8$ & $58 \pm 12$ \\
\hline
\end{tabular}

\subsubsection{Motion detection}

Two general approaches can be used to detect motion using the backscattered light in OCT. One of the techniques is based on speckle variance. The other technique is based on phase maps generated by processing the OCT signals. Even though in both techniques the backscattered OCT signals are used to detect the motion, the speckle variance OCT enables visualization of slow flow and is independent of any Doppler angle. Therefore this approach should be the most appropriate method to measure the slow movements of transported GNPs. Moreover, if the OCT system used does not have a sufficient phase stability, the SV is a more robust method as it is generally does not depend on the phase stability of the imaging system. In this thesis, I have used the phase technique to measure the very small motion of 
the medium (tissue) in chapter 2. This motion is associated with the motion of the medium perpendicular to the direction of the travelling shear wave, and is in the nanometre range. For the application related to the enhanced transport of the GNPs, where motions are at greater scales and phase stability is not as an important issue, I used the speckle variance to measure the motion. This work is described in Chapter 3 and 4. Speckle variance OCT imaging has been shown to be sensitive to the microscopic motion of sub-resolution scatterers.

\subsubsection{Concentration Effect}

The effects of different collagen concentrations on the ARF induced displacement of GNPs was examined in this thesis. Results showed that GNP transport was less effective with increasing concentrations of collagen. The displacements were calculated in two different directions (lateral and axial) near the ultrasound transducer focus. Table 5.2 summarizes the enhancement of GNPs transport through phantoms of different collagen concentrations (3\%, $6 \%, 10 \%)$ and the MCF7 module. 
Table 5.2: Summary of the enhancement of GNPs transport through phantoms of different collagen concentrations $(3 \%, 6 \%$ and $10 \%)$ and the MCF7 module.

\begin{tabular}{lccc}
\hline Sample & $\begin{array}{c}\text { Collagen } \\
\text { Concentration }(\%)\end{array}$ & $\begin{array}{c}\text { Enhanced GNR dis- } \\
\text { placement }(\mu \mathrm{m}) \text { - axial }\end{array}$ & $\begin{array}{c}\text { Enhanced GNR displace- } \\
\text { ment }(\mu \mathrm{m}) \text { - lateral }\end{array}$ \\
\hline Collagen type 1 & $3 \pm 0.1$ & $160 \pm 5$ & $170 \pm 8$ \\
Collagen type 1 & $6 \pm 0.1$ & $85 \pm 10$ & $95 \pm 8$ \\
Collagen type 1 & $10 \pm 0.1$ & $62 \pm 6.5$ & $65 \pm 5$ \\
MCF7 Module & $0.3 \pm 0.1$ & $18 \pm 2$ & $21 \pm 1$ \\
\hline
\end{tabular}

These measurements can be thought to roughly translate to different tissue types due to fact that different tissues (or diseased tissue states) have different collagen concentrations. The amount of ARF assisted GNP transport for a given tissue type is strongly dependent on collagen concentration. This study showed that by using this technique it may be possible to enhance GNP penetration in different tissues.

In table 5.2, the ROI(s) in the lateral and axial directions are near the focal point and selected to be relatively close to each other so that the displacements in the lateral and axial directions can be compared. The enhanced displacements of the GNPs in the lateral direction are comparable to those in the axial direction. 


\subsubsection{Measurement methods}

In the first part of this study, SS-OCT with an A-scan rate of $8 \mathrm{KHz}$ and lateral resolution $13 \mu \mathrm{m}$ was used. It should be noted that the maximum displacement was calculated approximately $0.1 \mu \mathrm{m}$. This displacement was calculated based on the phase of the signal. Phase sensitive methods have been used by others [90] to obtain sub-wavelength displacement sensitivity for imaging biological samples. Another group [91] used a method similar to that presented in this thesis to show that phase-sensitive optical coherence tomography (PhS-OCT) can detect and track, temporally and spatially, shear wave propagation within tissue that was induced by ultrasound radiation force. The use of phase sensitive techniques allow displacement measurements that could be below the imaging resolution of the system and that is given by the displacement equation $\left(Z=\frac{\lambda_{0} \Delta \varphi}{4 \pi n}\right)$ where $\lambda_{0}$ is the center wavelength and $n$ is the sample refractive index.

In the second part of this study, a SS-OCT system with an A-scan rate of $100 \mathrm{KHz}$ and a lateral resolution $25 \mu \mathrm{m}$ was used. The displacement measurements were not based on phase sensitive techniques. We used Speckle Variance methods which are based on speckle intensity changes as a function of time to measure the enhanced displacement of transported nanoparticles. The error bar represents the measurements error which is standard deviation of 3 measurements in closely placed ROIs. The standard deviation was calculated by using three adjacent ROIs which were selected in two directions (which we term lateral and axial directions). The three ROI(s) for each direction were 50 micron apart from each other. The dis- 
placement was calculated for each of the ROIs. From these three ROI(s), the average and standard deviation values in each direction were calculated.

\subsubsection{Conclusions}

I demonstrated a shear wave ultrasound and optical coherence tomography method to measure the spatial distribution of GNPs through collagen-based phantoms and to monitor the enhanced transport of the GNPs though these phantoms. Speckle variance OCT is a measurement technique that relies on the decorrelation of the OCT signal and enables visualization of slow moving scatterers, independent of Doppler angle. We have used this approach to examine the microscopic movement of GNP and their net displacement after ultrasound excitation. The high spatial resolution of OCT allows for the detection of the small enhancements in NP penetration in the samples that are important to the assessment of the enhanced NP delivery.

\subsubsection{Future work: Acoustic radiation force enhanced displacement of nanoparticles}

The SW-OCT method developed should next be translated into animal models to examine if the results in the tissue equivalent phantoms can be realized in-vivo.

Moreover, nano particles with varied size and shape could be used to investigate the spatial distributions of nanoparticles in ex vivo and in vivo models and investigate how ARF assisted enhanced delivery works for the different combinations of nanoparticles and model environments. Theoretical models should be developed to accurately model this phenomenon so as to optimize the US exposure parameters (e.g. the beam width of the excitation which 
dictates the frequency content of the shear wave produced). Strong focusing also limits the excitation region to smaller tissue regions, which is an important consideration for in-vivo studies. In future work a collection of transducers with different f-numbers should be used to produce different excitation patterns and shear waves of different properties (e.g. frequencies) to examine how this would affect the transport of the GNPs. The design of such transducers will depend on the application envisioned and the scales of the heterogeneities that need to be imaged (such as in breast cancer) and for which the enhanced delivery is required. Further testing in-vivo is required to fully demonstrate the method of shear wave speckle variance (SW-SV) as a method to measure the enhancement of transported NPs in realistic tissue environments and in the presence of flowing blood. We present the OCT measurements of the nanoparticle enhanced displacements in the collagen based samples as a stepping stone for expanding the research into observing enchantment of delivery into tumours, in-vivo. 


\section{Bibliography}

[1] C. Amador, M. W. Urban, S. Chen, Q. Chen, K-N. An, and J. F. Greenleaf, "Shear elastic modulus estimation from indentation and SDUV on gelatin phantoms" IEEE Trans. Biomed.Eng, 58(6), 1706 - 1714 (2011).

[2] Kevin J. Glaser, Joel P. Felmlee,2Armando Manduca, Yogesh Kannan Mariappan,and Richard L. Ehman,Stiffness-Weighted Magnetic Resonance Imaging, Magnetic Resonance in Medicine 55:59-67 (2006).

[3] J. Ophir, S. K. Alam, B. Garra, F. Kallel, E. Konofagou, T. Krouskop, and T. Varghese, "Elastography: ultrasonic estimation and imaging of the elastic properties of tissues," Proc Inst Mech Eng H, 213(3), 203- 233 (1999).

[4] Jonathan Bishop, Greg Poole Michael Leitch, BSc Donald B. Plewes," Magnetic resonance imaging of shear wave propagation in excised tissue" Magnetic Resonance Imaging, 8(6), 1257-1265 (1998).

[5] M. L. Palmeri, M. H. Wang, J. J. Dahl, K. D. Frinkley, and K. R. Nightingale, "Quantifying hepatic shear modulus in vivo using acoustic radiation force," Ultrasound Med. Biol, 34(4), 546-558 (2008).

[6] S. L. Floc'h, G. Cloutier, G. Finet, P. Tracqui, R. I. Pettigrew, and J. Ohayon, "On the potential of a new IVUS elasticity modulus imaging approach for detecting vulnerable atherosclerotic coronary plaques: in vitro vessel phantom study," Phys. Med. Biol. 55, 5701-5721 (2010). 
[7] M. Razani, A. Mariampillai, C. Sun, T. W. H. Luk, V. X. D. Yang, and M. C. Kolios, "Feasibility of optical coherence elastography measurements of shear wave propagation in homogeneous tissue equivalent phantoms," Biomed. Opt. Express 3(5), 972980 (2012).

[8] C. Sun, B. Standish, and V. X. D. Yang, "Optical coherence elastography: current status and future applications," J. Biomed. Opt. 16(4), 043001 (2011).

[9] J. Li, S. Wang, K. R. Manapuram, M. Singh, F. M. Menodiado, S. Aglyamov,S. Emelianov, M. D. Twa, and K. V. Larina,” Dynamic optical coherence tomography measurements of elastic wave propagation in tissue-mimicking phantoms and mouse cornea in vivo,” J. Biomed. Opt. 18 (12), 121503 (2013).

[10] Dayton, Paul, Alexander Klibanov, Gary Brandenburger, and Kathy Ferrara. "Acoustic radiation force in vivo: a mechanism to assist targeting of microbubbles." Ultrasound in medicine \& biology 25(8): 1195-1201(1999).

[11] Hilary A. Hancock, Lauren H. Smith, Julian Cuesta, Amir K. Durrani, Mary Angstadt, Mark L. Palmeri, Eitan Kimmel, and Victor Frenkel "Investigations into pulsed-high intensity focused ultrasound enhanced delivery: Preliminary evidence for a novel mechanism" Ultrasound Med Biol. 35(10): 1722-1736(2009).

[12] Paul A. Dayton, Shukui Zhao,Susannah H. Bloch, Pat Schumann,Kim Penrose, Terry O. Matsunaga, Reena Zutshi, Alexander Doinikov, and Katherine W. Ferrara “Application of Ultrasound to Selectively Localize Nanodroplets for Targeted Imaging and Therapy", Mol Imaging. 5(3): 160-174(2006). 
[13] Böhmer, M. R., et al. "Focused ultrasound and microbubbles for enhanced extravasation." Journal of Controlled Release 148.1 18-24(2010).

[14] Klibanov, Alexander L. "Microbubble contrast agents: targeted ultrasound imaging and ultrasound-assisted drug-delivery applications." Investigative radiology 41.3: 354-362(2006).

[15] Al-Bataineh, Osama, Jürgen Jenne, and Peter Huber. "Clinical and future applications of high intensity focused ultrasound in cancer." Cancer treatment reviews 38.5: 346$353(2012)$.

[16] Nicholas J. Durr, Timothy Larson, Danielle K. Smith, Brian A. Korgel, Konstantin Sokolov,and Adela Ben-Yakar "Two-Photon Luminescence Imaging of Cancer Cells using Molecularly Targeted Gold Nanorods" Nano Lett. Apr; 7(4): 941-945(2007).

[17] Pieter C. Wuytens, Ananth Z. Subramanian 'Winnok H. De Vos 'Andre G. Skirtach and Roel Baets, "Gold nanodome-patterned microchips for intracellular surfaceenhanced Raman spectroscopy”, Analyst, , 140, 8080-8087(2015).

[18] Blackmon, Richard L., Rupninder Sandhu, Brian S. Chapman, Patricia CasbasHernandez, Joseph B. Tracy, Melissa A. Troester, and Amy L. Oldenburg. "Imaging Extracellular Matrix Remodeling In Vitro by Diffusion-Sensitive Optical Coherence Tomography." Biophysical Journal 110(8): 1858-1868(2016).

[19] Tatsuya Nakamura, Fuminori Sugihara, Hisashi Matsushita, Yoshichika Yoshioka, Shin Mizukami and Kazuya Kikuchi, "Mesoporous silica nanoparticles 
for magnetic resonance imaging, fluorescence imaging, and drug delivery”, Chem. Sci ,6, 1986-1990(2015).

[20] Mariampillai A., "Development of a high-resolution microvascular imaging toolkit for optical coherence tomography," PhD Thesis, University of Toronto (2010).

[21] Murtaza Ali and Renuka Parlapalli, "Signal Processing Overview of Optical Coherence Tomography Systems for Medical Imaging” Texas Instruments,June (2010).

[22] Darren Morofke, “ 2-D kasai velocity estimation for Doppler optical coherence tomography", Thesis,Ryerson University (2006).

[23] C. W. Sun, S. Y. Lee and K. F. Lin, "Review: Optical scanning probe for optical coherence tomography,” J. Med. Biol. Eng., 34: 95-100 (2014).

[24] Duma, V.-F., Dobre, G., Demian, D., Cernat, R., Sinescu, C., Topala, F. I., Negrutiu, M. L., Hutiu Gh., Bradu, A., and Podoleanu, A. Gh., "Handheld scanning probes for optical coherence tomography," Romanian Reports in Physics 67(4) (2015).

[25] Duma, V.-F., Demian, D., Sinescu, C., Negrutiu, M. L., Cernat, R., Topala, F. I., Hutiu, Gh., Bradu, A., and Podoleanu, A. Gh., "Handheld scanning probes for OCT: Developments, applications and perspectives," Proc. SPIE (2015).

[26] Mariampillai, B. A. Standish, E. H. Moriyama, M. Khurana, N. R. Munce, M. K. K. Leung, J. Jiang, A. Cable, B. C. Wilson, I. A. Vitkin, and V. X. D. Yang, "Speckle variance detection of microvasculature using swept-source optical coherence tomography," Opt. Lett. 33(13), 1530-1532 (2008). 
[27] S. G. Adie, X. Liang, B. F. Kennedy, R. John, D. D. Sampson, and S. A. Boppart, “ Spectroscopic optical coherence elastography,” Opt. Express,18(25),2551925534(2010).

[28] Bercoff J, Criton A, Bacrie CC, et al. "ShearWaveTM Elastography: a new real time imaging mode for assessing quantitatively soft tissue viscoelasticity”,. Proceedings of the 2008 IEEE International Ultrasonics Symposium, Beijing, China, November 2$5,(2008)$.

[29] Fink M, Tanter M: "Multiwave imaging and super resolution". Phys Today 2010, 63:28-33(2010).

[30] J. Bercoff, M. Tanter, and M. Fink, "Supersonic shear imaging: A new technique for soft tissue elasticity mapping," IEEE Trans. Ultrason., Ferroelectr., Freq. Control, 51(4), 396 - 409 (2004).

[31] A. Karpiouk, S. Aglyamov, Y. Ilinskii, E. Zabolotskaya, and S. Emelianov, “Assessment of shear modulus of tissue using ultrasound radiation force acting on a spherical acoustic inhomogeneity,” IEEE Trans. Ultrason., Ferroelectr., Freq. Control, 56(11), 2380 - 2387 (2009).

[32] http://www.mayoclinic.org/magnetic-resonance-elastography).(Access April 2016)

[33] Tanter, Mickael, Jeremy Bercoff, Alexandra Athanasiou, Thomas Deffieux, Jean-Luc Gennisson, Gabriel Montaldo, Marie Muller, Anne Tardivon, and Mathias Fink. "Quantitative assessment of breast lesion viscoelasticity: initial clinical results using 
supersonic shear imaging." Ultrasound in medicine \& biology 34, no. 9: 13731386(2008).

[34] K. Nightingale, S. McAleavey, and G. Trahey, "Shear wave generation using acoustic radiation force: In vivo and ex vivo results,"Ultrasound Med. Biol, 29(2), 1715-1723 (2003).

[35] M. L. Palmeri, S. A. McAleavey, G. E. Trahey, and K. R. Nightingale, "Ultrasonic tracking of acoustic radiation force-induced displacements in homogeneous media," IEEE Trans. Ultrason., Ferroelectr., Freq. Control, 53(7), 1300-1313 (2006).

[36] G. F. Pinton and G. E. Trahey, "Continuous delay estimation with polynomial splines,” IEEE Trans. Ultrason., Ferroelectr., Freq. Control, 53(11), 2026-2035 (2006).

[37] J. McLaughlin and D. Renzi, "Shear wave speed recovery in transient elastography and supersonic imaging using propagating fronts,"Inverse Probl, 22, 681-706 (2006).

[38] J. McLaughlin and D. Renzi, "Using level set based inversion of arrival times to recover shear wave speed in transient elastography and supersonic imaging," Inverse Probl, 22, 707-725 (2006).

[39] P. Sarvazyan, O. V. Rudenko, S. D. Swanson, J. B. Fowlkes, and S. Y. Emelianov, "Shear wave elasticity imaging: A new ultrasonic technology of medical diagnostics," UMB, 24(9), 1419-1435 (1998). 
[40] Arda, Kemal, Nazan Ciledag, Elif Aktas, Bilgin Kadri Arıbas, and Kenan Köse. "Quantitative assessment of normal soft-tissue elasticity using shear-wave ultrasound elastography." American Journal of Roentgenology, 197(3): 532-536(2011).

[41] Lupsor, Monica, et al. "Performance of a new elastographic method (ARFI technology) compared to unidimensional transient elastography in the noninvasive assessment of chronic hepatitis C. Preliminary results." J Gastrointestin Liver Dis 18.3, 303-310. (2009).

[42] Huang, Chih-Chung, Cho-Chiang Shih, Ting-Yu Liu, and Po-Yang Lee. "Assessing the viscoelastic properties of thrombus using a solid-sphere-based instantaneous force approach." Ultrasound in medicine \& biology 37(10) :1722-1733(2011).

[43] Doherty, Joshua R., Gregg E. Trahey, Kathryn R. Nightingale, and Mark L. Palmeri. "Acoustic radiation force elasticity imaging in diagnostic ultrasound."Ultrasonics, Ferroelectrics, and Frequency Control, IEEE Transactions 60(4),: 685-701(2013).

[44] Tanter, Mickaël, David Touboul, Jean-Luc Gennisson, Jeremy Bercoff, and Mathias Fink. "High-resolution quantitative imaging of cornea elasticity using supersonic shear imaging." Medical Imaging, IEEE Transactions 28(12): 1881-1893(2009).

[45] Glaser KJ, Felmlee JP, Ehman RL. "Rapid MR elastography using selective excitations.” Magn Reson Med. 55:1381-1389(2006).

[46] Shadi F. Othman, Xiaohong Joe Zhou, Huihui Xu, Thomas J. Royston, Richard L. Magin. "Error propagation model for microscopic magnetic resonance elastography shear-wave images” Magnetic Resonance Imaging 25 94-100(2007). 
[47] Nguyen, Thu-Mai, et al. "Shear wave elastography using amplitude-modulated acoustic radiation force and phase-sensitive optical coherence tomography." Journal of biomedical optics 20.1: 016001-016001(2015).

[48] Han, Zhaolong, et al. "Quantitative methods for reconstructing tissue biomechanical properties in optical coherence elastography: a comparison study." Physics in medicine and biology $60.9: 3531(2015)$.

[49] Wang, Shang, and Kirill V. Larin. "Optical coherence elastography for tissue characterization: a review." Journal of biophotonics $8.4: 279-302(2015)$.

[50] Mulligan, Jeffrey A., et al. "Emerging Approaches for High-Resolution Imaging of Tissue Biomechanics With Optical Coherence Elastography."Selected Topics in Quantum Electronics, IEEE Journal of 22.3: 1-20(2016).

[51] X. Liang, M. Orescanin, K. S. Toohey, M. F. Insana, and S. A. Boppart, "Acoustomotive optical coherence elastography for measuring material mechanical properties," Opt. Letters, 34(19), 2894-2896 (2009).

[52] X. Liang, S. G. Adie, R.John, and S. A. Boppart, "Dynamic spectral-domain optical coherence elastography for tissue characterization," Opt. Express,18(13),1418314190(2010).

[53] B. F. Kennedy, X. Liang, S. G. Adie, D. K. Gerstmann, B. C. Quirk, S. A. Boppart, and D.D. Sampson1, " In vivo three-dimensional optical coherence elastography," Opt. Express,19(7),6623-6634(2011). 
[54] X. Liang and S.A. Boppart, "Biomechanical properties of in vivo human skin from dynamic optical coherence elastography”,57(4), 953-959(2010).

[55] B.F. Kennedy, T. R. Hillman, R. A. McLaughlin, B. C. Quirk, and D. D. Sampson, "In vivo dynamic optical coherence elastography using a ring actuator," Opt. Express,,17(24),21762-21772(2009).

[56] Coutts L. V., Miller N. R., Harland C. C., Bamber J. C., "Feasibility of skin surface elastography by tracking skin surface topography,” J. Biomed. Opt. 18(12), 121513 (2013).

[57] S. H. Yun, G. J. Tearney, J. F. de Boer, and B. E. Bouma, "Motion artifacts in optical coherence tomography with frequency-domain ranging," Opt. Express,12(13),29772998(2004).

[58] Mariampillai, B. A. Standish, N. R. Munce, C. Randall, G. Liu, J. Y. Jiang, A. E. Cable, I. A. Vitkin, and V. Yang, "Doppler optical cardiogram gated 2D color flow imaging at $1000 \mathrm{fps}$ and $4 \mathrm{D}$ in vivo visualization of embryonic heart at $45 \mathrm{fps}$ on a swept source," Opt. Express, 15( 4), 1627-1638 (2007).

[59] Bercoff, J., M. Pernot, M. Tanter, and M. Fink. "Monitoring thermally-induced lesions with supersonic shear imaging." Ultrasonic imaging 26(2): 71-84(2004).

[60] McDonald DM, Blank P. "Significance of blood vessel leakiness in cancer.Cancer" Res ;62:5381-5385(2002).

[61] Triantafyllos Stylianopoulos, Rakesh K. Jain “ Design considerations for nanotherapeutics in oncology", Nanomedicine,11:1893-1907 (2015). 
[62] A.B. Etame, Enhanced Delivery of Gold Nanoparticles with Therapeutic Potential for Targeting Human Brain Tumors (Ph.D. thesis), University of Toronto (2012).

[63] Sarin H. "Physiologic upper limits of pore size of different blood capillary types and another perspective on the dual pore theory of microvascular permeability”,J.Angiogenes Res.;2:14. (2010).

[64] N Rapoport "Phase-shift, stimuli-responsive perfluorocarbon nanodroplets for drug delivery to cancer". Wiley Interdiscip Rev Nanomed Nanobiotechnol;4:492510(2012).

[65] Yohan, D. and B.D. Chithrani, Applications of nanoparticles in nanomedicine. Journal of Biomedical Nanotechnology, 10(9): p. 2371-2392 (2014).

[66] Jiang W, Kim BY, Rutka JT, Chan WC. "Nanoparticle-mediated cellular response is sizedependent”. Nat Nanotechnol;3:145-50 (2008).

[67] Chithrani, B.D., A.A. Ghazani, and W.C. Chan, "Determining the size and shape dependence of gold nanoparticle uptake into mammalian cells". Nano Lett, 6(4): p. 6628 (2006).

[68] Steven D. Perrault and Warren C. W. Chan, "In vivo assembly of nanoparticle components to improve targeted cancer imaging" Proc Natl Acad Sci U S A.; 107(25): 11194-11199(2010).

[69] Chou C-Y, Huang C-K, Lu K-W, Horng T-L, Lin W-L Investigation of the Spatiotemporal Responses of Nanoparticles in Tumor Tissues with a Small-Scale Mathematical Model. PLoS ONE; 8(4): e59135(2013). 
[70] Barua, Sutapa, and Samir Mitragotri. "Challenges Associated with Penetration of Nanoparticles across Cell and Tissue Barriers: A Review of Current Status and Future Prospects." Nano today 9 (2) : 223-243(2014).

[71] Carletti, E.; Motta, A.; Migliaresi, C. "Scaffolds for tissue engineering and 3D cell culture". Methods Mol. Biol; 695, 17-39(2011).

[72] Friess W. "Collagen-biomaterial for drug delivery". Eur J Pharm Biopharm ;45: 113-36(1998).

[73] Kasoju, N., et al., "Exploiting the potential of Collagen as a Natural Biomaterial in Drug Delivery”. Journal of Proteins \& Proteomics, 1(1)(2013).

[74] Shoulders MD, Raines RT. "Collagen structure and stability". Annu Rev Biochem;78:929-958(2009).

[75] E.J. Kucharz, "The Collagens: Biochemistry and Pathophysiology”, Springer-Verlag, Berlin, pp. 7-29(1992).

[76] W. Friess, "Collagen: biomaterial for drug delivery", Eur. J. Pharm. Biopharm. 45 113-136(1998).

[77] Sell, S.A, Wolfe, P.S, Garg, K, McCool, J.M, Rodriguez, I.A.; Bowlin, G.L. "The Use of Natural Polymers in Tissue Engineering: A Focus on Electrospun Extracellular Matrix Analogues". Polymers; 2, 522-553(2010).

[78] Peralta L, Mourier E, Richard C, Charpigny G, Larcher T, Ait-Belkacem D, et al. "In vivo evaluation of cervical stiffness evolution during induced ripening using shear 
wave elastography, histology and 2 photon excitation microscopy: insight from an animal model”. PLoS One;10(8):e0133377(2015).

[79] Wang MH, Palmeri ML, Guy CD, Yang L, Hedlund LW, Diehl AM, Nightingale KR. "In vivo quantification of liver stiffness in a rat model of hepatic fibrosis with acoustic radiation force". Ultrasound Med Biol ;35:1709-1721(2009).

[80] Blackmon, Richard L., Rupninder Sandhu, Brian S. Chapman, Patricia CasbasHernandez, Joseph B. Tracy, Melissa A. Troester, and Amy L. Oldenburg. "Imaging Extracellular Matrix Remodeling In Vitro by Diffusion-Sensitive Optical Coherence Tomography." Biophysical journal 110(8): 1858-1868(2016).

[81] Y. Ponce de León, J. L. Pichardo-Molina, N. Alcalá Ochoa, and D. Luna-Moreno, "Contrast Enhancement of Optical Coherence Tomography Images Using Branched Gold Nanoparticles," Journal of Nanomaterials, 2012,128,(2012).

[82] Braz, Ana KS, Renato E. de Araujo, Tymish Y. Ohulchanskyy, Shoba Shukla, Earl J. Bergey, Anderson SL Gomes, and Paras N. Prasad. "In situ gold nanoparticles formation: contrast agent for dental optical coherence tomography." Journal of biomedical optics ,17(6): 066003-066003(2012).

[83] Zagaynova, E.V., Shirmanova, M.V., Kirillin, M.Y., Khlebtsov, B.N., Orlova, A.G., Balalaeva, I.V., Sirotkina, M.A., Bugrova, M.L , Agrba, P.D. and Kamensky, V.A., 2008. Contrasting properties of gold nanoparticles for optical coherence tomography: phantom, in vivo studies and Monte Carlo simulation.Physics in Medicine and Biology, 53(18), p.4995. 
[84] S. H. Yun, G. J. Tearney, J. F. de Boer, and B. E. Bouma, "Motion artifacts in optical coherence tomography with frequency-domain ranging," Opt. Express,12(13),29772998(2004).

[85] A. Mariampillai, B. A. Standish, N. R. Munce, C. Randall, G. Liu, J. Y. Jiang, A. E. Cable, I. A. Vitkin, and V. Yang, "Doppler optical cardiogram gated 2D color flow imaging at $1000 \mathrm{fps}$ and 4D in vivo visualization of embryonic heart at $45 \mathrm{fps}$ on a swept source ," Opt. Express, 15( 4), 1627-1638 (2007).

[86] Li, Xiang, et al. "Integrated IVUS-OCT catheter for in vivo intravascular imaging." Ultrasonics Symposium (IUS), 2012 IEEE International. IEEE, (2012).

[87] Li B.H., Leung A.S., Soong A., Hybrid intravascular ultrasound and optical coherence tomography catheter for imaging of coronary atherosclerosis. Catheter Cardiovasc Interv.;81:494-507(2013).

[88] Wei-Cheng Kuo, Jongsik Kim, Nathan D. Shemonski, Eric J. Chaney, Darold R. Spillman, Jr., Stephen A. Boppart "Real-time three-dimensional optical coherence tomography image-guided core-needle biopsy system ," Biomed Opt Express.; 3(6): 1149-1161(2012).

[89] Hao-Chung Yang, Jiechen Yin, Changhong Hu, Jonathan Cannata, Qifa Zhou, Jun Zhang, Zhongping Chen, K. Kirk Shung “A Dual-Modality Probe Utilizing Intravascular Ultrasound and Optical Coherence Tomography for Intravascular Imaging Applications",IEEE Trans Ultrason Ferroelectr Freq Control. 57(12): 2839-2843(2010). 
[90] Oldenburg AL, Crecea V, Rinne SA, Boppart SA. "Phase-resolved magnetomotive OCT for imaging nanomolar concentrations of magnetic nanoparticles in tissues”. Optics express ;16(15):11525-11539(2008).

[91] Nguyen T-M, Song S, Arnal B, Huang Z, O’Donnell M, Wang RK. "Visualizing ultrasonically-induced shear wave propagation using phase-sensitive optical coherence tomography for dynamic elastography”. Optics letters.;39(4):838-841(2014). 


\section{Glossary}

\begin{tabular}{|l|l|}
\hline ARF & Acoustic Radiation Force \\
\hline SS-OCT & Swept Source Optical Coherence Tomography \\
\hline SV & Speckle Variance \\
\hline NP & Nanoparticle \\
\hline OCT & Optical Coherence Tomography \\
\hline OCE & Optical Coherence Elastography \\
\hline US & Ultrasound \\
\hline GNPs & Gold nanoparticles \\
\hline SD-OCT & Spectral Domain OCT \\
\hline SW & Shear Wave \\
\hline MR & Magnetic Resonance \\
\hline NA & Numerical Aperture \\
\hline HIFU & High Frequency Ultrasound \\
\hline S-OCE & Spectroscopic OCE \\
\hline FUS & Focused Ultrasound \\
\hline ROI & Region of interest \\
\hline FEM & Finite element methods \\
\hline MRI-FUS & MRI-guided focused ultrasound \\
\hline MRIFUS & MRI-guided focused \\
\hline
\end{tabular}

